$1 /\left.3\right|^{23} /\left.\left.\right|^{16}\right|_{\text {Thesis }} ^{9}$

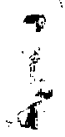

\section{MR. 2344}

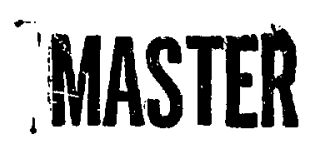

\title{
Analysis of Error in Monte Carlo Transport Calculations
}


LA-7636-T

Thesis

UC.34c

Issued January 1979

\section{Analysis of Error in}

\section{Monice Carlo Transport Calculations}

Thomas Edward Booth

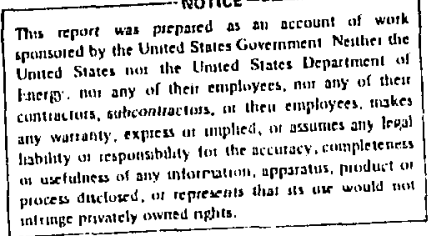

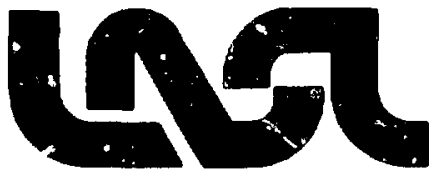

n:

8 
TABLE OF CONTENTS

INTRODUCTION ................................... vit

CHAPTER I Illustration of Concepts by Example ....... 1

IA Analog Game................ 2

IB Non-Analog Games ............ 10

IB-1 Biased Kernels. .............. 12

IB-2 Splitting, Russian Roulette, and Weight Cutoff. . 19

IC Remarks on the Relationship of Chapter I to Chapter III. ......... 20

Chapter II Definitiois ............... 21

Chapter III Derivation of Moment Equations and Choice of Weights ........... 28

IIIA Derivation ................ 28

IIIB Choice of Non-Analog Kernels and Weight Multipliers........... 45

Chapter IV Examples................ 58

IVA Collision and Track Length Estimators Compared. . 58

IVB One-Speed Infinite Medium Equations ....... 66

IVC Theoretical Comparison of Four Estimators for Collisions............. 71

IVD Numerical Comparison of Four Estimators for Collisions. ............. 88

Chapter $v$ Conclusion. ............... 93 


\section{APPENDICES}

Appendix A Useful Results ............... 95

Appendix B Simple Example of an Unreliable

Sample Variance. . . . . . . . . . . 99

Appendix C Convergence of the Neumann Series

for Equation (125)............ 100

Appendix D Approximation of a Bounded $n$-Dimensional

Region by Convex Subregions. . . . . . . 107

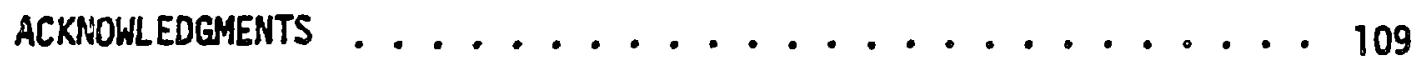

REFERENCES .......................... 110 


\section{INTRODUCTION}

The Monte Carlo method attempts to get the average behavior of neutrons by simulating large sample of neutron histories and using the sample mean as an approximation ${ }^{+}$to the average predicted by the Neutron Transport Equation. The Monte Carlo method for neutron transport calculations suffers, in part, because of the inherent statistical errors associated with the method. Without an estimate of these errors in advance of the calculation, it is difficult to decide what estimator and biasing scheme to use, or whether a proposed Monte Carlo calculation will be competitive with a discrete ordinates calculation (a question not treated here).

Perhaps the most compelling reason for predicting statistical errors is cost. If one is contemplating writing a Monte Carlo code (or making major modifications to an existing code) incorporating a rew Monte Carlo technique, he would like to know how well the new technique will work - before investing a great deal of time and money writing the code.

If one uses a standard Monte Cario code he would probably try several techniques and uses the sample variance to select the best technique. This trial and error method works well if one has enough insight into both the transport ,roblem and the Monte Carlo techniques he applies to solve it. However, if one's insight is poor, trial and error will result Using the law of large numbers 
in mostly error. Furthermore, the sample variance, by which one judges the technique, becomes more and more unreliable as the technique departs from optimum. Afpendix $B$ has an example of an unreliable sample variance.

Recent work $2,3,4,7,8$ on the prediction of statistical errors in Monte Carlo caiculations stems from a paper Amster and D.jomehri ${ }^{1}$ pub1ished in 1976, in which a set of integral equations were derived that, when solved, predicted errors in Monte Carlo calculations in non-multiplying media. The present work allows error prediction in non-analog Monte Carlo calculations of multiplying systems, even when supercritical. Non-analog techniques such as biased kernels, particle splitting, and Russian Roulette have been incorporated in this work. Coincidentally (and independently of this work), Sarkar and Prasad ${ }^{3}$ have investigated errors in non-analog Monte Carlo calculations of subcritical multiplying systems. 


\section{Chapter 1. Illustration of Concepts by Example}

The Monte Carlo method simulates neutron behavior on a computer. Real neutron transport problems are quite complex and the mathematics required to predict errors in their Monte Carlo simulations is correspondingly complex. In fact, the mathematics of ten obscures some very simple corcepts. This chapter illustrates, for a very simple transport problem, concepts such as analog game, non-analog game, particle weight, score, and importance sampling - using only high-school mathematics. The reader who is familiar with Monte Carlo may skip to Chapier II which treats real transport problems.

An analog (Monte Carlo) game is a faithful simulation of the real transport process; a non-analog game is a simulation in which the simulated neutron does not behave exactly like a real neutron. As a simple example, a two-state transport problem is simulated - first by an anaiog game in I-A and then by a non-analog game in I-B. Section I-C links the analysis of this simple problem to the analysis of the real transport problems considered in Chapters II and III.

A few definitions are required before addressing the two-state transport problem. In subsequent discussions, particle refers to a computer simulated neutron. A particle's history is a sequence of events, chosen in a random fashion, ending with the loss of the particle. Every event (e.g. scattering) has two numbers associated with it: the probability the event occurs and the event score. A particle's history 
score (total score) is the sum of all the event scores the particle generates in its history. Although nature supplies event probabilities, at least for analog games, the calculator must assign appropriate event scores. For example, if one is interested in the nean number of scattering events per particle he might assign each scattering event a "one" and any other event a "zero"; a particle's history score would then be equal to the number of times the particle was scattered.

\section{A) Analog Game}

Consider the simple transport problem below where a particle can be in one of two states, labeled 1 and 2. The six possible events and their probabilities and scores are depicted in figure 1 and described in table 1. These six eyents are independent and mutualiy exclusive.

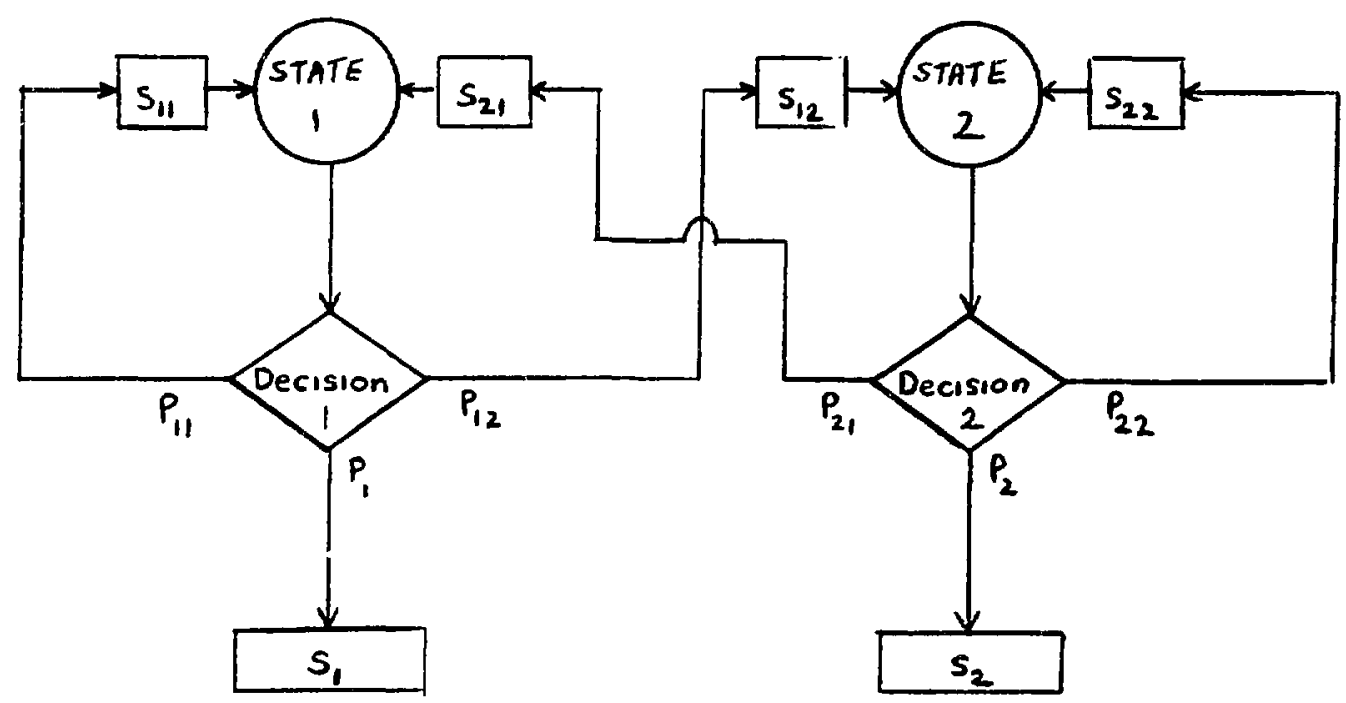

Fig 1. The two-state transport problem. 


\section{Tabie I}

Definitions for the two-state transport problem

\begin{tabular}{|c|c|c|}
\hline Event & $\begin{array}{c}\text { Event } \\
\text { Probability } \\
\end{array}$ & Event Score \\
\hline${ }_{12}$ scattering from state 1 to state 2 & $P_{12}$ & $s_{12}$ \\
\hline$e_{21}$ scattering from state 2 to state 1 & $P_{21}$ & $s_{21}$ \\
\hline$e_{11}$ scattering from state $l$ to state 1 & $p_{11}$ & $s_{11}$ \\
\hline$e_{22}$ scattering from state 2 to state 2 & $P_{22}$ & $s_{22}$ \\
\hline${ }_{1}$ absorption from state 7 & $P_{1}$ & $s_{1}$ \\
\hline$e_{2}$ absorption from state $?$ & $P_{2}$ & $s_{2}$ \\
\hline
\end{tabular}


Decision 1 will be made by picking a random number, $\zeta_{i}$, between 0 and 1:

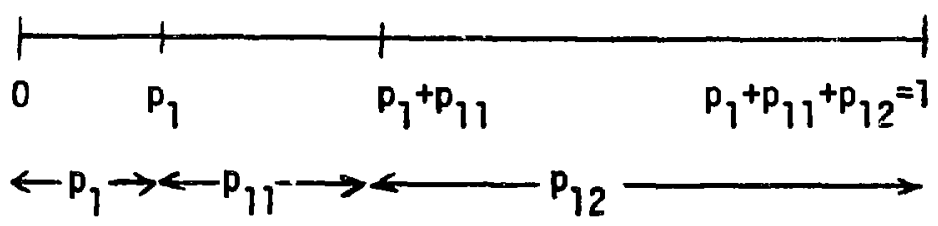

$e_{i}$ occurs if $0<\zeta_{j} \leq p_{i}$,

$e_{11}$ occurs if $p_{1}<\zeta_{j}<p_{1}+p_{11}$, and

$e_{12}$ occurs if $p_{1}+p_{11}<\zeta_{j} \leq l$.

Decision 2 will be made by picking a random number, $\zeta_{j}$, between 0 and 1 :

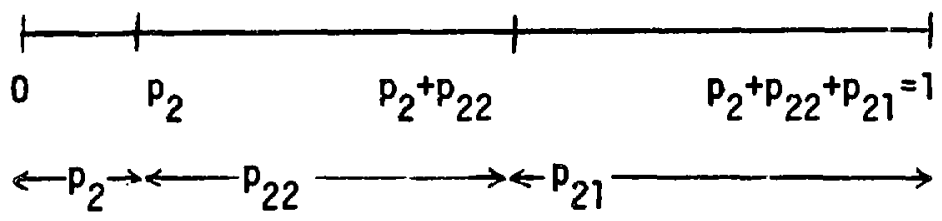

$e_{2}$ occurs if $0<\zeta_{i} \leq p_{2}$,

$\epsilon_{22}$ occurs if $p_{2}<\zeta_{i}<p_{2}+p_{22}$, and

$e_{21}$ occurs if $p_{2}+p_{22}<\zeta_{i} \leq 1$.

As an example, consider a particle starting in state 1. Suppose that the $\zeta_{j}$ are:

$$
\begin{aligned}
& p_{1}+p_{11}<\zeta_{1} \leq 1 \text { implies } e_{12} \text { occurs, } \\
& p_{2}<\zeta_{2} \leq p_{2}+p_{22} \text { implies } e_{22} \text { occurs: } \\
& p_{2}+p_{22} \leq \zeta_{3}<i \quad \text { implies } e_{21} \text { occurs, and } \\
& 0<\zeta_{4} \leq p_{1} \quad \text { implies } e_{1} \text { occurs. }
\end{aligned}
$$


In this case, referring to Tabie 1 , the particle first scatters from state 1 to state 2 (event $e_{12}$ ) and scores $s_{12}$, next scatters from state 2 to state 2 (event $e_{22}$ ) and scores $s_{22}$, next scatters from state 2 to state 1 (event $e_{21}$ ) and scores $s_{21}$, and lastly suffers absorption from staie 1 (event $e_{1}$ ) and scores $s_{1}$. The history score for this series of events is then $s=s_{12}+s_{22}+s_{21}+s_{1}$.

This simple system could represent a particle roaming about in an infinite homogeneous two nuclide medium (with only scattering and absorption)

State $1=$ particle entering a collision with nuclide type 1

State 2 = particls entering a collision with nuclide type 2 If the scores are taken to be

$$
s_{11}=s_{12}=s_{21}=s_{22}=s_{2}=0 \text { and } s_{1}=1 \text {, }
$$

then a particle absorbed by nuclide 1 would have a history score of 1 and a particle absorbed by nuclide 2 would have a history score of 0 . If a large number of particles starting at nuclide $j$ are followed, the mean history score is an estimate of the inean number of particies absorbed by nuclide 1 per particle entering a collision with nuclide $j$. (This problem is easily solved without Monte Carlo.)

Now return to the general two state problem. It is important to know how many particles must be followed to obcain an acceptable error in the estimate of the mean history score. Let $M_{1}$ be the mean score and $M_{2}$ be the mean-square score. According to the "central limit theorem ${ }_{2} 1$ the means of samples, each of $h$ histories, for $h$ large, 
are approximately normally distributed about $M_{1}$ with a variance

$$
\sigma^{2}=\frac{M_{2}-M_{1}^{2}}{h} .
$$

Ai? estimate of this variance can be obtained by substituting the sample means $\overline{\bar{m}}_{1}$ and $\bar{M}_{2}$ in place of the means $M_{1}$ and $M_{2}$. There are two problems with using the sample variance:

1) The error estimate is available only after the problem has been programmed and run on a computer. Thus much investment in programming time and computer time might be required simply to determine that the variance is unacceptably high.

2) The sample means $\bar{M}_{1}$ and $\bar{M}_{2}$ tend to be unreliable estimates of $M_{1}$ and $M_{2}$ when the variance is high. Suppose $\bar{M}_{2}<M_{2}$ and $M_{1}^{2}>\bar{M}_{1}^{2}$; this results in a sample variance,

$$
\bar{\sigma}^{2}=\frac{\bar{M}_{2}-\bar{M}_{1}^{2}}{h}<\frac{M_{2}-M_{1}^{2}}{h}=\sigma^{2} \text {, }
$$

which could be much less than the true variance.

As an example of the second problem, consider the two nuclide problem with the absorption cross section for nuclide 1 many orders of magnitude smaller than any other cross sections. This corresponds to $p_{1}$ being orders of magnitude smaller than any of the other probabilities. Thus 100,000 particles might be followed and all result in absorption by nuclide 2, i.e. history score zero. In this case,

$$
\begin{aligned}
& \bar{M}_{1}=0 \text {, sample mean history score, } \\
& \bar{M}_{2}=0 \text {, sample mean-square history score, and }
\end{aligned}
$$




$$
\bar{\sigma}^{2}=\frac{\bar{M}_{2}-\bar{M}_{1}^{2}}{h}=0, \text { sample variance, }
$$

whereas, in fact, $M_{1}=\varepsilon$, where $\varepsilon$ is some number greater than zero. Thus, $\bar{M}_{1}$ is infinitely smaller than the true mean $M_{1}$ at the same time that the sample variance of zero indicates a very good sample mean.

To avoid problems one and two it is useful to obtain an estimate of the variance before attempting a Monte Carlo solution of the problem. To obtain this estimate three terms must be defined:

$$
\begin{aligned}
\psi(j, s)= & \text { probability that a particle in state } j \text { will contribute } \\
& \text { a cumulative score } s \text { in its subsequent history; }
\end{aligned}
$$

$$
\begin{aligned}
& M_{r}(j)=\sum_{s} s^{r} \psi(j, s)=r^{\text {th }} \text { moment of history score } \\
& \text { (where the sum is over all possible } \\
& s \text { values); and } \\
& \delta_{s s^{\prime}}=\left\{\begin{array}{l}
0 \text { if } s \neq s^{\prime}, \\
1 \text { if } s=s^{\prime},
\end{array}\right.
\end{aligned}
$$

From these definitions eqn. (7) may be derived.

$$
\psi(i, s)=p_{i} \delta_{s s_{i}}+\sum_{j} p_{i j} \psi\left(j, s-s_{i j}\right)
$$

Let $s_{n e}\left(s_{n e} \varepsilon\left\{s_{1}, s_{17}, s_{12}, s_{2}, s_{22}, s_{21}\right\}\right)$ be the score contributed by the next event. The probability that a particle in state $i$ subsequently contributes a cumulative score $s$ is equal to the sum, over all possible 
next events, of the probability that the given event occurs multiplied by the probability that all subsequent events contribute the remaining score $s-s_{n e}$, so that the total score is $s_{n e}+\left(s-s_{n e}\right)=s$. If the next event is an absorption then the probability that the cumulative score is $s$ is $\delta_{s s_{i}}$; that is, if the absorption contributes a score $s$ the cumulative score will be $s$ and if the absorption does not contribute a score $s$ the cumulative score will not be $s$. If the next event is a scattering to state $j$ then there is a score $s_{i j}$ contributed by the scattering, which leaves a score s-s ${ }_{i j}$ to be contributed in subsequent events. The probability that a particle in state $j$ contributes a cumulative score $s-s_{i j}$ in subsequent events is $\psi\left(j, s-s_{i j}\right)$ by definition (4).

Multiplying eq. (7) by $s^{r}$ and summing over all possible scores,

$$
\sum_{s} \psi(i, s) s^{r}=\sum_{s} \delta s s_{i} p_{i} s^{r}+\sum_{s} \sum_{j} p_{i j} \psi\left(j, s-s_{i j}\right) s^{r} .
$$

Using eq. (5) and recalling that $s_{j}$ is a possible value for $s$,

$$
M_{r}(i)=p_{i} s_{i}^{r}+\sum_{s} \sum_{j} p_{i j} \psi\left(j, s-s_{i j}\right) s^{r}
$$

Letting $s^{\prime}=s-s_{i j}$ or $s=s^{\prime}+s_{i j}$

$$
\sum_{s} \sum_{j} p_{i j} \psi\left(j, s-s_{i j}\right) s^{r}=\sum_{s^{\prime}} \sum_{j} p_{i j} \psi\left(j, s^{\prime}\right)\left(s^{\prime}+s_{i j}\right)^{r} ;
$$

then using the binomial expansion,

$$
=\sum_{s^{\prime}} \sum_{j} p_{i j} \psi\left(j, s^{\prime}\right) \sum_{n=0}^{r}\left(\begin{array}{l}
r \\
n
\end{array}\right) s^{i n} s_{i j}^{r-n}
$$




$$
=\sum_{j} p_{i j} \sum_{n=0}^{r}\left(\begin{array}{l}
r \\
n
\end{array}\right) s_{i j}^{r-n} \quad \sum_{s^{i}} \psi\left(j, s^{\prime}\right) s^{\prime n},
$$

and by using eq. (5)

$$
=\sum_{j} p_{i j} \sum_{n=0}^{r}\left(\begin{array}{l}
r \\
n
\end{array} s_{i j}^{r-n} M_{n}(j)\right.
$$

Substituting eq. (10) into eq. (9) yields the results,

$$
M_{r}(i)=p_{i} s_{i}^{r}+\sum_{j} p_{i j} \sum_{n=0}^{r}\left(\begin{array}{l}
r \\
n
\end{array}\right) s_{i j}^{r-n} M_{n}(j) .
$$

Pulling out the $M_{r}$ term from the sum,

$M_{r}(j)=\sum_{j} p_{i j} M_{r}(j)+p_{i} s_{i}^{r}+\sum_{j} p_{i j} \sum_{n=0}^{r-1}\left(\begin{array}{l}r \\ )\end{array} s_{i j}^{r-n} M_{n}(j)\right.$

and noting that

$$
M_{0}(j)=\sum_{s} \psi(j, s)=1
$$

since $\psi(j, s)$ is a probability function, eq. (12) for $r=1$ and $r=2$ may be written:

$$
\begin{aligned}
& M_{1}(1)=p_{11} M_{1}(1)+p_{12} M_{1}(2)+p_{1} s_{1}+p_{11} s_{11}+p_{12} s_{12} \\
& M_{1}(2)=p_{21} M_{1}(1)+p_{22} M_{1}(2)+p_{2} s_{2}+p_{22} s_{22}+p_{21} s_{21}
\end{aligned}
$$

and

$$
\begin{aligned}
M_{2}(1) & =p_{11} M_{2}(1)+p_{12} M_{2}(2)+p_{1} s_{1}^{2}+p_{11}\left(s_{11}^{2}+2 s_{11} p_{1}(1)\right) \\
& +p_{12}\left(s_{12}^{2}+2 s_{12} M_{1}(2)\right)
\end{aligned}
$$




$$
\begin{aligned}
M_{2}(2) & =p_{22} M_{2}(2)+p_{21} M_{2}(1)+p_{2} s_{2}^{2}+p_{22}\left(s_{22}^{2}+2 s_{22} M_{1}(2)\right) \\
& +p_{21}\left(s_{21}^{2}+2 s_{21} M_{1}(1)\right)
\end{aligned}
$$

IB Non-Ana log Games

The equations derived above are for an analog simulation of the real transport process; the probability that event $i$ cccurs in the simulation is the same as the probability that event $i$ occurs in the real transport process. In practice, the transport problem can sometimes be solved more accurately, for a given number of particle histories, if fictitious probabilities are used instead of the real transport probabilities. Any Monte Carlo soiution which is not an analog simulation of the real transport process is termed a non-arialog Monte Carlo solution.

To understand non-analog Monte Carlo it is necessary to introduce the concept of statistical weight for a particle. Statistical weight, or simply weight, is a score multiplier. Thus, for a given event a particle of weight $w$ scores $w$ times as much as a particle of weight one.

Consider the general two state problem with the particle in state j. There are three chainis of events which may occur in the analog game considered previously:

1) the particle is absorbed in state $j$ contributing a cuniulative history score $s_{a}=s_{j}$ 
2) the particle scatters to state 1 and contributes a score $s_{j l}$. Subsequently, from state 1 the particle contributes a score $s_{b}-s_{j l}$ resulting in a cumulative history score from state $j$ of $s_{j l}+\left(s_{b}-s_{j l}\right)=s_{b}$

3) the particle scatters to state 2 and contributes a score $s_{j 2}$. Subsequently, from state 2 the particle contributes a score $s_{c}-s_{j 2}$ resulting in a cumulative history score from state $j$ of $s_{j 2}+\left(s_{c}-s_{j 2}\right)=s_{c}$.

The maan score for a particle in state $j$ is then

$$
\begin{aligned}
& \left.M_{1}(j)=\sum_{i=1}^{3} \text { (prob chain } i \text { occurs }\right) \star(\text { score due to chain } i) \\
& M_{l}(j)=p_{j} s_{a}+p_{j 1} s_{b}+p_{j 2} s_{c} .
\end{aligned}
$$

Suppose that the probabilities are altered in the following manner,

$$
\tilde{p}_{j}=\frac{l}{\alpha} p_{j}, \quad \tilde{p}_{j 1}=\frac{l}{B} p_{j 1} \text {, and } \quad \tilde{p}_{j 2}=\frac{l}{\gamma} p_{j 2}
$$

subject to the consiraint that the probability that one of the three events occurs is still one:

$$
\tilde{p}_{j}+\tilde{p}_{j 1}+\tilde{p}_{j 2}=1 \text {. }
$$

Then substituting eq. (20) into eq. (19),

$$
M_{1}(j)=\alpha \tilde{p}_{j} s_{a}+\beta \tilde{p}_{j 1} s_{b}+\tilde{p}_{j 2} s_{c} \cdot
$$

Recall that for the same chain of events a particle of weight $w$ contributes $w$ tines the score that a particle of weight one contributes. 
Identifying $\alpha=w_{7}, \beta=w_{2}$, and $\gamma=w_{3}$ as particle weights eq. (18) can be generalized to:

$M_{1}(j)=\sum_{i=1}^{3}(\text { prob chain } i \text { occurs in caic })^{\star}($ score due to chain $i$
from a particle of weight $\left.w_{i}\right)$.

While the mean score, $M_{1}$, remains the same with the fictitious probiabilities the mean square score in general does not. To put these ideas on a more rigorous footing eqs. $(14)-(17)$ will be rederived for arbitrary branch probabilities and branch weight multipliers. When the branch probabilities are not the same as the real transport probabilities this is called biasing or importance sampling.

$\underline{\text { IB-1 Biased Kerne?s }}$

Consider a completely fictitious game in which the probabilities, weights, and scores are completely arbitrary; later it will be shown that with suitable choices for probabilities, weights, and scores, this non-analog game can be made to yield the same mean score, $M_{1}$, as the analog game. The following definitions are needed:

$\tilde{\psi}(j, w, s)=$ probability that a particle of weight $w$ in state $j$ contributes a score $s$,

$w_{i}=$ weight multiplier for absorption from state $i$

$w_{i j}=$ weight multiplier for scattering from state $i$ to state $j$, 


$$
\begin{aligned}
& \tilde{p}_{j}=\text { prob of absorption from state } i, \\
& \tilde{p}_{i j}=\text { prob of scattering froli state } i \text { to state } j, \\
& \tilde{M}_{r}(j, w)=\sum_{s} s^{r} \tilde{\psi}(j, w, s)=r^{\text {th }} \text { moment of history score for a } \\
& \text { particle of weight } w \text { in state } j,
\end{aligned}
$$

$$
\begin{aligned}
\tilde{s}_{i}(w)= & \text { score for absorption from state } i \text { of a particle of } \\
& \text { weight } w
\end{aligned}
$$

$$
\tilde{s}_{i j}(w)=\text { score for scattering from state } i \text { to state } j \text { for } a
$$
particle of weight $w$, and

$$
\delta_{s s^{\prime}}=\left\{\begin{array}{l}
0 \text { if } s^{\prime} \neq s^{\prime} \\
1 \text { if } s=s^{\prime}
\end{array} \text { where } s \text { and } s^{\prime}\right. \text { are not necessarily integers. }
$$

Next, consider eqn. (3j) which is displayed and explained below.

$$
\tilde{\psi}(i, w, s)=\tilde{p}_{i} \delta_{s s_{i}}\left(w w_{i}\right)+\sum_{j} \tilde{p}_{i j} \tilde{\psi}\left(j, w w_{i j}, s-\tilde{s}_{i j}\left(w w_{i j}\right)\right)
$$

Let $s_{n e}$ be the score contributed by the next event. The probability that a particle of weight $w$ in state $i$ subsequently contributes a cumulative score $s$ is equal to the sum, over all possible next events, of the probability that the given event occurs multiplied by the probability that all subsequent events contribute the remaining score $s-s_{n e}$, so that the total score is $s_{n e}+\left(s-s_{n e}\right)=s$.

If the next event is absorption then the particle's weight becomes $w_{i}$ and the probability that the cumulative score is $s$ is 
$\delta_{s \tilde{s}_{i}}\left(w w_{i}\right)$ That is, the cumulative score will be $s$ if and only if the absorption contributes a score s.

If the next event is a scattering to state $j$ then the particle's weight becomes $w w_{i j}$ and there is a score $\tilde{s}_{i j}\left(w w_{i j}\right)$ contributed by the scattering. This leaves a score $s-\tilde{s}_{i j}\left(W w_{i j}\right)$ to be contributed in subsequent events. The probability that a particle of weight $\mathrm{ww}_{i j}$ in state $j$ contributes a cumulative score $s-\tilde{s}_{i j}\left(w w_{i j}\right)$ in subsequent events is by definition $\psi\left(j, w w_{i j}, s-\tilde{s}_{i j}\left(w w_{i j}\right)\right)$.

Multiplying eqn. (33) by $s^{r}$ and summing over all possible values for s yields:

$$
\begin{gathered}
\sum_{s} \tilde{\psi}(i, w, s) s^{r}=\sum_{s} \tilde{p}_{i} \delta_{s \tilde{s}_{j}\left(w w_{j}\right)^{s}}+\sum_{s} \sum_{j} \tilde{p}_{i j} \\
\times \tilde{\psi}\left(j, w w_{i j}, s-\tilde{s}_{i j}(w w, j)\right) s^{r} .
\end{gathered}
$$

Using the definition of $\tilde{M}_{r}$ and recalling that $\tilde{s}_{j}\left(w_{j}\right)$ is a possible value for $s$,

$$
\begin{aligned}
& \tilde{M}_{r}(i, w)=\tilde{p}_{i} \tilde{s}_{i}^{r}\left(w w_{i}\right)+\sum_{s} \sum_{j} \tilde{p}_{i j} \tilde{\psi}\left(j, w w w_{i j}, s-\tilde{s}_{i j}(w w i j)\right) s^{r} . \\
& \text { Letting } s^{\prime}=s-\tilde{s}_{i j}\left(w w_{i j}\right) \text { or } s=s^{\prime}+\tilde{s}_{i j}\left(w w_{i j}\right) \text { yields } \\
& \sum_{s} \sum_{j} \tilde{p}_{i j} \tilde{\psi}\left(j, w w_{i j}, s-\tilde{s}_{i j}\left(w w_{i j}\right)\right) s^{r} \\
& =\sum_{s^{\prime}} \sum_{j} \tilde{p}_{i j} \tilde{\psi}\left(j, w w_{i j}, s^{\prime}\right)\left(s^{\prime}+\tilde{s}_{i j}\left(w w_{i j}\right)\right)^{r},
\end{aligned}
$$

and using the binomial expansion, 


$$
\begin{aligned}
& =\sum_{s} \sum_{j} \tilde{p}_{i j} \tilde{\psi}\left(i, w w w_{i j}, s^{\prime}\right) \sum_{n=0}^{r}\left(l_{n}^{r}\right) s^{\prime n}\left[\tilde{s}_{i j}\left(k, w_{i j}\right)\right]^{j-n} \\
& =\sum_{j} \tilde{p}_{i j} \sum_{n=0}^{r}\left(r_{n}^{r}\right)\left[\tilde{s}_{i j}\left(w w_{i j}\right)\right]^{r-n} \sum_{s^{\prime}} \tilde{\psi}\left(j, w w_{i j}, s^{\prime}\right) s^{\prime n} .
\end{aligned}
$$

From the definition of $\tilde{M}_{r}$,

$=\sum_{j} \tilde{p}_{i j} \sum_{n=0}^{r}\left(r_{n}^{r}\right)\left[\tilde{s}_{i j}\left(w w w_{i j}\right)\right]^{r-n} \tilde{m}_{n}\left(j, w w w_{i j}\right)$

hence,

$\tilde{M}_{r}(i, w)=\tilde{p}_{i} \tilde{s}_{i}^{r}\left(w w_{i}\right)+\sum_{j} \tilde{p}_{i j} \sum_{n=0}^{r}\left(r_{n}^{r}\right)\left[\tilde{s}_{i j}\left(w w w_{i j}\right)\right]^{r-n} \tilde{M}_{n}\left(j, w w w_{i j}\right)$,

and pulling out the $M_{r}$ term from the sum,

$$
\begin{aligned}
\tilde{M}_{r}(i, w) & =\sum_{j} \tilde{p}_{i j} \tilde{M}_{r}\left(j, w w_{i j}\right)+\tilde{p}_{i} \tilde{s}_{i}^{r}\left(w w_{i}\right) \\
& +\sum_{j} \tilde{p}_{i j} \sum_{n=0}^{r-1}\left(\begin{array}{l}
r \\
n
\end{array}\right)\left[\tilde{s}_{i j}\left(w w_{i j}\right)\right]^{r-n} \tilde{M}_{n}\left(j, w w_{i j}\right) .
\end{aligned}
$$

Since for a given event a particle of weight $w$ scores $w$ times as much as a particle of weight 1 ,

$$
\begin{aligned}
& \tilde{s}_{i}\left(w w_{i}\right)=w w_{i} \tilde{s}_{i}(1) \\
& \tilde{s}_{i j}\left(w w_{i j}\right)=w w_{i j} \tilde{s}_{i}(1) .
\end{aligned}
$$

Writing eqn. (39) for $r=1$ (Noting as in eqn. (13)

$$
\begin{gathered}
\left.\tilde{M}_{0}(j, w)=\sum_{s} \tilde{\psi}(j, w, s)=1\right), \\
\tilde{M}_{1}(i, w)=\sum_{j} \tilde{p}_{i j} \tilde{M}_{1}\left(j, w w_{i j}\right)+\tilde{p}_{j} w w_{i} \tilde{s}_{j}(1)+\sum_{j} \tilde{p}_{i j} w w_{i j} \tilde{s}_{i j}(1) .
\end{gathered}
$$


A particle's history score is proportional to its weight because:

1) the event probabilities are assumed independent of weight; thus

2) a particle's history (sequence of events) is independent of weight, and

3) each event score is proportional to the particle's weight.

Thus the probability that a particle of weight $w$ contributes a history score $s$ is equal to the probability that a particle of weight one contributes a history score $s / w$, that is

$\Psi(i, w, s)=\Psi(i, 1, s / w)$

Hence using eqs. (29) and (43A)

$\tilde{M}_{r}(i, w)=\sum_{s} \Psi(i, w, s) s^{r}=w^{r} \sum_{s} \Psi(i, 1, s / w)(s / w)^{r}=w^{r} \tilde{M}_{r}(i, 1)$

Thus eq. (43) becomes

$$
\tilde{M}_{1}(i, 1)=\sum_{j} \tilde{p}_{i j} w_{i j} \tilde{M}_{1}(j, 1)+\tilde{p}_{i} w_{i} \tilde{s}_{i}(1)+\sum_{j} \tilde{p}_{i j} w_{i j} \tilde{s}_{i j}(1) .
$$

Suppose the probabilities, weights, and scores are chosen such that (remember they were arbitrary):

$\tilde{p}_{i j} w_{i j}=p_{i j} \quad \tilde{p}_{i} w_{i}=p_{i} \quad \tilde{s}_{i}(1)=s_{i} \quad \tilde{s}_{i j}(1)=s_{i j}$

then,

$$
\tilde{M}_{1}(j, 1)=\sum_{j} p_{i j} \tilde{M}_{1}(j, 1)+p_{j} s_{i}+\sum_{j} p_{i j} s_{i j}
$$


This is the same eqn. that $M_{1}(i)$ satisfies [cf. eq. (12)], hence

$$
\tilde{M}_{1}(i, i)=M_{1}(i) \text {. }
$$

The significance of this result is that a non-analog transport game (with relations (46) holding) can be played with any probabilities $\tilde{p}_{i j}>0\left(\tilde{p}_{i j}\right.$ must be greater than zero so that $w_{i j}$ is finite) and the mean score for the non-analog game will be equal to the mean score for the analog game. The second moments for the two games are in general not equal, as will be shown below.

Writing eq. (39) for $r=2$,

$$
\begin{aligned}
\tilde{M}_{2}(i, w) & =\sum_{j} \tilde{p}_{i j} \tilde{M}_{2}\left(j, w w_{i j}\right)+\tilde{p}_{i}\left[w w_{i} \tilde{s}_{i}(1)\right]^{2} \\
& +\sum_{j} \tilde{p}_{i j}\left(\left[w w_{i j} \tilde{s}_{i j}(1)\right]^{2}+2 w w_{i j} \tilde{s}_{i j}(1) w w_{i j} \tilde{M}_{1}(j, 1)\right) .
\end{aligned}
$$

From eq. (44) with $r=2$,

$$
\tilde{m}_{2}(i, w)=w^{2} \tilde{m}_{2}(i, i)
$$


Letting $w=1$ and using eq. (50)

$$
\begin{aligned}
\tilde{M}_{2}(i, 1) & =\sum_{j} \tilde{p}_{i j} w_{i j}^{2} \tilde{M}_{2}(j, 1)+\tilde{p}_{i}\left[w_{i} \tilde{s}_{i}(1)\right]^{2} \\
& +\sum_{j} \tilde{p}_{i j}\left(\left[w_{i j} \tilde{s}_{i j}(1)\right]^{2}+2 w_{i j}^{2} \tilde{s}_{i j} \tilde{M}_{1}(j, 1)\right) .
\end{aligned}
$$

Using the relations $(46)$ which resulted in $\tilde{M}_{p}(i, 1)=M_{p}(i)$,

$$
\begin{aligned}
\tilde{M}_{2}(i, l) & =\sum_{j} p_{i j} w_{i j} \tilde{M}_{2}(j, 1)+p_{i} w_{i} s_{i}{ }^{2} \\
& +\sum_{j} p_{i j}\left(w_{i j} s_{i j}^{2}+2 w_{i j} s_{i j} \tilde{M}_{1}(j)\right) .
\end{aligned}
$$

This is not the same as eqns. (16) and (17), hence

$$
\tilde{M}_{2}(i, 1) \neq M_{2}(i)
$$

It has been shown that there is a class of non-analog transport. games which have the same mean score but different mean square scores and hence different variances. The object of importance sampling (using non-analog probabilities) is to decrease the variance. The centra1 1 imit theorem (see eqn. (1) ) says that if $M_{2}$ can be reduced, while $M_{l}$ remains the sams, the variance will be reduced. 
IB-2 Spi"tting, Russian Roulette, and Weight Cutoff

In general, the more often a state is sampled the better the statistics associated with that state. In a given time only a certain number of particles can be followed; usually some states are undersampled while other states are oversampled. Splitting refers to replacing a particle of weight $w$ in stace $j$ by $k$ particles of weight $w / k$, thus increasing the number of times state $j$ is sampled while keeping the total weight in state $j$ constant. On the other hand, if a state is being sampled more frequently than necessary, Russian Roulette is played in which a fraction, say $1-\alpha$, of the particles are killed off and the remaining a particles are followed with their weight increased by a factor $1 / \alpha$. A Russian Roulette game cuts the number of times a state is sampled while keeping the mean weight followed in that state a constant.

A particle of very low weight is uninteresting since it cannot make much contribution to the score; yet following the particle takes time. To solve this problem a weight cutoff game is played in which a particle whose weight $w$ is less than the weight cutoff $w_{c o}$ is either killed, or followed with a new weight $w_{n}$. The particle is killed with probability $1-w / w_{n}$ and followed (with weight $w_{n}$ ) with probability $w / w_{n}$ (this is a type of Russian Roulette). 
IC Renarks on the Relationship of Chapter I to Chapter III

In the transport problems of Chapter III there is a continuum of scates a particle can occupy. The transition from Chapter I to Chapter III is largely a transition from discrete states, probabilities, and scores to continuous states, probabilities, and scores. Thus, in eqn. (33) the discrete probabilities $\tilde{p}_{i}$ and $\tilde{p}_{i j}$ will be replaced by continasus probabilities and the sum over the discrete states will be replaced by an integral over the continuum of states. The Kronecker delta function (eqn. (6) ) for discrete scores will be replaced by the Dirac delta function (eqn. (90)) for continuous scores. These delta functions serve similar purposes. Whereas the Kronecker delta function has the property (when $f$ is a function of a discrete variable)

$$
\sum_{s_{i}} f\left(s_{i}\right) \delta_{s_{i} s_{j}}=f\left(s_{j}\right),
$$

the Dirac deita function has the property (when $f$ is a function of a continuous variable)

$$
\int f(s) \delta\left(s-s^{\prime}\right) d s=f\left(s^{\prime}\right)
$$

There are five other differences between Chapter I and Chapter III. In Chapter III,

7) Particles are followed only for a finite time

8) Russian Roulette and splitting are included as events

3) A weight cutoff game is played as a particle departs an event

4) A particle may multiply (produce secondary particles as in fission)

5) The score for an event is sampled from a probability function. 
Chapter II. Definitions

This chapter contains the definitions of terms lised to derive the equations of Chapter III for the expected errors in non-analog Monte Carlo calculations of time dependent particle transport problems. It should be emphasized that these terms are for a non-analog game with particle weights, biased kernels, Russian Roulette, and splitting. It is shown later, in Chapter III, that particular choices of weights and kernels exist that make the mean score the same as in an analog game.

Definitions

$R=(\vec{r}, \vec{v})=($ particle's position, particle's velocity)

$P=(\vec{r}, \vec{v}, w)=($ particle's position, particle's velocity, particle's weight)

$P=(R, W)$

P completely describes the state of a particle in this treatment.

* is the multiplication symbol $A \star B=A B$

The probability that a particle contributes a score $s$ in ds in the next event is taken to be:

$P_{d}\left(P_{s} P^{\prime}, s\right) d s$ for a departure from $P$ followed by a collisionless

free flight to $P^{\prime}$

$P_{0}\left(P, P^{\prime}, s\right) d s$ for a departure from $P$ followed directly by an absorption at $\mathrm{P}^{\prime}$

$P_{1}\left(P, P^{\prime}, s\right) d s$ for a departure from $P$ followed directly by a scattering at $\mathrm{P}^{\prime}$ 
$P_{k}\left(P, P^{\prime}, s\right) d s$ for a departure from $P$ followed directly by a collision at $\mathrm{P}^{\prime}$ in which $k$ particles emerge from the collision

$P_{s k}\left(P_{0} P^{\prime}, s\right) d s$ for a departure from $P$ followed directly by a $k$ for one splitting event at $\mathrm{P}^{\prime}$

$P_{\rho}\left(P, P^{\prime}, S\right) d s$ for a departure from $P$ followed directly by a game of Russian Roulette at $\mathrm{P}^{\prime}$

The following weight multipliers for use in non-analog games are defined:

$w_{t}\left(R, R^{+}\right)$is the factor by which the particle's weight is multiplied if the particle makes an eventless transit from $R$ to $R^{+}$.

$W_{\sigma}\left(R^{\prime}\right)$ is the factor by which the particle's weight is multiplied if the particle has a collision at $R^{\prime}$.

$W_{\varepsilon}\left(R^{\prime}, R^{\prime \prime}\right)$ is the factor by which the particie's weight is multiplied if the particle enters a scattering event at $R^{\prime}$ and exics at $R^{\prime \prime}$.

$w_{\varepsilon_{k}}\left(R^{\prime}, R_{1}, \cdots, R_{k}\right)$ is the factor by which the $k$ secondary particles which exit a multiplying event have their weights multiplied.

$w_{c o}$ is the weight cutoff. No particle may exit a collision with weight less than $w_{\text {co }}$

$w_{e}$ is the new weight assigned to a particle if the particle survives the weight cutoff game.

The following transport kernels are defined: 


$$
T\left(P, P^{+}\right) d^{++} d w^{+}=T\left(R, R^{+}\right) \delta\left(w^{+} \cdots w, w^{+}\right) d v^{++} d^{+}
$$

$=T(\vec{r}, \vec{r}, \vec{v}) \delta\left(\vec{v}^{+}-\vec{v}\right) \delta\left(w^{+}-w_{t} w\right) d \vec{v}^{+} d w^{+}$is the probability that a particle departing $P$ will arrive at $P^{+}$, with velocity in $d^{+\vec{v}}$ and weight in $\mathrm{dw}^{+}$, without undergoing any events. Note that the velocity remains constant and the weight is multiplied by $w_{t}$ when the particle arrives at $p^{+}$.

\section{Collision Probability}

$\sigma\left(P^{+}, P^{\prime}\right) d \vec{r}^{+} d P^{\prime}=\sigma\left(R^{+}\right) \delta\left(R^{\prime}-R^{+}\right) \delta\left(W^{\prime}-W_{0} W^{+}\right) d \vec{r}^{+} d R^{\prime} d w^{\prime}$ is the probability that a particle entering $d \vec{r}$ about $\vec{r}$ will collide in $d \vec{r}$ and change state to $\mathrm{P}^{\prime}$ in $\mathrm{dP}^{\prime}$. Note that the only coordinate which changes in this process is the particle's weight, which is multiplied by $w_{\sigma^{*}} \sigma\left(R^{+}\right)$is the macroscopic total cross section at $R^{+}$.

\section{Splitting Probability}

$S_{k}\left(P^{+}\right) d \vec{r}^{+}$is the probability that a particle (at $\vec{v}^{+}$and $w^{+}$) entering $d \vec{r}$ about $\vec{r}$ will undergo a $k$ for 1 split.

\section{Russian Roulette Probahility}

$$
\begin{aligned}
& R_{0}\left(P^{+}\right) d \vec{r}^{+} \text {is the probability that a particle (at } \vec{v} \text { and } w^{+} \text {) entering } \\
& \quad \overrightarrow{d r}^{+} \text {about } \vec{r} \text { will play Russian Roulette. }
\end{aligned}
$$

\section{Collisionless Free Flight (Drift) Probability}

$$
\begin{aligned}
D\left(P, P^{+}, t\right) d P^{+}=T\left(P, P^{+}\right) \delta(|\vec{r}-\vec{r}|-v t) d P^{+} \\
=T(\vec{r}, \vec{r}, \vec{v}) \delta\left(\vec{v}^{+}-\vec{v}\right) \delta\left(w^{+}-w_{t} w\right) \delta(|\vec{r}-\vec{r}|-v t) d \vec{r}^{+} d \vec{y}^{+} d w^{+} \\
\text {is the probability that a particle departing } P \text { will have a } \\
\text { collisionless free flight (or "drift") for time } t \text { and end this }
\end{aligned}
$$


free flight in $\mathrm{dP}^{+}$about $\mathrm{P}^{+}$. Note that the velocity remains constant in free flight while the position changes by vt and the particle's weight is multiplied by $w_{t}$.

\section{Absorption Probability}

$A\left(P^{\prime}\right)$ is the probability that a collision at $P^{\prime}$ results in absorption.

\section{Scattering Probability}

$$
\begin{aligned}
& E\left(P^{\prime}, P^{\prime \prime}\right) d P^{\prime \prime} \equiv \varepsilon_{1}\left(P^{\prime}, P^{\prime \prime}\right) d P^{\prime \prime}=E\left(R^{\prime}, R^{\prime \prime}\right) \delta\left(w^{\prime \prime}-w_{\varepsilon_{1}} w^{\prime}\right) d R^{\prime \prime} d w^{\prime \prime} \\
& =E\left(\vec{v}^{\prime}, \vec{v}^{\prime \prime}\right) \delta\left(\vec{r}^{\prime \prime}-\vec{r}^{\prime}\right) \delta\left(w^{\prime \prime}-w_{\varepsilon_{1}} w^{\prime}\right) d \vec{r}^{\prime \prime} d \vec{v}^{\prime \prime} d w^{\prime \prime} \text { is the probability }
\end{aligned}
$$

that a particle colliding at $\mathrm{P}^{\prime}$ will scatter into $\mathrm{dP}$ " about P". Note that the velocity, but not the position, changes in a scattering event. The particle's weight is multiplied by $w_{\varepsilon_{1}}$ in this process.

\section{Multiplication Probability}

$$
\begin{aligned}
\varepsilon_{k}\left(P^{\prime}, P_{1}, \cdots, P_{k}\right) d P_{1} \cdots d P_{k}=\varepsilon_{k}\left(R^{\prime}, R_{1}, \cdots, R_{k}\right) \delta\left(w_{1}-w_{\varepsilon_{k}} w^{\prime}\right) \cdots \delta\left(w_{k}-w_{\varepsilon_{k}} w^{\prime}\right) \\
\quad \times d R_{1} d w_{1} \cdots d R_{k} d w_{k} \\
=\varepsilon_{k}\left(\vec{v}_{1}^{\prime}, \vec{v}_{1}, \cdots, \vec{v}_{k}\right) \delta\left(\vec{r}_{1}-\vec{r}^{\prime}\right) \cdots \delta\left(\vec{r}_{k}-\vec{r}^{\prime}\right) \delta\left(w_{1}-w_{\varepsilon_{k}} w^{\prime}\right) \cdots \delta\left(w_{k}-w_{\varepsilon_{k}} w^{\prime}\right) \\
\quad \times d \vec{r}_{1} d \vec{v}_{1} d w_{1} \cdots d \vec{r}_{k} d \vec{v}_{k} d w_{k}
\end{aligned}
$$

is the probability that a particle colliding at $\mathrm{P}^{\prime}$ results in $k$ particles, the $j^{\text {th }}$ of which $(j=1,2, \cdots, k)$ exits in $\mathrm{dP}_{j}$ about $P_{j}$. These are multiplying events such as $(n, 2 n)$ and fission. As with the scattering kernel immediately preceding, an exiting particle's weight is multiplied (here by $w_{\varepsilon_{k}}$ ' while the position is unchanged. 


\section{Weight Change Probability for Splitting}

$$
B_{s k}\left(P^{+}, P^{\prime \prime}\right) d P^{\prime \prime}=\delta(\vec{r} "-\vec{r}) \delta\left(\vec{v}^{\prime \prime}-\vec{v}\right) \delta\left(w^{\prime \prime}-w^{+} / k\right) d \vec{r}^{\prime \prime} d \vec{v}^{\prime \prime} d w^{\prime \prime}
$$

is the probability that a particle undergoing a $k$ for 1 split at $\mathrm{P}^{+}$results in $k$ particles exiting at $\mathrm{P}^{\prime \prime}$ in $d \mathrm{P}^{\prime \prime}$. Note that only the particle's weight, which is multiplien by $1 / k$, changes in a splitting event.

Weight Change Probability for Russian Roulette

$$
\begin{aligned}
& B_{0}\left(\alpha, p^{+}, P^{\prime \prime}\right) d P " d \alpha=\delta\left(\vec{r}^{\prime \prime}-\vec{r}\right) \delta\left(\vec{v}^{\prime \prime}-\vec{v}\right)\left\{\alpha \delta\left(w^{\prime \prime}-w^{+} / \alpha\right)+(1-\alpha) \delta\left(w^{\prime \prime}\right)\right\} \\
& \quad x d \vec{r}^{\prime \prime} d \vec{v}^{\prime \prime} d w^{\prime \prime} d \alpha
\end{aligned}
$$

is the probability that a particle undergoing Russian Roulette at $\mathrm{P}^{+}$(with probability $\alpha$ in da of survival) exits in dP" about P". Note that only the particle's weight changes; the particle is either killed $\left(i . e . w^{\prime \prime}=0\right.$ ) or the particle's weight is multiplied by $1 / \alpha$.

Weight Change Probability for Height Cutoff

$$
\begin{aligned}
& B\left(P^{\prime \prime}, P^{C}\right) d P^{C}=\delta\left(\vec{r}_{c}-\vec{r}^{\prime \prime}\right) \delta\left(\vec{v}_{c}-\vec{v}^{\prime \prime}\right)\left[H\left(w^{\prime \prime}-w_{c o}\right) \delta\left(w_{c}-w^{\prime \prime}\right)+\right. \\
& \left.\left(1-H\left(w^{\prime \prime}-w_{c o}\right)\right)\left\{\frac{w^{\prime \prime}}{w_{e}} \delta\left(w_{c}-w_{e}\right)+\left(1-w^{\prime \prime} / w_{e}\right) \delta\left(w_{c}\right)\right\}\right] d \vec{r}_{c} d \vec{v}_{c} d w_{c}
\end{aligned}
$$

is the probability that a particle exiting a collision at $\mathrm{P}^{\prime \prime}$ will have its coordinates changed to $\mathrm{P}^{C}$ in $\mathrm{dP}^{C}$ before the particle's next flight begins. This is the weight cutoff kernel - note that only the particle's weight changes. If the exit weight, $w^{\prime \prime}$, is greater than or equal to the cutoff weight, $w_{c o}$, the particle continues with weignt $w_{c}=w "$. If $w "<w_{c o}$ then a game of Russian 
Roulette is played in which the particle is either killed, $w_{c}=0$, or the particle is followed with weight $w_{c}=w_{e}$.

Next define the history score probability function and the $r^{\text {th }}$ moment of the history score:

$\psi(P, s, t) d s$ is the probability that a particle at $P$ will contribute

a cumulative score in ds about $s$ in the remaining time $t$.

$M_{r}(P, t)=\int \psi(P, s, t) s^{r} d s$ is the $r^{\text {th }}$ moment of the history score.

Now define

$A_{0}\left(R^{\prime}\right)=A\left(P^{\prime}\right)$ is the probability zero particles exit a collision, $A_{j}\left(R^{\prime}\right)=\int d R^{\prime \prime} E\left(R^{\prime}, R^{\prime \prime}\right)$ is the probability one particle exits a collision, and

$A_{k}\left(R^{\prime}\right)=\int d R_{1} \cdots \int d R_{k} \varepsilon_{k}\left(R^{\prime}, R_{1}, \cdots, R_{k}\right)$ is the probability $k$ particles exit a collision.

An obvious requirement is:

$$
\sum_{k=0}^{\infty} A_{k}\left(R^{\prime}\right)=1
$$

The score assigned to an event should be proportional to the particle's weight; the probability that a particle of weigh: $w^{\prime}$ contributes a score in ds about $s$ is equal to the probability that a particle of unit weight contributes a score $s / w^{\prime}$ in $d s / w^{\prime}$. Thus

$$
\begin{aligned}
& P\left(P, R^{\prime}, w^{\prime}, s\right) d s=p\left(P, R^{\prime}, l, s / w^{\prime}\right) d s / w^{\prime} \text { and } \\
& \int p\left(P, R^{\prime}, w^{\prime}, s\right) s^{r} d s=w^{\prime} \int_{p\left(P, R^{\prime}, l, s\right) s^{r} d s \text {, or }} \\
& \int p\left(P, R^{\prime}, w_{0} w, s\right) s^{r} d s=w_{0}^{r} \int p\left(P, R^{\prime}, w, s\right) s^{r} d s \text {. }
\end{aligned}
$$


This thesis also uses the notations

$H(x)=\left\{\begin{array}{ll}0 \text { if } & x<0 \\ 1 \text { if } & x \geq 0\end{array} \quad\right.$ Heaviside function

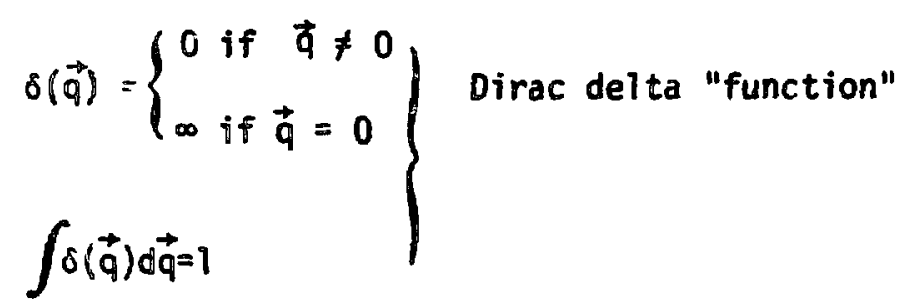

$\int_{Q} f(\vec{q}) \delta\left(\vec{q}-\vec{q}^{\prime}\right) d \vec{q}=\left\{\begin{array}{l}f\left(\vec{q}^{\prime}\right) \text { if } \vec{q}^{\prime} \text { is in the region } Q \\ 0 \text { if } \vec{q}^{\prime} \text { is not in the region } Q\end{array}\right.$

* is the multiplication operator, $A^{\star} B=A B$. 
Chapter III. Derivation of Moment Equations and Choice of Weights

In this chapter equations for the $r^{\text {th }}$ moment, $M_{r}(P, t)$, of the history score are derived. The "central linit theorem" states that the means of samples, each of $h$ histories, are, for large $h$, approximately normally distributed about $M_{1}$ with a variance

$$
\text { VAR }=\left(M_{2}-M_{1}^{2}\right) / h
$$

Thus $M_{1}$ and $M_{2}$ determine the expected error in a Monte Carlo transport calculation.

\section{A) Derivation}

The equation for $M_{r}$ is obtained by deriving a probability conservation law (eq. (91)) and multiplying this law by the $r^{\text {th }}$ power of the history score, $\mathrm{s}^{r}$, and integrating over all possible scores. Let $t^{+}=t^{\prime}$ be the time required for a free flight from $P$ to $P^{+}$. (Since a collision process requires no time, $t^{\prime}$ may be interpreted as the time between departing $P$ and a collision at $\left.P^{\prime}.\right)$ Note that $\psi(P, s, t) d s$ is the probability of obtaining a score in ds about $s$ in the time $t$ remaining; a particle departing $P$ is limited in its possible next event points to those states $P^{+}$that can be reached from $P$ in time $t^{+}<t$. This is the reason for the $t^{+}<t$ limitation on the integrations over $\mathrm{p}^{+}$in eq. (91). The first term in eq. (91) (see page 36) has a $t^{+}=t$ restriction on the integral over $p^{+}$since this term is due to a free flight for the entire time $t$; thus the states $\mathrm{P}^{+}$are limited to 
those states that can be reached in exactly time $t$.

In order to obtain a history score $s$ in ds a particle that obtains score $s^{\prime}$ in the next event must obtain a cumulative score in ds about $s-s^{\prime}$ in ail subsequent events. If there are no subsequent events, as in TERMI and TERM2 of eq. (91), then the entire history score must be contributed at the next event, i.e. $s^{\prime}=s$ in ds.

There are six possible ways a particle departing $P$ can end its free fiught:

1) run the clock out (free flight for the entire time $t$ )

2) absorption at $P^{\prime}$

3) play Russian Roulette at $\mathrm{P}^{+}$

4) split at $p^{+}$

5) scatter at $P^{\prime}$

6) multiply at $p^{\prime}$

Each of the six terms in eq. (91) is a product of probabilities of the form

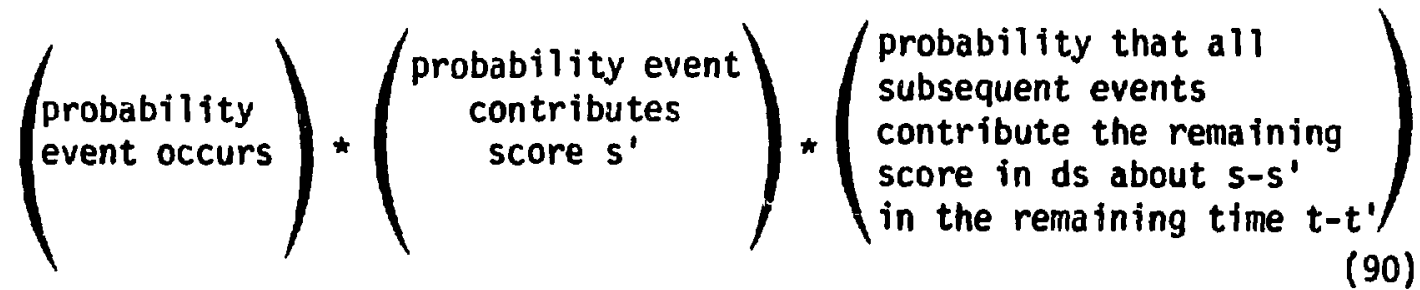

Each of these three factors will be listed for each of the six terms of eq. (91). After these factors have been listed a detailed explanation of eq. (91) will be given. 
1) Free flight for remaining time $t$

$$
\left(D\left(P, P^{+}, t\right) d P^{+}\right) x\left(p_{d}\left(P, P^{+}, s^{\prime}\right) d s^{\prime}\right) x\left(\delta\left(s-s^{\prime}\right) d s\right)
$$

2) Absorption (free flight to $\mathrm{P}^{+}$, collision in $\mathrm{dP}^{+}$, absorption at $\mathrm{P}^{\prime}$ ) $\left(T\left(P, P^{+}\right) \sigma\left(P^{+}, P^{\prime}\right) d P^{+} d P^{\prime} A\left(P^{\prime}\right)\right) \times\left(P_{0}\left(P, P^{\prime} s^{\prime}\right) d s^{\prime}\right) \times\left(\delta\left(s-s^{\prime}\right) d s\right)$

3) Russian Rouiette (free $f 1$ ight to $\mathrm{P}^{+}$, Russian Roulette game at $\mathrm{P}^{+}$, exit Russian Roulette game at P") $\left(T\left(P, P^{+}\right) R_{0}\left(P^{+}\right) d P^{+} B_{0}\left(\alpha, P^{+}, P^{\prime \prime}\right) d \alpha d P^{\prime \prime}\right) \times\left(P_{\rho}\left(P, P^{+}, s^{\prime}\right) d s^{*}\right)$ $x\left(\psi\left(P^{\prime \prime}, s-s^{\prime}, t-t^{\prime}\right) d s\right)$

4) Splitting (free flight to $\mathrm{P}^{+}$, split $k$ for 1 at $\mathrm{P}^{+}$, exit splitting at P")

$$
\begin{aligned}
& \left(T\left(P, P^{+}\right) s_{k}\left(P^{+}\right) d P^{+} B_{s k}\left(P^{+}, P^{\prime \prime}\right) d P^{\prime \prime}\right) \times\left(P_{s k}\left(P, P^{+}, s^{\prime}\right) d s^{\prime}\right) \\
& x\left(\psi\left(P^{\prime \prime}, s_{1}, t-t^{+}\right) d s_{1} \cdots \psi\left(P^{\prime \prime}, s_{k-1}, t-t^{+}\right) d s_{k-1}\right. \\
& \left.x \psi\left(P^{\prime \prime}, s-\left(s^{\prime}+s_{1}+\cdots+s_{k-1}\right), t-t^{+}\right) d s\right)
\end{aligned}
$$

5) Scattering (free flight to $\mathrm{P}^{+}$, collision in $\mathrm{dP}^{+}$, exit collision in dP", Russian Roulette game at P", exit Russian Roulette game in $\left.d p^{c}\right)$

$$
\begin{aligned}
& \left(T\left(P, P^{+}\right) \sigma\left(P^{+}, P^{\prime}\right) d P^{+} d P^{\prime} E\left(P^{\prime}, P^{\prime \prime}\right) d P^{\prime \prime} B\left(P^{\prime \prime}, P^{C}\right) \times\left(P_{1}\left(P, P^{\prime}, s^{\prime}\right) d s^{\prime}\right)\right. \\
& x\left(\psi\left(P^{C}, s-s^{\prime}, t-t^{\prime}\right) d s\right)
\end{aligned}
$$

6) Muitiplying (free flight to $\mathrm{P}^{+}$, collision in $\mathrm{dP}^{+}, k$ particles exit collision in $\mathrm{dP}_{1}, \cdots, \mathrm{dP} \mathrm{P}_{k}$, Russian Roulette games at $\mathrm{P}_{1}, \cdots, \mathrm{P}_{k}$, exit Russian Roulette games in $\mathrm{dP}_{1}^{c}, \cdots, \mathrm{dP} \mathrm{C}_{k}$ )

$$
\begin{aligned}
& \left(T\left(P, P^{+}\right) \sigma\left(P^{+}, P^{\prime}\right) d P^{+} d P^{\prime} \varepsilon_{k}\left(P^{\prime}, P_{1}, \cdots, P_{k}\right) d P_{1}, \ldots d P_{k} B\left(P_{1}, P_{1}^{C}\right) d P_{1}^{C} \ldots\right. \\
& \left.x B\left(P_{k}, P_{k}^{C}\right) d P_{k}^{c}\right) x\left(P_{k}\left(P^{\prime}, P^{\prime}, s^{\prime}\right) d s^{\prime}\right) \times\left(\psi\left(P_{1}^{C}, s_{1}, t-t^{\prime}\right) d s_{1} \ldots\right. \\
& \left.x \psi\left(P_{k-1}^{C}, s_{k-1}, t-t^{\prime}\right) d s_{k-1} \psi\left(P_{k}^{C}, s-\left(s^{\prime}+s_{1}+\cdots+s_{k-1}\right), t-t^{\prime}\right) d s\right)
\end{aligned}
$$


The score accumulation probability $\psi(P, s, t) d s$ is separated into the six terms given on the right hand side of eq. (91). These six terms are score accumulation probabilities for particles that end their first flights from $P$ by:

1) a collisionless free flight for the remaining time $t$

2) being absorbed at $P^{\prime}$

3) playing Russian Roulette at $\mathrm{p}^{+}$

4) splitting at $\mathrm{P}^{+}$

5) scattering at $P^{\prime}$

6) multiplying at $P^{\prime}$

In TERMI $D\left(P, P^{+}, t\right) d^{+}$of the particles departing $P$ arrive in $d P^{+}$about $P^{+}$in exactly time $t$ without undergoing any events and $P_{d}\left(P, P^{+}, s^{\prime}\right) d s^{\prime}$ of the $D\left(P, P^{+}, t\right) d P^{+}$particles contribute a score $s^{\prime}$ in $d s^{\prime}$; and $\delta\left(s^{\prime}-s\right) d s$ of the $D\left(P, P^{+}, t\right) d P^{+} P_{d}\left(P, P^{\prime}, s^{\prime}\right) d s^{\prime}$ particles contribute a score in ds about $s$. The history score, s, for a particle departing $P$ which undergoes no events in the entire time $t$ consists only of the single score $s$ ' due to the free flight of the particle; thus the history score will be in ds about $s$ if and only if $s^{\prime}$ is in ds about $s$, hence the delta function.

In TERM2 $T\left(P, P^{+}\right)$of the particles departing $P$ make a free flight to $P^{+}$. $\sigma\left(P^{+}, P^{\prime}\right) d P^{+} d P^{\prime}$ of the $T\left(P, P^{+}\right.$) particles collide in $\mathrm{dP}^{+}$and have their state changed (only the weight coordinate changes) to $\mathrm{P}^{\prime}$ in $\mathrm{dP}^{\prime}$. $A\left(\mathrm{P}^{\prime}\right)$ of the $T\left(P_{,}, \mathrm{P}^{+} \partial \sigma\left(\mathrm{P}^{+}, \mathrm{P}^{\prime}\right) \mathrm{dP}^{+} \mathrm{dP}^{\prime}\right.$ particles are absorbed at $P^{\prime}$. $P_{0}\left(P, P^{\prime}, s^{\prime}\right) d s^{\prime}$ of the $T\left(P, P^{+}\right) \sigma\left(P^{+}, P^{\prime}\right) d P^{+} d P^{\prime} A\left(P^{\prime}\right)$ particles contribute a score $s^{\prime}$ in $d s^{\prime}$. The history score, $s$, for a particle that is absorbed in its first event consists only of the single score s' 
due to the absorption; thus the history score will be in ds about s if and only if $s^{\prime}$ is in ds about $s$. Hence $T\left(P, P^{+}\right) \sigma\left(P^{+}, P^{\prime}\right) d P^{+} d P^{\prime} A\left(P^{\prime}\right)$ $x \delta\left(s-s^{\prime}\right) d s$ of the particies departing $P$ contribute a score in ds about $s$ by being absorbed in the next event.

In TERM3 $T\left(P, P^{+}\right)$of the particles departing $P$ free flight to $P^{+}$. $R_{0}\left(P^{+}\right) d^{+}$of the $T\left(P, P^{+}\right)$particles play Russian Roulette in $\mathrm{dP}^{+}$. $B_{0}\left(\alpha, P^{+}, P^{\prime \prime}\right) d \alpha d P^{\prime \prime}$ of the $T\left(P, P^{+}\right) R_{0}\left(P^{+}\right) d P^{+}$particles exit the Russian Roulette game in $d P^{\prime \prime}$. $P_{\rho}\left(P, P^{+}, s^{\prime}\right) d s^{\prime}$ of the $T\left(P, P^{+}\right) R_{0}\left(P^{+}\right) d P^{+} B_{0}\left(\alpha, P^{+}, P^{\prime \prime}\right)$ $x$ dadp" particles contribute a score $s^{\prime}$ in $\mathrm{ds}^{\prime}$ at this event. $\psi\left(P^{\prime \prime}, s-s^{\prime}, t-t^{+}\right) d s$ of the $T\left(P, P^{+}\right) R_{0}\left(P^{+}\right) d P^{+} B_{0}\left(\alpha, P^{+}, P^{\prime \prime}\right) d \alpha d P^{\prime \prime} P_{p}\left(P, P^{+}, s^{\prime}\right) d s^{\prime}$ particles subsequently contribute cumulative scores in ds about s-s' to make the history score in ds about $s^{1}+\left(s-s^{0}\right) r s$. Thus $T\left(P_{s} P^{+}\right) R_{0}\left(P^{+}\right)$ $x d P^{+} B_{0}\left(a, P^{+}, P^{\prime \prime}\right) d a d P^{\prime \prime} p_{\rho}\left(P, P^{+}, s^{\prime}\right) d s^{\prime} \psi\left(p^{\prime \prime}, s-s^{\prime}, t-t^{+}\right) d s$ of the particles departing $P$ contribute a score in ds about $s$ by having the next event be a Russian Rouletre game.

TERMA $T\left(P, P^{+}\right)$of the particles departing $P$ free flight to $P^{+}$. $S_{k}\left(P^{+}\right) d P^{+}$of the $T\left(P, P^{+}\right)$particles undergo a $k$ for 1 split in $\mathrm{dP}^{+}$. $B_{s k}\left(P^{+}, P^{\prime \prime}\right) d P^{\prime \prime}$ collections of $k$ particles exit the $T\left(P, P^{+}\right) S_{k}\left(P^{+}\right) d P^{+}$ splitting events (into $\left.d P^{\prime \prime}\right) . p_{s k}\left(P, P^{+}, s^{\prime}\right) d s^{\prime}$ of the $T\left(P, P^{+}\right) s_{k}\left(P^{+}\right) d P^{+}$ $\mathrm{XB}_{s k}\left(P^{+}, P^{\prime \prime}\right) d P^{\prime \prime}$ splitting events contribute a score $s^{\prime}$ in $d s^{\prime}$.

$$
\begin{aligned}
& \int \psi\left(P^{\prime \prime}, s_{1}, t-t^{+}\right) d s_{1} \cdots \int \psi\left(P^{\prime \prime}, s_{k-1}, t-t^{+}\right) d s_{k-1} \\
& x \psi\left\langle P^{\prime \prime}, s_{-}\left(s^{\prime}+s_{1}+\cdots+s_{k-1}\right), t-t^{+}\right) d s
\end{aligned}
$$

of the collections of $k$ particles subsequentiy contribute the remaining 
score, $s_{j}+s_{2}+\cdots+s_{k-1}+\left[s-\left(s^{\prime}+s_{j}+\cdots+s_{k-1}\right)\right]=s-s^{\prime}$ 。 to make the history score in ds about $s^{0}+s_{1}+\cdots+s_{k-1}+\left[s-\left(s^{0}+s_{j}+\cdots+s_{k-1}\right)\right]=s$. Restating this last idea, each of the $T\left(P, P^{+}\right) S_{k}\left(P^{+}\right) d P^{+} B_{s k}\left(P^{+}, P^{\prime \prime}\right) d P^{\prime \prime} P_{s k}\left(P, P^{+}, s^{\prime}\right) d s^{\prime}$ collections of $k$ particles exiting $P$ " has already contributed a score $s$ ' of the desired history score $s ;$ thus the $k$ particles exiting p" must collectively contribute a cumulative score in ds about s-s' in the remaining time $t-t^{+}$. The probability that $k$ particles departing $P^{\prime \prime}$ collectively contribute a cumulative score in ds about s-s in time $t-t^{+}$ is

$$
\begin{aligned}
& \int d s_{1} \cdots \int d s_{k-1} \psi\left(P ", s_{1}, t-t^{+}\right) \cdots \psi\left(P^{\prime \prime}, s_{k-1}, t-t^{+}\right) \\
& x \psi\left(P^{\prime \prime}, 5-\left(s^{+}+s_{1}+\cdots+s_{k-1}\right), t-t^{+}\right) d s
\end{aligned}
$$

That is, if particles 1 through $k-i$ contribute scores $s_{j}$ through $s_{k-1}$, then the $k^{\text {th }}$ particle must contribute a score in $d s$ about $s-\left(s^{0}+s_{1}+00+s_{k-1}\right)$ so that the score $s^{\prime}$, due to splitting, plus the scores due to the $k$ particies departing dp" results in a total score

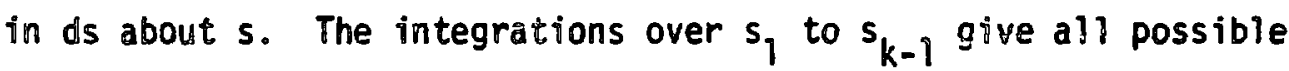
ways that $s_{j}$ through $s_{k}$ can sum to $s-s^{\prime}$.

In TERM5 $T\left(P, P^{+}\right)$of the particles departing $P$ free flight to $P^{+}$. $\sigma\left(P^{+}{ }_{0} P^{\prime}\right) d P^{+} d P^{\prime}$ of the $T\left(P_{0} P^{+}\right)$particles collide in $d P^{+}$and change state (only the weight coordinate changes) to $\mathrm{P}^{\prime}$ in $\mathrm{dP}$ '. $E\left(P^{\prime}, P^{\prime \prime}\right) d P^{\prime \prime}$ of the $T\left(P, P^{+}\right) \sigma\left(P^{+}, P^{\prime}\right) d P^{+} d P^{\prime}$ particles exit the collision in $d P^{\prime \prime} . B\left(P^{\prime \prime}{ } P^{C}\right) d P^{C}$ of the $T\left(P, P^{+}\right) \sigma\left(P^{+}, P^{\prime}\right) d P^{+} d P^{\prime} E\left(P^{\prime}{ }^{\prime} P^{\prime \prime}\right) d P^{\prime \prime}$ particles playing the weight cutoff game at $P$ " exit the game in $d P^{\mathbb{C}}$. 
$P_{1}\left(P, P^{\prime}, s^{\prime}\right) d s^{\prime}$ of the $T\left(P, P^{+}\right) \sigma\left(P^{+}, P^{\prime}\right) d P^{+} d P^{\prime} E\left(P^{\prime}, P^{\prime \prime}\right) d P^{\prime \prime} B\left(P^{\prime \prime}, P^{C}\right) d P^{C}$ particles contribute a score $s^{\prime}$ (in ds') in the scattering event. $\psi\left(P^{c}, s-s^{\prime}, t-t^{\prime}\right) d s$ of the $T\left(P, P^{+}\right) \sigma^{\prime}\left(P^{+}, P^{\prime}\right) d P^{+} d P^{\prime} E\left(P^{\prime}, P^{\prime \prime}\right) d P^{\prime \prime} B\left(P^{\prime \prime}, P^{c}\right) d P^{C}$ $\times P_{1}\left(P, P^{\prime}, s^{\prime}\right) d s^{\prime}$ particles departing $P^{C}$ subsequently contribute the remaining score in ds about $s-s^{\prime}$ in the remaining time $t-t$ ' so that the total history score is $s^{\prime}+\left(s-s^{\prime}\right)=s$ in $d s$.

In TERM6 $T\left(P, P^{+}\right)$of the particles departing $P^{\prime}$ free flight to $P^{+}$. $\sigma\left(P^{+}, P^{\prime}\right) d P^{+} d P^{\prime}$ of the $T\left(P, P^{+}\right)$particles collide in $d P^{+}$and change state (only the weight coordinate changes) to $\mathrm{P}^{\prime}$ in $\mathrm{dP}$ '. $\varepsilon_{k}\left(P^{\prime}, P_{l}, \cdots, P_{k}\right) d P_{l} \cdots d P_{k}$ collections of $k$ particles exit (the $T\left(P, P^{+}\right) \sigma\left(P^{+}, P^{\prime}\right) d P^{+} d P^{\prime}$ collisions in $\left.d P^{+}\right)$in $d P_{1}, \cdots, d P_{k^{\circ}}$ $B\left(P_{1}, P_{1}^{C}\right) d P_{1}^{C} \cdots B\left(P_{k}, P_{k}^{C}\right) d P_{k}^{C}$ of the $T\left(P, P^{+}\right) \sigma\left(P^{+}, P^{\prime}\right) d P^{+} d P^{\prime} \varepsilon_{k}\left(P^{\prime}, P_{1}, \cdots, P_{k}\right)$ $x \mathrm{dP}_{f} \cdots \mathrm{dP}_{k}$ collections of $k$ particles playing the weight cutoff game at $P_{j}, \cdots{ }_{2} P_{k}$ exit the game in $d P_{j}^{c}, \cdots, d P_{k}^{c}, p_{k}\left(P, P^{\prime}, s^{\prime}\right) d s^{\prime}$ of the $T\left(P, P^{+}\right) \sigma\left(P^{+}, P^{\prime}\right) d P^{+} d P^{\prime} \varepsilon_{k}\left(P^{\prime}, P_{j}, \cdots, P_{k}\right) d P_{j} \cdots d P_{k}$ $\times B\left(P_{j}, P_{j}^{C}\right) d P_{j}^{C} \cdots B\left(P_{k}, P_{k}^{C}\right) d P_{k}^{C}$ collections of $k$ particles departing $p_{j}^{c}, \ldots, p_{k}^{c}$ contribute a score $s^{\prime}$ (in ds') in the multipiying event. $\int \psi\left(P_{1}^{c}, s_{j}, t-t^{\prime}\right) d s_{1} \cdots \int \psi\left(P_{k-1}^{c}, s_{k-1}, t-t^{\prime}\right) d s_{k-1}$ $x \psi\left(p_{k}^{C}, s-\left(s_{1}+s^{1}+\cdots+s_{k-1}\right), t-t_{1}\right) d s$

of the collections of $k$ particles contribute the remaining score $s_{7}+s_{2}+\cdots+s_{k-1}+\left[s-\left(s^{0}+s_{1}+\cdots+s_{k-j}\right)\right]=s-s^{\prime}$ in the remaining time $t-t^{\prime}$, to make the history score in ds about $s^{\prime}+s_{1}+\cdots+s_{k-1}+\left[\left(s-\left(s^{0}+s_{j}+\cdots+s_{k-1}\right)\right]=s\right.$. Restating this last idea, each of the $T\left(P, P^{+}\right) \sigma\left(P^{+}, P^{\prime}\right) d P^{+} d P^{\prime} P_{k}\left(P_{,} P^{\prime}, s^{1}\right) d^{\prime} s^{\prime}$ 
$\times \varepsilon_{k}\left(P^{\prime}, P_{1}, \cdots, P_{k}\right) d P_{1} \cdots d P_{k} B\left(P_{1}, P_{1}^{C}\right) d P_{1}^{C} \cdots B\left(P_{k}, P_{k}^{C}\right) d P_{k}^{C}$ collections of $k$ particles exiting $P_{j}^{c}, \cdots, P_{k}^{c}$ has already contributed a score $s '$ of the desired history score $s$; thus the $k$ particles exiting $p_{j}^{c}, \cdots, p_{k}^{c}$ must collectively contribute a cumulative score in as about sss' in the remaining time $t-t^{\prime}$. The probability that $k$ particles departing $P_{1}^{c}, \cdots, p_{k}^{c}$ collectively contribute a cumulative score in as about sss' in time $t-t^{\prime}$ is

$$
\begin{aligned}
& \int \psi\left(P_{1}^{c}, s_{1}, t-t^{\prime}\right) d s_{1} \cdots \int \psi\left(P_{k-1}^{c}, s_{k-1}, t-t^{\prime}\right) d s_{k-1} \\
& \times \psi\left(P_{k}^{c}, s-\left(s^{\prime}+s_{1}+\cdots+s_{k-1}\right), t-t^{\prime}\right) d s
\end{aligned}
$$

That is, if particles 1 through $k-1$ contribute scores $s_{1}$ through $s_{k-1}$, then the $k^{\text {th }}$ particle must contribute a score in os about $s-\left(s^{\prime}+s_{1}+\cdots+s_{k-1}\right)$ so that the score $s^{\prime}$, due to multiplication, plus the scores due to the $k$ particles exiting $P_{1}^{c}, \cdots, p_{k}^{c}$ results in a total score in as about $s$. The integrations over $s_{1}$ to $s_{k-1}$ give all possible ways that $s_{j}$ through $s_{k}$ can sum to sis'.

Adding TERMI through TERM 6 results in the final equation for the probability of obtaining score $s$ in the time $t$ remaining

$$
\begin{aligned}
& \psi(P, s, t) d s=\int_{t^{+}=t} d P^{+} D\left(P, P^{+}, t\right) \int d s^{\prime} P_{d}\left(P, P^{+}, s^{\prime}\right) \delta\left(s^{\prime} s^{\prime}\right) d s \\
& +\int_{t^{+}<t} d P^{+} T\left(P, P^{+}\right) \int d P^{\prime} \sigma\left(P^{+}, P^{\prime}\right) \int d s^{\prime} P_{0}\left(P, P^{\prime}, s^{\prime}\right) A\left(P^{\prime}\right) \delta\left(s-s^{\prime}\right) d s \\
& +\int_{t^{+}<t} d P^{+} T\left(P, P^{+}\right) R_{0}\left(P^{+}\right) \int d s^{\prime} P_{p}\left(P, P^{+}, s^{\prime}\right) \int d P^{\prime \prime} \int_{0}^{1} d \alpha B_{0}\left(\alpha, P^{+}, P^{\prime \prime}\right) \\
& \times \psi\left(P^{\prime \prime}, s-s^{\prime}, t-t^{+}\right) d s+\sum_{k=2}^{\infty} t^{+}<t d P^{+} T\left(P, P^{+}\right) s_{k}\left(P^{+}\right) \int d s^{\prime} P_{s k}\left(P, P^{+}, s^{\prime}\right) \\
& \times \int d P^{\prime \prime} B_{s k}\left(P^{+}, P^{\prime \prime}\right) \int d s_{1} \ldots \int d s_{k-1} \psi\left(P^{\prime \prime}, s_{1}, t-t^{+}\right) \ldots \psi\left(P^{\prime \prime}, s_{k-1}, t-t^{+}\right)
\end{aligned}
$$

35 


$$
\begin{aligned}
& x \psi\left(P^{\prime \prime}, s^{-}\left(s^{1}+s_{1}+\cdots+s_{k-1}\right), t-t^{+}\right) d s+\int_{t}^{+}<t \\
& x \int d s^{\prime} P_{1}\left(P_{2}, P^{\prime}, s^{\prime}\right) \int d P^{\prime \prime} E\left(P^{\prime}, P^{n}\right) \int d P^{C} B\left(P^{\prime \prime}, P^{c}\right) \psi\left(P^{c}, s-s^{\prime}, t-t^{\prime}\right) d s \\
& +\sum_{k=2}^{\infty} t_{t}^{+} \int_{<t} d P^{+} T\left(P_{,}, P^{+}\right) \int d P^{\prime} \sigma\left(P^{+}, P^{\prime}\right) \int d s^{\prime} P_{k}\left(P, P^{\prime}, s^{\prime}\right) \\
& \times \int d P_{1} \cdots \int d P_{k} \varepsilon_{k}\left(P^{\prime}, P_{1}, \cdots, P_{k}\right) \int d P_{1}^{C} B\left(P_{1}, P_{1}^{c}\right) \ldots \int d P_{k}^{c_{B}}\left(P_{k}, P_{k}^{c}\right) \int d s_{1} \ldots \int d s_{k-1} \\
& x \psi\left(P_{1}^{c}, s_{1}, t-t^{\prime}\right) \cdots \psi\left(P_{k-1}^{c}, s_{k-1}, t-t^{\prime}\right) \psi\left(P_{k}^{c}, s^{-}\left(s^{\prime}+s_{1}+\cdots+s_{k-1}\right), t-t^{\prime}\right) d s
\end{aligned}
$$

This equation is of the form

$$
\text { TERM }=\sum_{I=1}^{6} \text { TERM }
$$

Multiplying eq. (91) by $s^{r}$ and integrating over $s$ results in the desired equation for $M_{r}$. The integrations are done term by term below.

$$
\begin{aligned}
\text { TERM } & =\int d s \psi(P, s, t) s^{r}=M_{r}(P, t) \\
\text { TERM 1 } & =\int_{t}^{+} \int_{=t} d P^{+} D\left(P, P^{+}, t\right) \int d s p_{d}\left(P, P^{+}, s\right) s^{r} \\
\text { TERM } & =\int_{t}^{+} d P^{+} T\left(P, P^{+}\right) \int d P^{\prime} \sigma\left(P^{+}, P^{\prime}\right) \int d s p_{0}\left(P, P^{\prime}, s\right) A\left(P^{\prime}\right) s^{r} \\
\text { TERM } & =\int_{t}^{+} \int d P^{+} T\left(P, P^{+}\right) R_{0}\left(P^{+}\right) \int d s^{\prime} P_{\rho}\left(P, P^{+}, s^{\prime}\right) \int d P^{\prime \prime} \int_{0}^{T} d \alpha B_{0}\left(\alpha, P^{+}, P^{\prime \prime}\right) \\
& x \int d s \psi\left(P^{\prime \prime}, s-s^{\prime}, t-t^{+}\right) s^{r}
\end{aligned}
$$

Using eq. (A5)

$$
\begin{aligned}
\text { TERM 3 } & =\int_{t}^{+}<t \\
& \times \sum_{n=0}^{r}\left(P^{+} T\left(P, P^{+}\right) R_{0}\left(P^{+}\right) \int d s^{\prime} P_{\rho}\left(P, P^{+} P_{n}, s^{\prime}\right) \int d P^{\prime \prime} \int_{0}^{1} d \alpha B_{0}\left(\alpha, t^{+}\right)\right.
\end{aligned}
$$

36 


$$
\begin{aligned}
& \text { TERM4 }=\sum_{k=2}^{\infty} \int_{t} \int_{<t} d P^{+} T\left(P, P^{+}\right) S_{k}\left(P^{+}\right) \int d s^{\prime} P_{s k}\left(P_{2} P^{+}, s^{\prime}\right) \int d P^{\prime \prime} B_{s k}\left(P^{+}, P^{\prime \prime}\right) \\
& x \int d s_{1} \cdots \int d s_{k-1} \psi\left(P^{\prime \prime}, s_{1}, t-t^{+}\right) \ldots \psi\left(P^{\prime \prime}, s_{k-1}, t-t^{+}\right) \int d s^{r} \\
& x \psi\left(P^{\prime \prime}, s-\left(s^{\prime}+s_{1}+\cdots+s_{k-1}\right), t-t^{+}\right)
\end{aligned}
$$

Using eq. (A5) on $\int d s$

$$
\begin{aligned}
& \text { TERMQ }=\sum_{k=2}^{\infty} t_{t}^{+} \int_{<t} d P^{+} T\left(P, P^{+}\right) s_{k}\left(P^{+}\right) \int d s^{\prime} P_{s k}\left(P, P^{+}, s^{0}\right) \int d P^{\prime \prime} B_{s k}\left(P^{+}, P^{\prime \prime}\right) \\
& \times \int d s_{1} \cdots \int d s_{k-1} \psi\left(P^{\prime \prime}, s_{1}, t-t^{+}\right) \cdots \psi\left(P^{\prime \prime}, s_{k-1}, t-t^{+}\right) \\
& \times \sum_{n_{1}=0}^{r}\left(\begin{array}{l}
r \\
n_{1}
\end{array}\right)\left(s^{+}+s_{1}+\cdots+s_{k-1}\right)^{r-n_{1}} M_{n_{1}}\left(P^{\prime \prime}, t-t^{+}\right)
\end{aligned}
$$

Using eq. (A5) on $\int d s_{k-1}$

$$
\begin{aligned}
& \text { TERM4 }=\sum_{k=2}^{\infty} t_{t^{+}}^{\infty} d P^{+} T\left(P, P^{+}\right) s_{k}\left(P^{+}\right) \int d s^{\prime} P_{s k}\left(P, P^{+}, s^{\prime}\right) \int d P^{\prime \prime} B_{s k}\left(P^{+}, P^{\prime \prime}\right) \\
& x \int d s_{1} \cdots \int d s_{k-2} \psi\left(P^{\prime \prime}, s_{1}, t-t^{+}\right) \ldots \psi\left(P^{\prime \prime}, s_{k-2}, t-t^{+}\right) \sum_{n_{1}=0}^{r}\left(\begin{array}{l}
r \\
n_{1}
\end{array}\right) M_{n_{1}}\left(P^{\prime \prime}, t-t^{+}\right) \\
& x \sum_{n_{2}=0}^{r-n_{1}}\left(\begin{array}{c}
r-n_{1} \\
n_{L}
\end{array}\right) M_{n_{2}}\left(P^{\prime \prime}, t-t^{+}\right)\left(s^{\prime}+s_{1}+\ldots+s_{k-2}\right)^{r-n_{1}-n_{2}}
\end{aligned}
$$

Using eq. (A5) repeatedly on $\int d s_{k-2} \cdots \int d s_{1}$

$$
\begin{aligned}
& \text { TERM4 }=\sum_{k=2}^{\infty} t_{t}^{+}<t d P^{+} T\left(P, P^{+}\right) S_{k}\left(P^{+}\right) \int d s^{\prime} P_{s k}\left(P, P^{+}, s^{\prime}\right) \int d P^{\prime \prime} B_{s k}\left(P^{+}, P^{\prime \prime}\right) \\
& x \sum_{n_{1}=0}^{r}\left(\begin{array}{l}
r \\
n_{1}
\end{array}\right) M_{n_{1}}\left(P^{\prime \prime}, t-t^{+}\right) \cdots \sum_{n_{k}=0}^{r-n_{1}-\cdots-n_{k-1}}\left(\begin{array}{c}
r-n_{1} \cdots-n_{k-1} \\
n_{k}
\end{array}\right) M_{n_{k}}\left(P^{\prime \prime}, t-t^{+}\right) \\
& \times s^{r-n_{1} \cdots \cdots-n_{k}}
\end{aligned}
$$

(100)

37 


$$
\begin{aligned}
& \text { TERM 5 }=t_{t}^{+} \int_{<t} d P^{+} T\left(P_{2}, P^{+}\right) \int d P^{\prime} \sigma\left(P^{+}, P^{\prime}\right) \int d s^{\prime} P_{1}\left(P, P^{\prime}, s^{\prime}\right) \int d P^{\prime \prime} E\left(P^{\prime}, P^{\prime \prime}\right) \\
& x \int d P^{c} B\left(P^{\prime \prime}, P^{c}\right) \int d s \psi\left(P^{c}, s-s^{\prime}, t-t^{\prime}\right) s^{r}
\end{aligned}
$$

Using eq. (A5)

$$
\begin{aligned}
& \text { TERMs }={ }_{t}^{+}<t d P^{+} T\left(P, P^{+}\right) \int d P^{\prime} \sigma\left(P^{+}, P^{\prime}\right) \int d s^{\prime} P_{1}\left(P^{\prime}, P^{\prime}, s^{\prime}\right) \int d P^{\prime \prime} E\left(P^{\prime}, P^{\prime \prime}\right) \\
& x \int d P^{c} B\left(P^{* i}, P^{c}\right) \sum_{n=0}^{r}\left(\begin{array}{l}
r \\
n
\end{array}\right) M_{n}\left(P^{c}, t-t^{\prime}\right) s^{\prime}, r-n \\
& \text { TERMs }=\sum_{k=2}^{\infty} t_{t}^{+} \int_{<t} d P^{+} T\left(P, P^{+}\right) \int d P^{\prime} \sigma\left(P^{+}, P^{\prime}\right) \int d s^{\prime} P_{k}\left(P, P^{\prime}, s^{\prime}\right) \\
& \times \int d P_{1} \cdots \int d P_{k} \varepsilon_{k}\left(P^{\prime}, P_{1}, \cdots, P_{k}\right) \int d P_{1}^{C} B\left(P_{1}, P_{1}^{C}\right) \cdots \int d P_{k}^{C} C_{k}\left(P_{k}, P_{k}^{C}\right) \\
& x \int d s_{1} \cdots \int d s_{k-1} \psi\left(P_{1}^{c}, s_{1}, t-t^{\prime}\right) \cdots \psi\left(P_{k-1}^{c}, s_{k-1}, t-t^{\prime}\right) \int d s \\
& x \psi\left(P_{k}^{c}, s-\left(s^{\prime}+s_{1}+\cdots+s_{k-1}\right), t-t^{\prime}\right) s^{r}
\end{aligned}
$$

Using eq. (A5) repeatedly as in TERM4

$$
\begin{aligned}
& \text { TERMs }=\sum_{k=2}^{\infty} t_{t}^{+} \int_{t} d P^{+} T\left(P, P^{+}\right) \int d P^{\prime} \sigma\left(P^{+}, P^{\prime}\right) \int d s^{\prime} P_{k}\left(P, P^{\prime}, s^{\prime}\right) \int d P_{1} \cdots \int d P_{k} \\
& x \varepsilon_{k}\left(P^{\prime}, P_{1}, \ldots, P_{k}\right) \int d P_{1}^{C} B\left(P_{\gamma}, P_{1}^{C}\right) \ldots \int d P_{k}^{C_{B}}\left(P_{k}, P_{k}^{C}\right) \\
& \times \sum_{n_{1}=0}^{r}\left(\begin{array}{l}
r \\
n_{1}
\end{array}\right) M_{n_{1}}\left(P_{k}^{c}, t-t^{\prime}\right) \sum_{n_{2}=0}^{r-n_{1}}\left(\begin{array}{c}
r-n_{1} \\
n_{2}
\end{array}\right) M_{n_{2}}\left(P_{k-1}^{c}, t-t^{\prime}\right) \ldots \\
& \times \sum_{n_{k}=0}^{r-n_{1} \cdots-n_{k-1}}\left(\begin{array}{c}
r-n_{1} \cdots \cdots-n_{k-1} \\
n_{k}
\end{array}\right) M_{n_{k}}\left(p_{1}^{c}, t-t^{\prime}\right) s^{\prime}{ }^{r-n_{1} \cdots \cdots-n_{k}}
\end{aligned}
$$

Changing the dummy variable $s^{\prime}$ to the dummy variable $s$ and adding TERM $=\sum_{I=1}^{6}$ TERM

gives the equation for the $r^{\text {th }}$ moment

38 


$$
\begin{aligned}
& M_{r}(P, t)=\int_{t^{+}=t} d P^{+} D\left(P, P^{+}, t\right) \int d s p_{d}\left(P, P^{+}, s\right) s^{r} \\
& +\int_{t}^{+} \int_{\varepsilon_{\imath}} d P^{+} T\left(P, P^{+}\right) \int d P^{\prime} \sigma\left(P^{+}, P^{\prime}\right) \int d s P_{0}\left(P_{,} P^{\prime}, s\right) A\left(P^{\prime}\right) s^{r} \\
& +\int_{t}^{+}<t d P^{+} T\left(P, P^{+}\right) R_{0}\left(P^{+}\right) \int d s P_{p}\left(P, P^{+}, s\right) \int d P^{\prime \prime} \int_{0}^{l} d \alpha B_{0}\left(\alpha, P^{+}, P^{\prime \prime}\right) \\
& x \sum_{n=0}^{r}\left(\begin{array}{l}
r \\
n
\end{array}\right) M_{n}\left(P^{\prime \prime}, t-t^{+}\right) s^{r-n}+\sum_{k=2}^{\infty} t^{+}<t d P^{+} T\left(P, P^{+}\right) S_{k}\left(P^{+}\right) \int d s p_{s k}\left(P, P^{+}, s\right) \\
& \times \int d P^{\prime \prime} B_{s k}\left(P^{+}, P^{\prime \prime}\right) \sum_{n_{1}=0}^{r}\left(\begin{array}{l}
r \\
n_{1}
\end{array}\right) M_{n_{1}}\left(P^{\prime \prime}, t-t^{+}\right) \cdots \sum_{n_{k}=0}^{r-n_{1} \cdots-n_{k-1}}\left(\begin{array}{c}
r-n_{1} \cdots \cdots-n_{k-1} \\
n_{k}
\end{array}\right) \\
& \times M_{n_{k}}\left(P^{\prime \prime}, t-t^{+}\right) s^{r-n_{1}} \cdots \cdots-n_{k}+t_{t}^{+} \int_{t} d P^{+} T\left(P, P^{+}\right) \int d P^{\prime} \sigma\left(P^{+*}, P^{\prime}\right) \int d s p_{1}\left(P^{\prime}, P^{\prime}, s\right) \\
& x \int d P^{\prime \prime} E\left(P^{\prime}, F^{\prime}\right) \int d P^{c} B\left(P^{\prime \prime}, P^{c}\right) \sum_{n=0}^{r}\left(r_{n}^{r}\right) M_{n}\left(P^{c}, t-t^{\prime}\right) s^{r-n}+\sum_{k=2}^{\infty} \int_{t}^{+}<t P^{+} \\
& \times T\left(P, P^{+}\right) \int d P^{\prime} \sigma\left(P^{+}, P^{\prime}\right) \int d S P_{k}\left(P, P^{\prime}, s\right) \int d P_{1} \cdots \int d P_{k} \varepsilon_{k}\left(P^{\prime}, P_{1}, \cdots, P_{k}\right) \\
& x \int d P_{1} c_{B}\left(P_{1}, P_{1}^{c}\right) \cdots \int d P_{k}^{c_{1}} B\left(P_{k}, P_{k}^{c}\right) \sum_{n_{1}=0}^{r}\left(\begin{array}{l}
r \\
n_{1}
\end{array}\right) M_{n_{1}}\left(P_{k}^{c}, t-t^{\prime}\right) \cdots \\
& \times \sum_{n_{k}=0}^{r-n_{1} \cdots-n_{k-1}}\left(\begin{array}{c}
r-n_{1} \cdots \cdots-n_{k-1} \\
n_{k}
\end{array}\right) M_{n_{k}}\left(P_{1}^{c}, t-t^{\prime}\right) s^{r-n_{1} \cdots \cdots-n_{k}}
\end{aligned}
$$

Recall $P\left(P, P^{\prime}, s\right)$ and $\psi(P, S, t)$ are probability functions so that

$$
\begin{aligned}
& \int P\left(P, P^{\prime}, s\right) s^{0} d s=1 \\
& M_{0}(P, t)=\int \psi(P, s, t) s^{0} d s=1
\end{aligned}
$$

Note in TERM 4 and TERMG that any one of the $n_{j}(j=1, \ldots, k)$ could be equal to $r$ and hence there are $k$ ways to get $M_{r}$ from these terms.

Collecting the $M_{r}$ terms

39 


$$
\begin{aligned}
& M_{r}(P, t)=\int_{t}^{+}<t d P^{+} T\left(P, P^{+}\right) R_{0}\left(P^{+}\right) \int d P^{\prime \prime} \int_{0}^{1} d \alpha B_{0}\left(\alpha, P^{+}, P^{\prime \prime}\right) M_{r}\left(P^{\prime \prime}, t-t^{+}\right) \\
& +\sum_{k=2}^{\infty} e^{+} \int_{t} d P^{+} T\left(P, P^{+}\right) S_{k}\left(P^{+}\right) \int d P^{\prime \prime} B_{s k}\left(P^{+}, P^{\prime \prime}\right) k M_{r}\left(P^{\prime \prime}, t-t^{+}\right) \\
& +\int_{t}^{+}<t P^{+} T\left(P^{+}, P^{+}\right) \int d P^{\prime} \sigma\left(P^{+}, P^{\prime}\right) \int d P^{\prime \prime} E\left(P^{\prime}, P^{\prime \prime}\right) \int d P^{c} B\left(P^{\prime \prime}, P^{c}\right) M_{r}\left(P^{c}, t-t^{\prime}\right) \\
& +\sum_{k=2}^{\infty} \int_{t}^{+}<t d P^{+} T\left(P, P^{+}\right) \int d P^{\prime} \sigma\left(P^{+}, P^{\prime}\right) \int d P_{1} \cdots \int d P_{k} \varepsilon_{k}\left(P^{\prime}, P_{1}, \cdots, P_{k}\right) \\
& x \int d P_{1} B\left(P_{1}, P_{1}^{c}\right) \cdots \int d P_{k}^{C_{B}} B\left(P_{k}, P_{k}^{c}\right) \sum_{j=1}^{k} M_{r}\left(P_{j}^{c}, t-t^{\prime}\right)+Q_{r}(P, t)
\end{aligned}
$$

where

$$
\begin{aligned}
& Q_{r}(P, t)=\int_{t}^{+}=t d P^{+} D\left(P, P^{+}, t\right) \int d s p d\left(P, P^{+}, s\right) s^{r}+t_{t}^{+}=t d P^{+} T\left(P, P^{+}\right) \int d P^{\prime} \sigma\left(P^{+}, P^{\prime}\right) \\
& x \int d s P_{0}\left(P, P^{\prime}, s\right) A\left(P^{\prime}\right) s^{r}+\int_{t}^{+}<t d P^{+} T\left(P, P^{+}\right) R_{0}\left(P^{+}\right) \int d s P_{p}\left(P, P^{+}, s\right) \\
& x \int d P^{\prime \prime} \int_{0}^{J} d \alpha B_{0}\left(\alpha, P^{+}, P^{\prime \prime}\right) \sum_{n=0}^{r-1}\left(\begin{array}{l}
r \\
n
\end{array}\right) M_{n}\left(P^{\prime \prime}, t-t^{\prime}\right) s^{r-n}+\sum_{k=2}^{\infty} t_{t}^{+}<t d P^{*} T\left(P_{s} P^{+}\right) S_{k}\left(P^{+}\right) \\
& x \int d s p_{s k}\left(P, P^{+}, s\right) \int d P^{\prime \prime} B_{s k}\left(P^{+}, P^{\prime \prime}\right) \sum_{n_{1}=0}^{r}\left(\begin{array}{l}
r \\
n_{1}
\end{array}\right) M_{n_{1}}\left(P^{\prime \prime}, t-t^{+}\right) \ldots \\
& \times \sum_{n_{k}=0}^{r-n_{1} \cdots \cdots-n_{k-1}}\left(\begin{array}{c}
r-n_{1} \cdots \cdots-n_{k-1} \\
n_{k}
\end{array}\right) M_{n_{k}}\left(p n, t-t^{+}\right)_{s}^{r-n_{1} \cdots \cdots-n_{k}} \prod_{i=1}^{k}\left(1-\delta_{n_{i}} r^{\prime}\right. \\
& +\int_{t}^{+}<t d P^{+} T\left(P^{\prime}, P^{+}\right) \int d P^{\prime}{ }_{\sigma}\left(P^{+}, P^{\prime}\right) \int d s P_{1}\left(P^{\prime}, P^{\prime}, s\right) \int d P^{\prime \prime} E\left(P^{\prime}, P^{\prime \prime}\right) \int d P^{C} B^{\prime}\left(P^{\prime \prime}, P^{C}\right) \\
& x \sum_{n=0}^{r-1}\left(C_{n}^{r}\right) M_{n}\left(P^{c}, t-t^{\prime}\right) s^{r-n}+\sum_{k=2}^{\infty} t^{+} \int_{<t} d P^{+} T\left(P^{\prime}, P^{+}\right) \int d P^{\prime} \sigma\left(P^{+}, P^{\prime}\right) \\
& \times \int d S P_{k}\left(P, P^{\prime}, s\right) \int d P_{1} \ldots \int d P_{k} \varepsilon_{k}\left(P^{\prime}, P_{1}, \ldots, P_{k}\right) \int d P_{1} B\left(P_{1}, P_{1} C_{1}\right) \ldots \int d P_{k}^{C_{B}}\left(P_{k}{ } P_{k} C_{k}\right)
\end{aligned}
$$

40 


$$
\begin{aligned}
& \times \sum_{n_{1}=0}^{r}\left(c_{n_{1}}^{r}\right) M_{n_{1}}\left(P_{k}^{c}, t-t^{\prime}\right) \sum_{n_{2}=0}^{r-n_{1}}\left(\begin{array}{c}
r-n_{1} \\
n_{2}
\end{array}\right) M_{n_{2}}\left(P_{k-1}^{c}, t-t^{\prime}\right) \cdots \\
& \times \sum_{n_{k}=0}^{r-n_{1}-\cdots-n_{k-1}}\left(\begin{array}{c}
r-n_{1}, \cdots-n_{k-1} \\
n_{k}
\end{array}\right) M_{n_{k}}\left(P_{1}^{c}, t-t^{\prime}\right) s^{r-n_{1}} \cdots-n_{k} \prod_{i=1}^{k}\left(1-\delta_{n_{j}} r^{\prime}\right)
\end{aligned}
$$

The first two moments are usually the only ones desired. The source term $Q_{1}$ for the $M_{1}$ equation and the source term $Q_{2}$ for the $M_{2}$ equation are given below. From eq. (109)

$$
\begin{aligned}
& Q_{1}(P, t)=\int_{t}^{+} \int_{=t} d P^{+} D\left(P, P^{+}, t\right) \int d s P_{d}\left(P, P^{+}, s\right) s+t_{t}^{+}<t d P^{+} T\left(P_{,} P^{+}\right) \int d P^{\prime} \sigma\left(P^{+}, P^{\prime}\right) \\
& x \int d s p_{0}\left(P, P^{\prime}, s\right) A\left(P^{\prime}\right) s+\sum_{k=2}^{\infty} \int_{t}^{+}<t d P^{+} T\left(P, P^{+}\right) s_{k}\left(P^{+}\right) \int d s p s k\left(P, P^{+}, s\right) s
\end{aligned}
$$

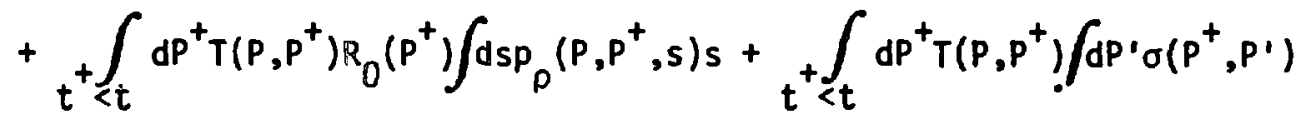

$$
\begin{aligned}
& x \int d s p_{7}\left(P^{\prime}, P^{\prime}, s\right) \int d P^{\prime \prime} E\left(P^{\prime}, P^{\prime \prime}\right) s+\sum_{k=2}^{\infty} t_{t}^{+} \int_{t} d P^{+} T\left(P^{\prime}, P^{+}\right) \int d P^{\prime} \sigma\left(P^{+}, P^{\prime}\right) \\
& x \int d s p_{k}\left(P, P^{\prime}, s\right) \int d P_{1} \cdots \int d P_{k} \varepsilon_{k}\left(P^{\prime}, P_{1}, \ldots, P_{k}\right) s
\end{aligned}
$$

For $r=2$ the splitting and multiplication terms in eq. (109) have a number of sums which must be evaluated. First consider the sum in the multiplication term in eq. (109) for $r=2$

$$
\begin{aligned}
& x_{m}=\sum_{n_{1}=0}^{2}\left({ }_{n_{1}}^{2}\right) M_{n_{1}}\left(p_{k}^{c}, t-t^{\prime}\right) \sum_{n_{2}=0}^{2-n_{1}}\left(\begin{array}{c}
2-n_{1} \\
n_{2}
\end{array}\right) M_{n_{2}}\left(p_{k-1}^{c}, t-t^{\prime}\right) \cdots \\
& x \sum_{n_{k}=0}^{2-n_{1} \cdots \cdots-n_{k-1}}\left(\begin{array}{c}
2-n_{1} \cdots \cdots-n_{k-1} \\
n_{k}
\end{array}\right) M_{n_{k}}\left(p_{1}^{c}, t-t^{\prime}\right) s^{r-n_{1} \cdots \cdots-n_{k}} \prod_{i=1}^{k}\left(1-\delta_{n_{i} 2}\right)
\end{aligned}
$$


Note by the sumation limits that

$0 \leq n_{1}+n_{2}+\cdots+n_{k} \leq 2$

and also that the $\left(1-\delta_{n_{i} 2}\right)$ factor requires $n_{i} \neq 2$ so that

$$
0 \leq n_{\mathbf{i}} \leq 1
$$

for $1 \leq i \leq k$. The terms in the sum (111) are separated below into $k+1$ mutually exclusive groups of terms which are evaluated and then summed to produce a simpler expression for $x_{m}$. The $j^{\text {th }}$ group $(j=1,2, \cdots, k)$ of terms consists of all terms for which $n_{1}=n_{2}=\cdots \cdot=n_{j-1}=0$ and $n_{j}=1$. The $k+1$ st group of terms consists of the single term for which $n_{1}=n_{2}=\cdots=n_{k}=0$. These groups are evaluated in the following paragraph.

If $n_{f}=1$ then by eq. (111A) either one or zero other

$n_{i}=1(i=2,3, \cdots, k)$, yielding

$\left({ }_{1}^{2}\right) M_{1}\left(P_{k}^{c}, t-t^{\prime}\right)\left[\sum_{j=1}^{k-1} M_{1}\left(P_{j}^{c}, t-t^{\prime}\right)+s\right]$

If $n_{1}=0$ and $n_{2}=1$ then either one or zero other $n_{i}=1 \quad(i=3,4, \cdots, k)$

$\left(\begin{array}{l}2 \\ 1\end{array}\right) M_{1}\left(p_{k-1}^{c}, t-t^{\prime}\right)\left[\sum_{j=1}^{k-2} M_{1}\left(p_{j}^{c}, t-t^{\prime}\right)+s\right]$

If $n_{1}=n_{2}=0$ and $n_{3}=1$ then either one or zero other $n_{i}=1 \quad(i=4,5, \ldots, k)$

$\left({ }_{1}^{2}\right) M_{1}\left(P_{k-2}^{c}, t-t^{\prime}\right)\left[\sum_{j=1}^{k-3} M_{1}\left(P_{j}^{c}, t-t^{\prime}\right)+s\right]$ 
If $n_{1}=n_{2}=\cdots=n_{k-2}=0$ and $n_{k-1}=1$ then either one or zero other

$n_{i}=r(i=k)$

$\left(\begin{array}{l}2 \\ 1\end{array} M_{1}\left(P_{2}^{c}, t-t^{\prime}\right)\left[\sum_{j=1}^{1} M_{1}\left(P_{j}^{c}, t-t^{\prime}\right)+s\right]\right.$

If $n_{1}=\cdots=n_{k-1}=0$ and $n_{k}=1$

$\left(\frac{2}{1}\right) M_{1}\left(P_{1}^{c}, t-t^{\prime}\right) s$

If $n_{\mathrm{l}}=\cdots=n_{k}=0$

$s^{2}$

Hence adding eqs. (111C)-(116)

$x_{m}=s^{2}+2 s \sum_{\ell=1}^{k} M_{1}\left(P_{l^{c}}^{c}, t-t^{\prime}\right)+2 \sum_{l=2}^{k} M_{1}\left(P_{l^{c}}^{c}, t-t^{\prime}\right)$

$x \sum_{j=1}^{\ell-1} M_{1}\left(P_{j}^{c}, t-t^{\prime}\right)$

Note that the term in eq. (109) involving the $k$ particles emerging from a splitting event has the same form as the term involving $k$ particles emerging from a collision except that in a splitting event all particles exit at P". Thus using eq. (117) with $P_{j}^{c}=p "$

$x_{s}=s^{2}+2 s k M_{1}\left(P^{\prime \prime}, t-t^{+}\right)+2 M_{1}^{2}\left(p^{\prime \prime}, t-t^{+}\right) \sum_{\ell=2}^{k} \sum_{j=1}^{\ell-1} 1$

$\sum_{\ell=2}^{k} \sum_{j=1}^{\ell-1} 1=\sum_{\ell=2}^{k}(\ell-1)=1+2+\ldots+k-1=\frac{k(k-1)}{2}$ 


$$
x_{s}=s^{2}+2 s k M_{1}\left(P^{\prime \prime}, t-t^{+}\right)+k(k-1) M_{1}^{2}\left(P^{\prime \prime}, t-t^{+}\right)
$$

From eqs. (109), (117), and (120)

$$
\begin{aligned}
& Q_{2}(P, t)=t_{t^{+}=t} d P^{+} D\left(P, P^{+}, t\right) \int d s P_{d}\left(P, P^{+}, s\right) s^{2}+t_{t}^{+} \int_{t} d P^{+} T\left(P, P^{+}\right) \\
& x \int d P^{\prime} \sigma\left(P^{+}, P^{\prime}\right) \int d s P_{0}\left(P^{\prime}, P^{\prime}, s\right) A\left(P^{\prime}\right) s^{2}+\int_{t}^{+}<t d P^{+} T\left(P, P^{+}\right) R_{0}\left(P^{+}\right) \\
& x \int d s p_{\rho}\left(P, P^{+}, s\right) \int d P^{\prime \prime} \int_{0}^{1} d \alpha B_{0}\left(\alpha, P^{+}, P^{\prime \prime}\right) \sum_{n=0}^{1}\left({ }_{n}^{2}\right) M_{n}\left(P^{\prime \prime}, \hat{t}-\hat{r}^{0}\right) s^{2-n} \\
& +\sum_{k=2}^{\infty} t_{t}^{+}<t d P^{+} T\left(P, P^{+}\right) s_{k}\left(P^{+}\right) \int d s p_{s k}\left(P, P^{+}, s\right) \int d P^{\prime \prime} B_{s k}\left(P^{+}, P^{\prime \prime}\right)\left\{s^{2}+\right. \\
& \left.2 s k M_{1}\left(P^{\prime \prime}, t-t^{+}\right)+k(k-1) M_{1}^{2}\left(P^{\prime \prime}, t-t^{+}\right)\right\}+\int_{t}^{+}<t P^{+} T\left(P, P^{+}\right) \\
& x \int d P^{\prime} \sigma\left(P^{+}, P^{\prime}\right) \int d s p_{1}\left(P_{0}, P^{\prime}, s\right) \int d P^{\prime \prime} E\left(P^{\prime}, P^{\prime \prime}\right) \int d P^{C} B\left(P^{\prime \prime}, P^{c}\right) \sum_{n=0}^{1}\left({ }_{n}^{2}\right) s^{2-n} \\
& x M_{n}\left(P^{c}, t-t^{\prime}\right)+\sum_{k=2}^{\infty} t^{+} \int_{<t} d P^{+} T\left(P^{+}, P^{+}\right) \int d P^{\prime} \sigma\left(P^{+}, P^{\prime}\right) \int d s P_{k}\left(P, P^{\prime}, s\right) \\
& x \int d P_{1} \cdots \int d P_{k} \varepsilon_{k}\left(P^{\prime}, P_{1}, \cdots, P_{k}\right) \int d P_{1} c_{B}\left(P_{1}, P_{1}^{c}\right) \cdots \iint_{d P_{k}} B_{\left(P_{k}, P_{k}^{c}\right)} \\
& x\left\{s^{2}+2 s \sum_{\ell=1}^{k} M_{l}\left(P_{\ell}^{c}, t-t^{\prime}\right)+2 \sum_{\ell=2}^{k} M_{l}\left(P_{\ell}^{c}, t-t^{\prime}\right) \sum_{j=1}^{\ell-1} M_{1}\left(P_{j}^{c}, t-t^{\prime}\right)\right\}
\end{aligned}
$$

44 
B) Choice of Non-Analog Kernels and Weight Multipliers.

By using non-analog kernels one tries to obtain the same $M_{1}$ as in the analog game but with a smaller variance. If there are no Russian Roulette or splitting events, i.e.

$$
R_{0}\left(P^{+}\right)=S_{k}\left(P^{+}\right)=0
$$

then it is easy, if somewhat tedious, to show how to pick kernels and weight multipliers which result in a game with the same $M_{1}$ as in the analog game. Substituting the definitions of Chapter II into eq. (108) results in

$$
\begin{aligned}
& M_{r}(R, w, t)=\int_{t}^{+} \int_{c t} d R^{+} d w^{+} T\left(R, R^{+}\right) \delta\left(w^{+}-w_{t} w\right) \int d R^{\prime} d w^{\prime} \sigma\left(R^{\prime}\right) \\
& x \delta\left(R^{\prime}-R^{+}\right) \delta\left(w^{\prime}-w_{\sigma^{\prime}} w^{+}\right) \int d R^{\prime \prime} d w^{\prime \prime} E\left(R^{\prime}, R^{\prime \prime}\right) \delta\left(w^{\prime \prime}-w_{\varepsilon_{1}} w^{\prime}\right) \int d R^{c} d w_{c} \\
& x \delta\left(R^{c}-R^{\prime \prime}\right)\left\{H\left(w^{\prime \prime}-w_{C O}\right) \delta\left(w_{c}-w^{\prime \prime}\right)+\left(1-H\left(w^{\prime \prime}-w_{c o}\right)\right)\right. \\
& \left.x\left[\frac{w^{\prime \prime}}{w_{e}} \delta\left(w_{c}-w_{e}\right)+\left(1-\frac{w^{\prime \prime}}{w_{e}}\right) \delta\left(w_{c}\right)\right]\right\} M_{r}\left(p^{c}, t-t^{\prime}\right) \\
& +\sum_{k=2}^{\infty} t_{t}^{+} \int_{<t} d R^{+} d w^{+} T\left(R, R^{+}\right) \delta\left(w^{+}-w_{t} w\right) \int d R^{\prime} d w^{\prime} \sigma\left(R^{\prime}\right) \delta\left(R^{\prime}-R^{+}\right) \\
& x \delta\left\langle w^{\prime}-w_{\sigma^{\prime}} w^{+}\right) \int d R_{1} d w_{1} \cdots \int d R_{k} d w_{k} \varepsilon_{k}\left(R^{\prime}, R_{1}, \cdots, R_{k}\right) \delta\left(w_{1}-w_{\varepsilon_{k}} w^{\prime}\right) \cdots \\
& \times \delta\left(w_{k}-w_{\varepsilon_{k}} w^{\prime}\right) \prod_{i=1}^{k} \int d R_{i}^{c} d w_{c_{i}} \delta\left(R_{i}^{c}-R_{i}\right)\left\{H\left(w_{i}-w_{c o}\right) \delta\left(w_{c_{i}}-w_{i}\right)\right. \\
& \left.+\left(1-H\left(w_{i}-w_{c o}\right)\right)\left[\frac{w_{i j}}{w_{e}} \delta\left(w_{c_{i}}-w_{e}\right)+\left(1-\frac{w_{i}}{w_{e}}\right) \delta\left(w_{c_{i}}\right)\right]\right\} \\
& x \sum_{j=1}^{k} M_{r}\left(R_{j}^{c}, w_{c_{j}}, t-t^{\prime}\right)+Q_{r}(P, t)
\end{aligned}
$$


Performing the integrations over $\delta$ functions and noting:

1) $w_{i}=w_{\varepsilon_{k}} w^{\prime}$ for all $i$, thus either $w_{i} \geq w_{c o}$ or $w_{i}<w_{c o}$ for all $i$ (123)

2) $M_{r}(R, n, t)=0$ since a particle of weight zero can contribute nothing to the score, hence $M_{r}\left(R, w \delta_{n, l}, t\right)=\delta_{n, l} M_{r}(R, w, t)$

3) $t^{+}$changes to $t^{\prime}$ when the integration of $\delta\left(R^{\prime}-R^{+}\right)$makes

$$
R^{\prime}=R^{+}
$$

one arrives at

$$
\begin{aligned}
& M_{r}(R, w, t)=\int_{t<t} d R^{\prime} T\left(R, R^{\prime}\right) \sigma\left(R^{\prime}\right) \int d R^{\prime \prime} E\left(R^{\prime}, R^{\prime \prime}\right)\left\{H \left(W_{t} w_{\sigma} W_{E_{1}}{ }^{-W} c 0^{\prime}\right.\right.
\end{aligned}
$$

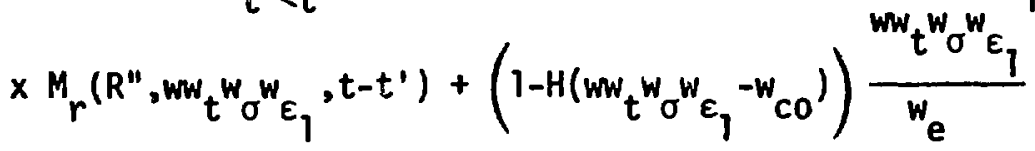

$$
\begin{aligned}
& \left.x M_{r}\left(R^{\prime \prime}, w_{e}, t-t^{\prime}\right)\right\}+\sum_{k=2}^{\infty} \int_{t^{\prime}<t} d R^{\prime} T\left(R, R^{\prime}\right) \sigma\left(R^{\prime}\right) \int d R_{1} \cdots \int d R_{k} \\
& x \varepsilon_{k}\left(R^{\prime}, R_{1}, \cdots, R_{k}\right)\left\{H\left(w w_{t} w_{\sigma} w_{\varepsilon_{k}}-w_{c o}\right) \sum_{j=1}^{k} M_{r}\left(R_{j}, w w_{t} w_{\sigma} w_{\varepsilon_{k}}, t-t^{\prime}\right)\right. \\
& +\left(1-H\left(w_{w^{\prime}} w_{\sigma} w_{\varepsilon_{k}}{ }^{-w_{c o}}\right)\right) \sum_{b_{1}=0}^{1} \cdots \sum_{b_{k}=0}^{1}\left(\frac{w_{t} w_{\sigma} w_{\varepsilon_{k}}}{w_{e}}\right)^{b_{1}+\cdots+b_{k}}\left(1-\frac{w_{t} w_{\sigma} w_{k}}{w_{e}}\right)^{k-b_{1} \cdots \cdots-b_{k}} \\
& \left.x \sum_{j=1}^{k} M_{r}\left(R_{j}, w e, t-t^{\prime}\right) \delta_{b_{j}, 1}\right\}+Q_{r}(R, w, t)
\end{aligned}
$$

The sums over the $b_{l}$ 's give all possible combinations for the survivals or deaths of the $k$ particles playing the weight cutoff game. Survival of the $e^{\text {th }}$ particle corresponds to $b_{\ell}=1$ and death corresponds to $b_{\ell}=0$. The $\delta_{b_{j}, l}$ in eq. (1248) allows only surviving 
particles to contribute.

Using eqs. (A16) and (A18) one arrives at

$$
\begin{aligned}
& M_{r}(R, w, t)=\int_{t^{\prime}<t} d R^{\prime} T\left(R, R^{\prime}\right) \sigma\left(R^{\prime}\right) \int d R^{\prime \prime} E\left(R^{\prime}, R^{\prime \prime}\right)\left\{H\left(W_{t} W_{\sigma} w_{\varepsilon} \varepsilon^{-W_{c}} c 0^{\prime}\right)\right.
\end{aligned}
$$

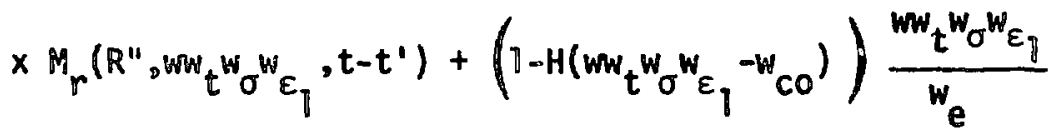

$$
\begin{aligned}
& \left.x M_{r}\left(\mathbb{R}^{\prime \prime},{ }^{*+e}, t-t^{0}\right)\right\}+\sum_{k=2}^{\infty} \int_{t^{0}<t} d R^{0} T\left(R, R^{0}\right) \sigma\left(R^{0}\right) \int d R_{1} \cdots \int d R_{k} \\
& x \varepsilon_{k}\left(R^{\prime}, R_{j}, \ldots, R_{k}\right)\left\{H\left(w w_{t} w_{\sigma} w_{\varepsilon_{k}}-w_{c o}\right) \sum_{j=1}^{k} M_{r}\left(R_{j}, w w_{t} w^{w w} \varepsilon_{k}, t-t^{\prime}\right)\right. \\
& \left.+\frac{w_{c} w_{\sigma}{ }^{W} \varepsilon_{k}}{w_{e}}\left(1-H\left(W w_{t} w_{\sigma} w_{\varepsilon_{k}}-w_{c o}\right)\right) \sum_{j=1}^{k} M_{r}\left(R_{j}, w_{e}, t-t^{\prime}\right)\right\} \\
& +Q_{p}\left(R_{0} w, t\right)
\end{aligned}
$$

Eq. (125) is of the form

$$
M_{r}(R, w, t)=K M_{r}(R, W, t)+Q_{r}(R, W, t)
$$

where the form of the operator $K$ can be inferred from eq. (125). A Neumann sequence may be generated using the iterøtive procedure (index i)

$$
M_{r}^{0}(R, w, t)=0
$$


and

$M_{r}^{i+1}(R, w, t)=K M_{r}^{i}(R, w, t)+Q_{r}(R, w, t)$.

With suitable restrictions on $K$ (see Appendix $C$ ) the Neumann

sequence converges to a unique solution. From here on, it will be ass'smed $K$ is such that there exists a unique solution to the $M_{p}$ equation. Now attempt to separate the weight dependence of $M_{r}$. Replace by aw in eq. (125) and then substitute

$M_{r}(R, a w, t)=a^{r} F_{r}(R, w, t)=a^{r} w F(R, \mathbb{r}, t)$

into eq. (125), divide the result by $\mathrm{a}^{r}$, and note that for a>0

$H(a x-b)$ equals $H(x-b / a)$, this yields

$F_{p}(R, w, t)=\int_{t^{\prime}<t} d R^{\prime} T\left(R, R^{\prime}\right) \sigma\left(R^{\prime}\right) \int d R^{\prime \prime} E\left(R^{\prime}, R^{\prime \prime}\right)\left\{H\left(w w_{t} w_{\sigma} w_{\varepsilon_{1}}-w_{c o} / a\right)\right.$

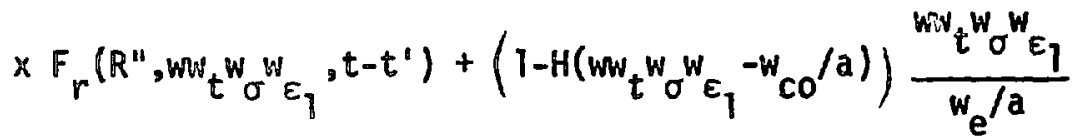

* $\left.F_{p}\left(R^{\prime \prime}, \frac{W_{e}}{a}, t-t^{\prime}\right)\right)+\sum_{k=2}^{\infty} \int_{t^{\prime}<t} d R^{\prime} T\left(R, R^{\prime}\right) \sigma\left(R^{\prime}\right) \int d R_{1} \cdots \int d R_{k}$

$x \varepsilon_{k}\left(R^{\prime}, R_{j}, \cdots, R_{k}\right)\left\{H\left(w w_{t} w_{\sigma} w_{\varepsilon_{1}}-w_{c o} / a\right) \sum_{j=1}^{k} F_{r}\left(R_{j}, w w_{t} w_{\sigma} w_{\varepsilon_{k}}, t-t^{\prime}\right)\right.$

$+\frac{w_{t} w^{W} \varepsilon_{k}}{w_{e} / a}\left(1-H\left(w_{t} w_{\sigma} w_{c_{1}}{ }^{\left.-w_{c o} / a\right)}\right) \sum_{j=1}^{k} F_{r}\left(R_{j} * \frac{w_{e}}{a}, t-t^{\prime}\right)\right\}$

$+Q_{r}(R, a w, t) a^{-r}$

If $w_{e}$ and $w_{c o}$ are proportional to the particle weight, that is:

$w_{e}(a w)=a w_{e}(w)$

(125F)

$w_{c o}(a w)=a w(w)$ 
and if $Q_{r}$ is proportional to the $r$ th power of the particle weight, that is

$Q_{r}(R, a w, t)=a^{r} Q_{r}(R, w, t)$

then eq. (125B) for $F_{r}$ is independent of "a" and identical to eq. (125) for $M_{r}$. Thus if eqs. $(125 F)-(125 H)$ hold the separation works and $F_{r}$ equals $M_{r}$, which implies

$M_{r}(R, w, t)=w^{r_{M}}(R, 1, t)$.

(Conditions (125F) and (125G) make the particle's history independent of its weight as in Chapter i)

When $r$ is equal to une, conditions (125F) and (125G) are not required. To see this, use eqs. (125D) and (125H) to set every weight argument in eq. (125E) to $w$ and then use $H(x)+(1-H(x))=1$ to obtain $F_{1}(R, W, t)=\int_{t^{\prime}<t} d R^{\prime} T\left(R, R^{\prime}\right) \sigma\left(R^{\prime}\right) \int d R^{\prime \prime} E\left(R^{\prime}, R^{\prime \prime}\right) W_{t} W \sigma{ } \varepsilon_{1} F_{1}\left(R, w, t-t^{\prime}\right)$

$+\sum_{k=2}^{\infty} \int_{t^{\prime}<t} d R^{\prime} T\left(R, R^{\prime}\right) \sigma\left(R^{\prime}\right) \int d R_{1} \cdots \int d R_{k} \varepsilon_{k}\left(R^{\prime}, R_{1}, \cdots, R_{k}\right) W_{t}{ }^{W} \sigma^{W} \varepsilon_{k}$ $x \sum_{j=1}^{k} F_{1}\left(R_{j}, w, t-t^{\prime}\right)+Q_{j}(R, w, t)$.

Note that $F_{1}$ is independent of $w_{e}$ and $w_{c o}$ so eq. (i25H) is the only requirement for

$H_{1}(R, w, t)=W M_{7}(R, l, t)$

From eq. (110) and the definitions in Chapter II 


$$
\begin{aligned}
& Q_{1}(R, w, t)=t_{t}^{+}=t d R^{+} d w^{+} T\left(R, R^{+}\right) \delta\left(w^{+}-w w_{t}\right) \delta\left(\left|\overrightarrow{r^{+}-\vec{r}}\right|-v t\right) \\
& x \int d s p_{d}\left(P, R^{+}, w^{+}, s\right) s+\int_{t}^{+}<t d R^{+} d w^{+} T\left(R, R^{+}\right) \delta\left(w^{+}-w w_{t}\right) \\
& x \int d R^{\prime} d w^{\prime} \sigma\left(R^{\prime}\right) \delta\left(R^{\prime}-R^{+}\right) \delta\left(W^{\prime}-w_{\sigma} w^{+}\right) \int d s P_{0}\left(P, R^{\prime}, w^{\prime}, s\right) A\left(R^{\prime}\right) s \\
& +\int_{t}^{+} \int_{<t} d R^{+} d w^{+} T\left(R, R^{+}\right) \delta\left(w^{+}-w w_{t}\right) \int d R^{\prime} d w^{0} \sigma\left(R^{\prime}\right) \delta\left(R^{\prime}-R^{+}\right) \delta\left(w^{\prime}-w^{+} w_{\sigma}\right) \\
& \times \int d s p_{1}\left(P, R^{\prime}, w^{\prime}, s\right) s \int d R^{\prime \prime} d w^{\prime \prime} E\left(R^{\prime}, R^{\prime \prime}\right) \delta\left(w^{\prime \prime}-w_{\varepsilon_{1}} w^{\prime}\right) \\
& +\sum_{k=2}^{\infty} t^{+}<t d R^{+} d w^{+} T\left(R, R^{+}\right) \delta\left(w^{+}-w w_{t}\right) \int d R^{\prime} d w^{\prime} \sigma\left(R^{\prime}\right) \delta\left(R^{\prime}-R^{+}\right) \\
& x \delta\left(w^{\prime}-w_{\sigma^{\prime}} w^{+}\right) \int d s p_{k}\left(P, R^{\prime}, w^{\prime}, s\right) s \int d R_{1} d w_{1} \cdots \int d R_{k} d w_{k} \\
& x \varepsilon_{k}\left(R^{\prime}, R_{1}, \cdots, R_{k}\right) \delta\left(w_{1}-w_{\varepsilon_{k}} w^{\prime}\right) \cdots \delta\left(w_{k}-w_{\varepsilon_{k}} w^{\prime}\right)
\end{aligned}
$$

Performing the integrations over the delta functions and using

(123) and (124A)

$$
\begin{aligned}
& Q_{1}(R, w, t)=t_{t}^{+}=t d R^{+} T\left(R, R^{+}\right) \delta\left(\left|\vec{r}^{+}-\vec{r}\right|-v t\right) \int d s p_{d}\left(P, R^{+}, w w_{t}, s\right) s \\
& +\int_{t^{\prime}<t} d R^{\prime} T\left(R, R^{\prime}\right) \sigma\left(R^{\prime}\right) A\left(R^{\prime}\right) \int d s P_{0}\left(P, R^{\prime}, w w_{t} w_{\sigma}, s\right) s \\
& +\int_{t} \int_{t} d R^{\prime} T\left(R, R^{\prime}\right) \sigma\left(R^{\prime}\right) \int d s p_{j}\left(P, R^{\prime}, w_{t} w_{\sigma}, s\right) s \int d R^{\prime \prime} E\left(R^{\prime}, R^{\prime \prime}\right) \\
& +\sum_{k=2}^{\infty} \int_{t^{\prime}<t} d R^{\prime} T\left(R, R^{\prime}\right) \sigma\left(R^{\prime}\right) \int d s p_{k}\left(P, R^{\prime}, w w_{t} w_{\sigma}, s\right) s f R_{j} \cdots \int d R_{k} \varepsilon_{k}\left(R^{\prime}, R_{1}, \ldots, R_{k}\right)
\end{aligned}
$$

By eqs. (84) and (i27) $Q_{1}$ must be proportional to $w$. Thus, by eqs. (125H) and (125K)

$M_{1}\left(R, w w_{0}, t\right)=W_{1}\left(R, w_{0}, t\right)$

50 
From eq. (121) and the definitions of Chapter II

$$
\begin{aligned}
& Q_{2}(R, w, t)={ }_{t}^{+} \int_{=t} d R^{+} d w^{+} T\left(R, R^{+}\right) \delta\left(w^{+}-w w_{t}\right) \delta\left(||_{r}^{+}-\vec{r} \mid-v t\right) \\
& \times \int d s P_{d}\left(P, R^{+}, w^{+}, s\right) s^{2}+t_{t}^{+}<t d R^{+} d w^{+} T\left(R, R^{+}\right) \delta\left(w^{+}-w w_{t}\right) \\
& \times \int d R^{\prime} d w^{\prime} \sigma\left(R^{\prime}\right) \delta\left(R^{\prime}-R^{+}\right) \delta\left(w^{\prime}-w^{+} w_{\sigma}\right) \int d S P_{0}\left(P, R^{\prime}, w^{\prime}, s\right) s^{2} A\left(R^{\prime}\right) \\
& +t_{t}^{+}<t R^{+} d w^{+} T\left(R, R^{+}\right) \delta\left(w^{+}-w_{t}\right) \int d R^{\prime} d w^{\prime} \sigma\left(R^{\prime}\right) \delta\left(R^{\prime}-R^{+}\right) \delta\left(w^{\prime}-w^{+} w_{\sigma}\right) \\
& \times \int d S p_{1}\left(P, R^{\prime}, W^{\prime}, s\right) \int d R^{\prime \prime} d W^{\prime \prime} E\left(R^{\prime}, R^{\prime \prime}\right) \delta\left(W^{\prime \prime}-w_{\varepsilon_{1}} W^{\prime}\right) \int d R^{c} d W_{c} \delta\left(R^{c}-R^{\prime \prime}\right) \\
& \times\left\{H\left(w^{\prime \prime}-w_{c 0}\right) \delta\left(w_{c}-w^{\prime \prime}\right)+\left(1-H\left(w^{\prime \prime}-w_{c o}\right)\right)\left[\frac{w^{\prime \prime}}{w_{e}} \delta\left(w_{c}-w_{e}\right)\right.\right. \\
& \left.\left.+\left(1-\frac{w^{\prime \prime}}{w_{e}}\right) \delta\left(w_{c}\right)\right]\right\} \sum_{n=0}^{l}\left(i_{n}^{2}\right) M_{n}\left(R^{c}, w_{c}, t-t^{\prime}\right) s^{2-n} \\
& +\sum_{k=2}^{\infty} t_{t}^{+}<t R^{+} d w^{+} T\left(R, R^{+}\right) \delta\left(W^{+}-W W_{t}\right) \int d R^{\prime} d W^{\prime} \sigma\left(R^{\prime}\right) \delta\left(R^{\prime}-R^{+}\right) \\
& x \delta\left(w^{\prime}-w^{+} w_{\sigma}\right) \int d s p_{k}\left(P, R^{\prime}, w^{\prime}, s\right) \int d R_{1} d w_{1} \cdots \int d R_{k} d w_{k} \varepsilon_{k}\left(R^{\prime}, R_{1}, \cdots, R_{k}\right) \\
& x \delta\left(w_{1}-w_{\varepsilon_{k}} w^{\prime}\right) \cdots \delta\left(w_{k}-w_{\varepsilon_{k}} w^{\prime}\right) \prod_{i=1}^{k} \int d R_{i}^{c} d w_{c_{i}} \delta\left(R_{i}^{c}-R_{i}\right) \\
& \times\left\{H\left(w_{i}-w_{c o}\right) \delta\left(w_{c_{i}}-w_{i}\right)+\left(1-H\left(w_{i}-w_{c o}\right)\right)\left[\frac{w_{i}}{w_{e}} \delta\left(w_{c_{i}}-w_{e}\right)\right.\right. \\
& \left.\left.+\left(1-\frac{w_{i}}{w_{e}}\right) \delta\left(w_{c_{i}}\right)\right]\right\}\left\{s^{2}+2 s \sum_{\ell=1}^{k} M_{1}\left(R_{\ell}^{c}, w_{c_{\ell}}, t-t^{\prime}\right)\right. \\
& \left.+2 \sum_{\ell=2}^{k} M_{1}\left(R_{\ell^{c}}^{c} w_{c_{l}}, t-t^{\prime}\right) \sum_{j=1}^{\ell-1} M_{i}\left(R_{j}^{c}, w_{c_{j}}, t-t^{\prime}\right)\right\}
\end{aligned}
$$

51 
Integrating over the delta functions and noting (123)-(124A)

$$
\begin{aligned}
& Q_{2}(R, w, t)=\int_{t=t} d R^{+} T\left(R, R^{+}\right) \delta(|\vec{r}-\vec{r}|-v t) \int d s P_{d}\left(P, R^{+}, w w_{t}, s\right) s^{2} \\
& +\int_{t<t} d R^{\prime} T\left(R, R^{\prime}\right) \sigma\left(R^{\prime}\right) A\left(R^{\prime}\right) \int d S P_{0}\left(P, R^{\prime}, W w_{t} W_{\sigma}, s\right) s^{2} \\
& +\int_{t}<t R^{\prime} T\left(R, R^{\prime}\right) \sigma\left(R^{\prime}\right) \int d s p_{1}\left(P, R^{\prime}, W w_{t} W_{\sigma}, s\right) \int d R^{\prime \prime} E\left(R^{\prime}, R^{\prime \prime}\right) \\
& x\left\{H\left(w_{w^{\prime}} w_{\sigma} w_{\varepsilon_{1}}-w_{c}\right) \sum_{n=0}^{1}\left(\begin{array}{l}
2 \\
n
\end{array}\right) M_{n}\left(R^{\prime \prime}{ }^{\prime} w_{t^{\prime}} w_{\sigma} w_{\varepsilon_{1}}, t-t^{\prime}\right) s^{2-n}\right. \\
& \left.+\left(1-H\left(w_{t} w_{0} w_{\varepsilon_{1}}-w_{c o}\right)\right)\left(s^{2}+2 s \frac{w_{t} w_{\sigma} w_{1}}{w_{e}} M_{1}\left(R^{\prime \prime}, w_{e}, t-t^{\prime}\right)\right)\right\} \\
& +\sum_{k=2}^{\infty} \int_{t} d R^{\prime} T\left(R_{2}, R^{\prime}\right) \sigma\left(R^{\prime}\right) \int d s P_{k}\left(P, R^{\prime}, W_{t} W_{\sigma}, s\right) \int d R_{1} \cdots \iint d R_{k} \\
& x \varepsilon_{k}\left(R^{\prime}, R_{l}, \cdots, R_{k}\right)\left[H ( W w _ { t } w _ { \sigma } w _ { \varepsilon _ { k } } { } ^ { - W } c 0 ^ { \prime } ) \left\{s^{2}+2 s \sum_{\ell=1}^{k} M_{l}\left(R_{l}, w_{t} w_{\sigma} w_{\varepsilon_{k}}, t-t^{\prime}\right)\right.\right. \\
& \left.+2 \sum_{\ell=2}^{k} M_{1}\left(R_{\ell}, w^{w} w^{w} \sigma^{w} \varepsilon_{k}, t-t^{\prime}\right) \sum_{j=1}^{\ell-1} M_{1}\left(R_{j}, w^{w} t^{w} \sigma^{W} \varepsilon_{k}, t-t^{\prime}\right)\right\} \\
& +\left(1-H\left(w w_{t} w_{\sigma} w_{\varepsilon_{k}}{ }^{-w_{c o}}\right)\right) \sum_{b_{j}=0}^{1} \cdots \sum_{b_{k}=0}^{l}\left(\frac{w^{w} w^{w} w^{w} \varepsilon_{k}}{w_{e}}\right)^{b_{1}+\cdots+b_{k}} \\
& x\left(1-\frac{w_{w_{1}} w_{0} w_{k}}{w_{e}}\right)^{k-b_{1} \cdots \cdots-b_{k}}\left\{s^{2}+2 s \sum_{\ell=1}^{k} M_{1}\left(R_{\ell}, w_{e}, t-t^{\prime}\right) \delta_{b_{\ell}, 1}\right. \\
& \left.\left.+2 \sum_{\ell=2}^{k} M_{1}\left(R_{\ell}, w_{e}, t-t^{\prime}\right) \delta_{b_{\ell}, 1} \sum_{j=1}^{\ell-1} M_{1}\left(R_{j}, w_{e}, t-t^{\prime}\right) \delta_{b_{j}, 1}\right\}\right]
\end{aligned}
$$


Combining the scattering and multiplication terms and using eds. (A12)(A21)

$$
\begin{aligned}
& \left.Q_{L}, R, w, t\right)=\int_{t^{+}=t} d R^{+} T\left(R, R^{+}\right) \delta(|\vec{r}-\vec{r}|-v t) \int d s p_{d}\left(P, R^{+}, w w, s\right) s^{2} \\
& +\int_{t<t} d R^{0} T\left(R, R^{\prime}\right) \sigma\left(R^{\prime}\right) A\left(R^{\prime}\right) \int d s P_{0}\left(P, R^{\prime}, W W_{t} W^{W}, s\right) s^{2} \\
& +\sum_{k=1}^{\infty} \int_{t^{\prime}<t} d R^{\prime} T\left(R, R^{\prime}\right) \sigma\left(R^{\prime}\right) \int d s P_{k}\left(P, R^{\prime}, w w_{t} w_{\sigma}, s\right) \int d R_{1} \cdots d R_{k} \\
& x \varepsilon_{k}\left(R^{\prime}, R_{1}, \cdots, R_{k}\right)\left[H ( w w _ { t } w _ { \sigma } w _ { \varepsilon _ { k } } - w _ { c o } ) \left\{s^{2}+2 s \sum_{l=1}^{k} M_{1}\left(R_{\ell},{ }^{\prime} w_{t} w_{\sigma} w_{\varepsilon_{k}}, t-t^{\prime}\right)\right.\right. \\
& \left.+2 \sum_{\ell=2}^{k} M_{1}\left(R_{\ell}, w_{t^{\prime}} w^{\prime} w_{\varepsilon_{k}}, t-t^{\prime}\right) \sum_{j=1}^{\ell-1} M_{1}\left(R_{j}, w w_{t} w^{w} w_{\varepsilon_{k}}, t-t^{\prime}\right)\right\} \\
& +\left(1-H\left(w w_{t} t^{w} \sigma^{w} \varepsilon_{k}-w_{c o}\right)\right)\left\{s^{2}+2 s \frac{w_{t}^{w} \sigma^{w} E_{k}}{w_{e}} \sum_{\ell=1}^{k} M_{l}\left(R_{\ell}, w_{e}, t-t^{\prime}\right)\right. \\
& \left.\left.+2\left(\frac{t^{w} w^{w} \varepsilon_{k}}{w_{e}}\right)^{2} \sum_{l=2}^{k} M_{l}\left(R_{\ell}, w_{e}, t-t^{\prime}\right) \sum_{j=1}^{\ell-1} M_{l}\left(R_{j}, w_{e}, t-t^{\prime}\right)\right\}\right]
\end{aligned}
$$

53 
Using eq. (128), and noting $H(x)+(1-H(x))=1$

$$
\begin{aligned}
& Q_{2}(R, w, t)=\int_{t^{+}=t} d R^{+} T\left(R, R^{+}\right) \delta(|\vec{r}-\vec{r}|-v t) \int d s p_{d}\left(P, R^{+}, w w w_{t}, s\right) s^{2} \\
& +\int_{t^{\prime}<t} d R^{\prime} T\left(R, R^{\prime}\right) \sigma\left(R^{\prime}\right) A\left(R^{\prime}\right) \int d s P_{0}\left(P, R^{\prime}, w w_{t} w_{\sigma}, s\right) s^{2} \\
& +\sum_{k=1}^{\infty} \int_{t^{\prime}<t} d R^{\prime} T\left(R, R^{\prime}\right) \sigma\left(R^{\prime}\right) \int d s P_{k}\left(P, R^{\prime}, w w_{t} W_{\sigma^{\prime}}, s\right) \int d R_{1} \cdots \int d R_{k} \\
& x \varepsilon_{k}\left(R^{\prime}, R_{1}, \cdots, R_{k}\right)\left\{s^{2}+2 s \sum_{\ell=1}^{k} M_{1}\left(R_{j}, w w_{t} w_{\sigma} w_{\varepsilon_{k}}, t-t^{\prime}\right)\right. \\
& \left.+2 \sum_{\ell=2}^{k} M_{1}\left(R_{\ell}, w w_{t} w_{\sigma} w_{k}, t-t^{\prime}\right) \sum_{j=1}^{\ell-1} M_{1}\left(R_{j}, w w_{t} w_{\sigma} w_{\varepsilon_{k}}, t-t^{\prime}\right)\right\}
\end{aligned}
$$

Note that $Q_{1}$ and $Q_{2}$ (eqs. (127) and (130B)) are independent of the weight cutoff game. By eqs. (84), (128), and (130B) $Q_{2}$ must be proportional to $w^{2}$. Thus if the exit weight and cutoff weight are chosen as in eqs. (125F)-(125G) then eq. (125I) with $r=2$ implies

$$
M_{2}\left(R, w w_{0}, t\right)=w^{2} M_{2}\left(R, w_{0}, t\right)
$$


Consider eq. (125) for $M_{1}$. Let * indicate analog functions.

For an analog game eq. (127) with $w_{t}=w_{\sigma}=w_{\varepsilon_{1}}=w_{\varepsilon_{k}}=1$ and $w_{c o}=0$ becomes

$$
\begin{aligned}
& Q_{j}^{*}(R, w, t)=t_{t}^{+} \int_{=t} d R^{+} T^{*}\left(R, R^{+}\right) \delta\left(\left|\vec{r}^{+}-\vec{r}\right|-v t\right) \int d s p_{d}^{*}\left(P, R^{+}, w, s\right) s \\
& +\int_{t<t} d R^{\prime} T^{\star}\left(R, R^{\prime}\right) \sigma^{*}\left(R^{\prime}\right) A^{\star}\left(R^{\prime}\right) \int d S P_{0}^{*}\left(P, R^{\prime}, W, s\right) s \\
& +\int_{t} \int_{t} d R^{\prime} T^{\star}\left(R, R^{\prime}\right) \sigma^{\star}\left(R^{\prime}\right) \int d s P_{j}^{*}\left(P, R^{\prime}, W, s\right) s \int d R^{\prime \prime} E^{\star}\left(R^{\prime}, R^{\prime \prime}\right) \\
& +\sum_{k=2}^{\infty} \int_{t^{\prime}<t} d R^{\prime} T^{*}\left(R, R^{\prime}\right) \sigma^{*}\left(R^{\prime}\right) \int d s P_{k}^{*}\left(P, R^{\prime}, W, s\right) s \int d R_{1} \cdots \int d R_{k} E_{k}^{*}\left(R^{\prime}, R_{1}, \cdots, R_{k}\right)
\end{aligned}
$$

Now consider a non-analog game with weight multipliers:

$$
\begin{aligned}
& w_{t}=T^{\star}\left(R, R^{\prime}\right) / T\left(R, R^{\prime}\right) \\
& w_{\sigma}=\sigma^{*}\left(R^{\prime}\right) / \sigma\left(R^{\prime}\right) \\
& w_{\varepsilon_{j}}=E^{\star}\left(R^{\prime}, R^{\prime \prime}\right) / E\left(R^{\prime}, R^{\prime \prime}\right) \\
& w_{\varepsilon_{k}}=\varepsilon_{k}^{*}\left(R^{\prime}, R_{1}, \cdots, R_{k}\right) / \varepsilon_{k}\left(R^{\prime}, R_{1}, \cdots, R_{k}\right)
\end{aligned}
$$

Using eqs. (133),(127), and (84) the equation for $Q_{1}$ becomes:

$$
\begin{aligned}
& Q_{1}(R, w, t)=\int_{t=t}^{+} d R^{+} T\left(R, R^{+}\right) \delta(|\vec{r}-\vec{r}|-v t) \frac{T^{*}\left(R, R^{+}\right)}{T\left(R, R^{+}\right)} \int d s p_{d}\left(P, R^{+}, w, s\right) s \\
& +\int_{t} d R^{\prime} T\left(R, R^{\prime}\right) \sigma\left(R^{\prime}\right) \frac{T^{\star}\left(R, R^{\prime}\right)}{T\left(R, R^{\prime}\right)} \frac{\sigma^{*}\left(R^{\prime}\right)}{\sigma\left(R^{\prime}\right)} A\left(R^{\prime}\right) / d s P_{0}\left(P, R^{\prime}, H, s\right) s \\
& +\int_{t^{\prime}<t} d R^{\prime} T\left(R, R^{\prime}\right) \sigma\left(R^{\prime}\right) \frac{T^{\star}\left(R, R^{\prime}\right)}{T\left(R, R^{\prime}\right)} \frac{\sigma^{*}\left(R^{\prime}\right)}{\sigma\left(R^{\prime}\right)} \int d s p_{1}\left(P, R^{\prime}, W, s\right) s \int d R^{\prime \prime} E\left(R^{\prime}, R^{\prime \prime}\right) \\
& +\sum_{k=2}^{\infty} \int_{t^{\prime}<t} d R^{\prime} T\left(R, R^{\prime}\right) \sigma\left(R^{\prime}\right) \frac{T *\left(R, R^{\prime}\right)}{T\left(R, R^{\prime}\right)} \frac{\sigma^{*}\left(R^{\prime}\right)}{\sigma\left(R^{\prime}\right)} \int d s p_{k}\left(P, R^{\prime}, W, s\right) s \\
& x \int d R_{1} \cdots \int d R_{k} \varepsilon_{k}\left(R^{\prime}, R_{1}, \cdots, R_{k}\right)
\end{aligned}
$$


Let

$$
\left.\begin{array}{l}
A_{0}\left(R^{\prime}\right)=A\left(R^{\prime}\right) \\
A_{j}\left(R^{\prime}\right)=\int d R^{\prime \prime} E\left(R^{\prime}, R^{\prime \prime}\right) \\
A_{k}\left(R^{\prime}\right)=\int d R_{1} \cdots \int d R_{k} \varepsilon_{k}\left(R^{\prime}, R_{1}, \cdots, R_{k}\right) \quad k \geq 2
\end{array}\right\}
$$

If

$P_{d}\left(P, R^{+}, w, s\right)=P_{d}^{*}\left(P, R^{+}, w, s\right)$, and

$\sum_{k=0}^{\infty} A_{k}\left(R^{\prime}\right) P_{k}\left(P, R^{\prime}, w, s\right)=\sum_{k=0}^{\infty} A_{k}^{*}\left(R^{\prime}\right) P_{k}^{*}\left(P, R^{\prime}, w, s\right)$, then

$Q_{1}(R, w, t)=Q_{1}^{*}(R, w, t)$.

For an analog game eq. (125) with $w_{t}=w_{\sigma}=w_{\varepsilon_{1}}=w_{\varepsilon_{k}}=1$ and $w_{c o}=0$ becomes

$$
\begin{aligned}
& M_{l}^{*}(R, w, t)=\int_{t^{\prime}<t} d R^{\prime} T^{*}\left(R, R^{\prime}\right) \sigma^{\star}\left(R^{\prime}\right) \int d R^{\prime \prime} E^{\star}\left(R^{\prime}, R^{\prime \prime}\right) M_{j}^{*}\left(R^{\prime \prime}, W, t-t^{\prime}\right) \\
& +\sum_{k=2}^{\infty} \int_{t^{\prime}<t} d R^{\prime} T^{\star}\left(R, R^{\prime}\right) \sigma^{\star}\left(R^{\prime}\right) \int d R_{j} \cdots \int d R_{k} \varepsilon_{k}\left(R^{\prime}, R_{j}, \cdots, R_{k}\right) \sum_{j=1}^{k} M_{l}^{*}\left(R_{j}, W_{0}, t-t^{\prime}\right) \\
& +Q_{l}^{*}(R, w, t)
\end{aligned}
$$

For the non-analog case eq. (125) becomes (using eqs. (128) and (733) )

$$
\begin{aligned}
& M_{j}(R, w, t)=\int_{t,<t} d R^{\prime} T\left(R, R^{\prime}\right) \sigma\left(R^{\prime}\right) \int d R^{\prime \prime} E\left(R^{\prime}, R^{\prime \prime}\right)\left\{H\left(W w_{t} w^{w} \sigma^{w} \varepsilon_{q}{ }^{-w^{\prime}} c 0^{\prime}\right)\right. \\
& \times \frac{T^{*}\left(R, R^{\prime}\right)}{T\left(R, R^{\prime}\right)} \frac{\sigma^{*}\left(R^{\prime}\right)}{\sigma\left(R^{\prime}\right)} \frac{E^{*}\left(R^{\prime}, R^{\prime \prime}\right)}{E\left(R^{\prime}, R^{\prime \prime}\right)} M_{1}\left(R^{\prime \prime}, w, t-t^{0}\right)+\left(1-H\left(w w_{t}{ }^{w} \sigma^{w} \varepsilon_{p}-w_{c o}\right)\right) \\
& \left.x \frac{T^{*}\left(R, R^{\prime}\right)}{T\left(R, R^{\prime}\right)} \frac{\sigma^{*}\left(R^{\prime}\right)}{\sigma\left(R^{\prime}\right)} \frac{E^{*}\left(R^{\prime}, R^{\prime \prime}\right)}{E\left(R^{\prime}, R^{\prime \prime}\right)} M_{1}\left(R^{\prime \prime}, w, t-t^{\prime}\right)\right\}+\sum_{k=2}^{\infty} \int_{t} \int_{<t} d R^{\prime} T\left(R, R^{\prime}\right) \sigma\left(R^{\prime}\right) \\
& x \int d R_{1} \cdots \int d_{R_{k}} \varepsilon_{k}\left(R^{\prime}, R_{j}, \cdots, R_{k}\right)\left\{H\left(W w_{t}{ }^{W} \sigma^{w} \varepsilon_{k}-w_{c o}\right) \frac{T\left(R, R^{\prime}\right)}{T\left(R, R^{\prime}\right)} \frac{\sigma^{*}\left(R^{\prime}\right)}{\sigma\left(R^{\prime}\right)}\right.
\end{aligned}
$$

56 


$$
\begin{aligned}
& x \frac{\varepsilon_{k}^{*}\left(R^{\prime}, R_{1}, \cdots, R_{k}\right)}{\varepsilon_{k}\left(R^{0}, R_{1}, \cdots, R_{k}\right)} \sum_{j=1}^{k} M_{1}\left(R_{j}, w, t-t^{\prime}\right)+\left(1-H\left(m N_{t} W_{a} w_{\varepsilon_{k}}-w_{c 0}\right)\right) \frac{T *\left(R, R^{\prime}\right)}{T\left(R, R^{\prime}\right)} \\
& \left.\times \frac{\sigma^{*}\left(R^{\prime}\right)}{\sigma\left(R^{\prime}\right)} \frac{\varepsilon_{k}^{*}\left(R^{\prime}, R_{1}, \cdots, R_{k}\right)}{\varepsilon_{k}\left(R^{\prime}, R_{1}, \cdots, R_{k}\right)} \sum_{j=1}^{k} M_{1}\left(R_{j}, w, t-t^{\prime}\right)\right\}+Q_{1}(R, w, t)
\end{aligned}
$$

Using $H(x)+(1-H(x))=1$ eq. (140) becomes

$M_{1}(R, w, t)=\int_{t^{\prime}<t} d R^{\prime} T^{\star}\left(R, R^{\prime}\right) \sigma^{\star}\left(R^{\prime}\right) \int d R^{\prime \prime} E^{\star}\left(R^{\prime}, R^{\prime \prime}\right) M_{1}\left(R^{\prime \prime}, w, t-t^{\prime}\right)$

$+\sum_{k=2}^{\infty} \int_{t^{\prime}<t} d R^{\prime} T^{*}\left(R, R^{\prime}\right) \sigma^{*}\left(R^{\prime}\right) \int d R_{1} \cdots \int d R_{k} \varepsilon_{k}^{*}\left(R^{\prime}, R_{1}, \cdots, R_{k}\right) \sum_{j=1}^{k} M_{1}\left(R_{j}, w, t-t^{\prime}\right)$

$$
+Q_{1}(R, W, t)
$$

Since $Q_{1}=Q_{1}^{*}$ this is exactly the same equation that $M_{1}^{*}$ satisfies; hence if eqs. (133), (136), and (137) are satisfied,

$M_{1}(R, W, t)=M_{1}^{\star}(R, w, t)$

This shows it is possible to play a non-analog game whose mean score is the same as the mean score for the analog game. In general $M_{2} \neq M_{2}^{*}$, which implies the variances are different; several examples of this will be given in Chapter IV. 
Chapter IV. Examples

In IV-A two different estimators for flux in a region are compared. Using the equations of Chapter III it is shown (for an arbitrary medium) that both estimators give the same mean but different variances. The equations of Chapter III are then specialized in IV-B to one-speed infinite medium problems, and an analytic solution method is provided to solve these specialized equations exactly. The equations of IV-B are then used in IV-C to predict the variances in four different techniques for obtaining the expected number of collisions. Finally, in IV-D the sample variances in Monte Carlo calculations are compared with the predicted variances for each of the four techniques in IV-C.

\section{A. Collision and Track Length Estimators Compared}

Consider the problem of obtaining the integral of the flux over a given region $\Gamma$ in $(\vec{r}, \vec{v})$ space. Two estimators can be used to solve this problem; the collision estimator scores $1 / \sigma$ every time a colfision occurs in $\Gamma$ and the track length estimator scores the distance (track length) a neutron travels through $\Gamma$ between events. It is shown below, first on physical grounds and then using eqs. (125) and (127), that these estimators result in the same expected value for the flux but: different variances.

\section{Collision Estimator}

The number of collisions per unit volume at energy $E$ is equal to the flux $\phi(\vec{r}, E)$ multiplied by the macroscopic cross section $\sigma(\vec{r}, E)$, or 
$d N(\vec{r}, E)=\sigma(\vec{r}, E) \phi(\vec{r}, E) d V$

$\phi(\vec{r}, E) d V=d N(\vec{r}, E) / \sigma(\vec{r}, E)$

Each collision in $d V$ contributes $1 / \sigma$ to the flux in the volume $d V$. Thus an estimate of the integral (over space, energy, and time) of the flux in region $\Gamma$ may be obtained by scoring $1 / \sigma(\vec{r}, E)$ at each collision occurring in the region in the given time interval.

\section{Track Length Estimator}

The significance of this estimator follows from the property that flux at a given errergy equals speed times particle number density. 6 Thus, the integral of flux over a given region at a given time equals speed times the number of neutrons then in that region. For each neutron, the integral of speed times the number of neutrons within the region (i.e. one or zero) over the time interval equals the sum of one times the track length within the region plus zero times the track length outside the region. Summing over all neutrons shows that the expected mean track length equals the flux integrated over the region and the time interval.

In accordance with these conclusions, the equations of Chaptis III will be shown here so imply that the $M_{1} ' s$, but not the $M_{2}{ }^{\prime} s$, of the two estimators are equal. It is sufficien: to prove the $Q_{1}$ 's, but not the $Q_{2}^{\prime} s$, are equal, for $T\left(P, P^{+}\right), \sigma\left(P^{+}, P^{\prime}\right), B\left(P^{\prime}, P^{\prime \prime}\right), E\left(P^{\prime}, P^{\prime \prime}\right)$, and $\varepsilon_{k}\left(P^{\prime}, P_{1}, \cdots, P_{k}\right)$ in eq. (108) do not depend on the scoring distributions 
$P\left(P, P^{\prime}, s\right)$. An analog game will be considered here but one can use the results of eq. (142) to generailize to a non-analog game.

Let $x^{\prime}$ be the coordinate along $\vec{\Omega}$, the direction of the neutron's flight. Let

$\vec{r}^{\prime}-\vec{r}=\left(x^{\prime}, y^{\prime}, z^{\prime}\right)$

so that the neutron's position is described in a Cartesian coordinate system where the $X^{\prime}$ axis lies along $\vec{\Omega}$. The transmission probability that a particle travels a distance $x^{\prime}$ through a medium (of macroscopic total cross section $\sigma$ ) is

$T\left(R, x^{\prime}\right)=T\left(\vec{r}, \vec{r}^{\prime}, \vec{v}\right)= \begin{cases}\exp \left[-\int_{0}^{x^{\prime}} \sigma(\vec{r}+\vec{r} h, \vec{v}) d h\right] & \text { if } x^{\prime} \geq 0 \\ 0 & \text { if } x^{\prime}<0\end{cases}$

That is

$T\left(R, R^{\prime}\right)= \begin{cases}\delta\left(\vec{v}-\vec{v}^{\prime}\right) \delta\left(y^{\prime}\right) \delta\left(z^{\prime}\right) T\left(R, x^{\prime}\right) & \text { if } x^{\prime} \geq 0 \\ 0 & \text { if } x^{\prime}<0\end{cases}$

Differentiating eq. (146)

$$
\frac{d T\left(R, x^{\prime}\right)}{d x^{\prime}}=\left\{\begin{array}{cl}
-\sigma\left(\vec{r}+\vec{\Omega} x^{\prime}, \vec{v}\right) T\left(R, x^{\prime}\right) & \text { if } x^{\prime} \geq 0 \\
0 & \text { if } x^{\prime}<0
\end{array}\right.
$$

Now consider a spatially convex region $\Gamma$ of $(\vec{r}, \vec{v})$ space (it will be shown at the end of this section that the results are valid even if $\Gamma$ is not spatialiy convex). Let the possible path length inside $\Gamma$ extend from $x_{3}$ to $x_{2}$ and let $\Gamma$ extend over all velocities. 


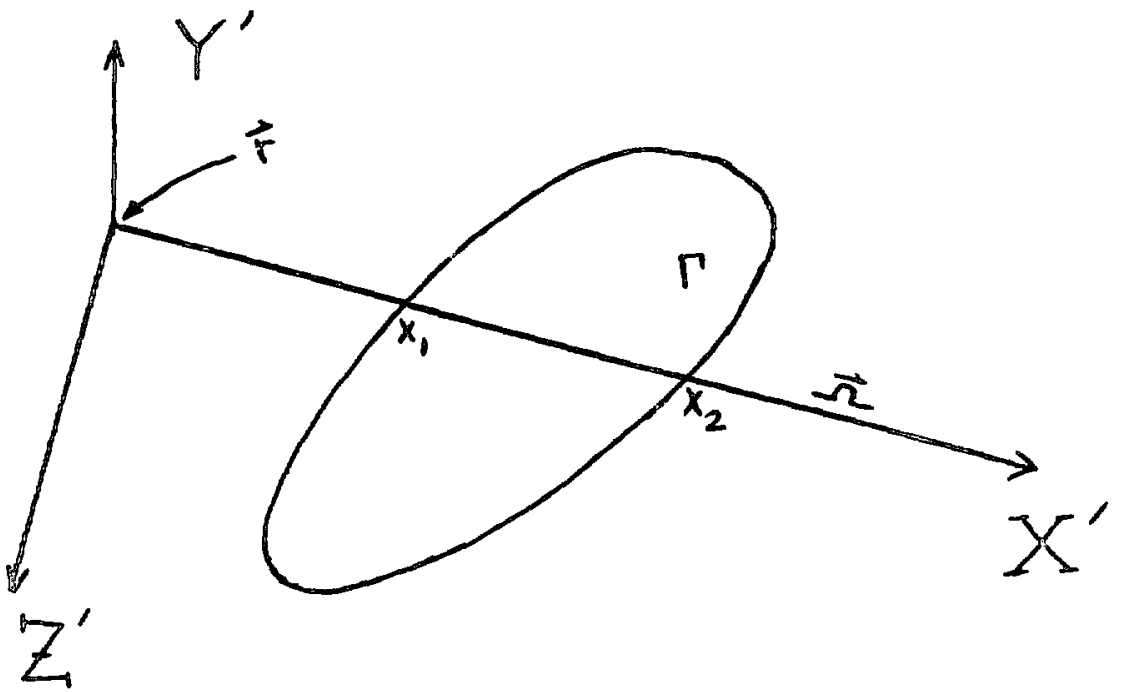

If $v t$ is less than the distance to $\Gamma_{0}$ then the neutron cannot reach $r$ in time $t$ and $x_{j}=x_{2}=0$. If $\vec{r}$ is inside $r$ then $x_{p}=0$, and if $\vec{r}+\vec{v} t$ is inside $r$ then $x_{2}=v t$. If the neutron's flight path does not intersect $\Gamma$ then $x_{1}=x_{2}=0$ and no score is possible.

Q's for Collision Estimator

For the collision estimator each collision inside $r$ contributes a score $1 / \sigma$ and a free flight for the entire time remaining conitributes zero. Thus

$P_{k}\left(P_{0}, P^{0}, s\right)=\left\{\begin{array}{ll}\delta(s-1 / \sigma) & R^{\prime} \text { in } \Gamma \\ \delta(s) & R^{\prime} \text { not in } \Gamma\end{array}\right\} k=0,8,2 \ldots \ldots$

$P_{d}\left(P_{D} P^{\prime}, s\right)=\delta(s)$ 
Let

$\sigma\left(x^{\prime}, \vec{v}\right)=\sigma\left(\vec{r}+\vec{\Omega} x^{\prime}, \vec{v}\right)$

Using equations (146)-(151) with ald weights unity, eqs. (127) and

(130) become

$Q_{1}(R, Y, t)=\int_{x_{1}}^{x_{2}} d x^{\prime} T\left(R, x^{\prime}\right)$

$Q_{2}(R, J, t)=\int_{x_{1}}^{x_{2}} d x^{\prime} T\left(R, x^{\prime}\right)\left[\frac{1}{\sigma\left(x^{\prime}, \bar{v}\right)}+2 \int d R^{\prime \prime} E\left(R^{\prime}, R^{\prime \prime}\right) M_{1}\left(R^{\prime \prime}, 1, t-t^{\prime}\right)\right.$

$+\sum_{k=2}^{\infty} \int d R_{1} \cdots \iint R_{k} \varepsilon_{k}\left(R^{0}, R_{1}, \cdots, R_{k}\right) \mid 2 \sum_{\ell=1}^{k} M_{1}\left(R_{\ell}, 1, t-t^{\prime}\right)+2 \sigma\left(x^{0}, \vec{v}\right)$

$\left.\left.\sum_{\ell=2}^{k} M_{j}\left(R_{\ell}, 1, t-t^{0}\right) \cdot \sum_{j=1}^{\ell-\pi} M_{j}\left(R_{j}, 1, t-t^{\prime}\right)\right\}\right]$

\section{Q's for Track Length Estimator}

For the track length estimator each track the neutron leaves in

$\Gamma$ contributes a score equal to the length. of the track. Thus

$P_{k}\left(P, P^{0}, s\right)=P_{d}\left(P, P^{\prime}, s\right)=p\left(P, P^{\prime}, s\right)$ for $k=0,1,2 \cdots$, where

$P\left(P, P^{\prime}, s\right)= \begin{cases}\delta(s) & \text { if } x^{\prime}<x_{1} \\ \delta\left(s-\left(x^{0}-x_{1}\right)\right) & \text { if } x_{1} \leq x^{\prime} \leq x_{2} \\ \delta\left(s-\left(x_{2}-x_{1}\right)\right) & \text { if } x_{2}<x^{\prime}\end{cases}$ 
Using eqs. (146), (147), (148), (154), and (155) with all weights unity eq. (127) becomes

$$
\begin{aligned}
& q_{1}(R, 1, t)=\left(x_{2}-x_{1}\right) T(R, v t)+\int_{x_{1}}^{x_{2}} d x^{\prime} T\left(R, x^{\prime}\right) \sigma\left(x^{\prime}, \vec{v}\right)\left(x^{\prime} \cdots x_{1}\right) \\
& +\int_{x_{2}}^{v t} d x^{\prime} T\left(R, x^{\prime}\right) \sigma\left(x^{\prime}, \vec{v}\right)\left(x_{2}-x_{1}\right) .
\end{aligned}
$$

Substituting eq. (148) into eq. (156) yields

$$
\begin{aligned}
& Q_{1}(R, T, t)=\left(x_{2}-x_{1}\right) T(R, v t)+\int_{x_{1}}^{x_{2}}\left(x^{\prime}-x_{1}\right)\left(\frac{-d T\left(R, x^{\prime}\right)}{d x^{\prime}}\right) d x^{\prime} \\
& -\left(x_{2}-x_{1}\right) \int_{T\left(R, x_{2}\right)}^{T(R, v t)} d T .
\end{aligned}
$$

Integrating the first integral by parts,

$$
Q_{1}(R, 1, t)=\int_{x_{1}}^{x_{2}} T\left(R, x^{\prime}\right) d x^{\prime}
$$

As expected, the $Q_{1}$ for the track length estirnator equals the $Q_{1}$ for the collision estimator. Substituting eqs. (146) and (147) into eq. (130), noting that $w_{c 0}$ is zero for an analog game, and using eqs. (82) (83) to collect terms in $s^{2}$ : 


$$
\begin{aligned}
& Q_{2}(R, 1, t)=T(R, v t)\left(x_{2}-x_{1}\right)^{2}+\int_{x_{1}}^{x_{2}} d x^{\prime} T\left(R, x^{\prime}\right) Q\left(x^{\prime}, \vec{v}\right)\left(x^{\prime}-x_{1}\right)^{2} \\
& +\int_{x_{2}}^{v t} d x^{\prime} T\left(R, x^{\prime}\right) \sigma\left(x^{\prime}, \vec{v}\right)\left(x_{2}-x_{1}\right)^{2}+\int_{x_{1}}^{x_{2}} d x^{\prime} T\left(R, x^{\prime}\right) \sigma\left(x^{\prime}, \vec{v}\right) \\
& x\left[2\left(x^{\prime}-x_{1}\right) \int d R^{\prime \prime} E\left(R^{\prime}, R^{\prime \prime}\right) M_{j}\left(R^{\prime \prime}, 1, t-t^{\prime}\right)+\int d R_{1} \cdots \int d R_{k} \varepsilon_{k}\left(R^{\prime}, R_{1}, \cdots, R_{k}\right)\right. \\
& x\left\{2\left(x^{\prime}-x_{1}\right) \sum_{\ell=1}^{k} M_{1}\left(R_{\ell}, l, t-t^{\prime}\right)+2 \sum_{\ell=2}^{k} M_{1}\left(R_{\ell}, 1, t-t^{\prime}\right)\right. \\
& \left.x \sum_{j=1}^{l-1} M_{1}\left(R_{j}, l, t-t^{\prime}\right) \mid\right]+\int_{x_{2}}^{v t} d x^{\prime} T\left(R, x^{\prime}\right) \sigma\left(x^{\prime}, \vec{v}\right)\left[2\left(x_{2}-x_{1}\right)\right. \\
& x \int d R^{\prime \prime} E\left(R^{\prime}, R^{\prime \prime}\right) M_{1}\left(R^{\prime \prime}, 1, t-t^{\prime}\right)+\int d R_{1} \cdots \int d R_{k} E_{k}\left(R^{\prime}, R_{1}, \cdots, R_{k}\right) \\
& x\left\{2\left(x_{2}-x_{1}\right) \sum_{\ell=1}^{k} M_{1}\left(R_{\ell}, 1, t-t^{\prime}\right)+2 \sum_{\ell=2}^{k} M_{1}\left(R_{\ell}, 1, t-t^{\prime}\right)\right. \\
& \left.\left.x \sum_{j=1}^{\ell-1} M_{1}\left(R_{j}, 1, t-t^{0}\right)\right\}\right]
\end{aligned}
$$

$Q_{2}$ for the collision estimator and $Q_{2}$ for the track length estimator are not generally equal, as can be seen by comparing the case in which $\sigma$ approaches zero in the medium

$Q_{2}+\left(x_{2}-x_{1}\right)^{2}$ for the track length estimator

(160)

$Q_{2}+\infty \quad$ for the collision estimator

(161)

64 
Thus for optically thin systems the $M_{2}$ for the track length estimator will be much less than the $M_{2}$ for the collision estimator, resulting in the track length estimator having a much smaller variance than the collision estimator.

The above analysis can be applied to non-convex regions as well as to convex regions. Any bounded region which arises in practice can be approximated, to any accuracy desired, by a finite number of convex parts (see Appendix D). For either estimator the $Q_{r}$ for any region equals the sum of the $Q_{r}$ 's for its parts.

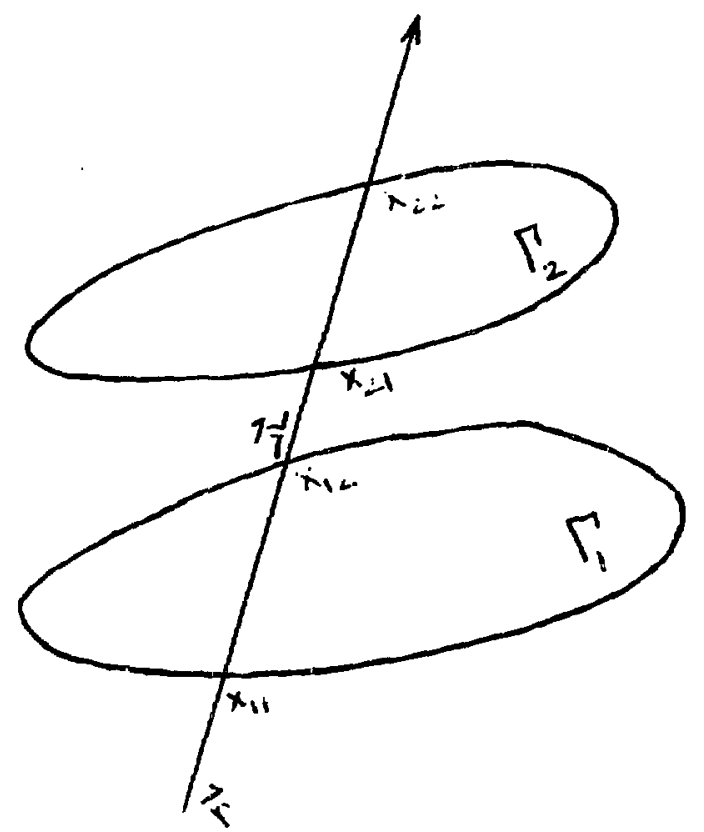

Since the equation for $M_{r}$ is linear, the $M_{r}$ for any region equals the sum of the $M_{r}$ 's for its parts. (The $M_{1}$ fed into the equation for $Q_{2}$ in any subregion should be the $M_{7}$ for the entire region $\Gamma$ and not the $M_{1}$ for the subregion.) 
B. One-Speed Infinite Medium Equations

Consider a one speed problem in an infinite homogeneous medium (with macroscopic total cross section $\sigma^{*}$ ) where an event score depends only on the relative coordinate, $\vec{r}^{\prime}-\vec{r}$, between the next event point and the departure point. Let

$A_{k}^{*}\left(R^{\prime}\right)=A_{k}^{*}(k=0,1,2, \cdots)$ independent of $R^{\prime}$

So that all cross sections are constant.

Suppose one attempts to solve this problem using a non-analog Monte Carlo Method in which

$\sigma\left(R^{\prime} !=\sigma\right.$ independent of $R^{\prime}$

$A_{k}\left(R^{\prime}\right)=A_{k}(k=0,1,2, \cdots)$ independent of $R^{\prime}$

With these choices, the fictitious Monte Carlo medium looks infinite and homogeneous just as the real medium does. Since the Monte Carlo medium is infinite and homogeneous $M_{r}(\vec{r}, \vec{\Omega}, w, t)$ should be independent of $\vec{r}$ and $\vec{\Omega}$. That is, any location looks the same as any other location since there are no reference points, or

$M_{r}(\vec{r}, \vec{\Omega}, w, t)=M_{r}(w, t)$

+ for simplicity weight cutoff is not considered (i.e. $w_{c o}=0$ ). 
The cross sections are all constant so the probability of an eventless transmission from $\vec{r}$ to $\vec{r}^{\prime}$ is $e^{-\sigma\left|\vec{r}^{\prime}-\vec{r}\right|} \mid \begin{aligned} & \text { if } \vec{r}^{\prime}-\vec{r} \text { is along the neutron's flight path } \\ & \text { otherwise }\end{aligned}$

Let

$\vec{r}^{\prime}-\vec{r}^{\prime}=\left(x^{\prime}, y^{\prime}, z^{\prime}\right)$

If $x^{\prime}$ is taken along $\vec{\Omega}$, the direction of the neutron's flight, then the transmission probability kernel becomes:

$T\left(P, P^{\prime}\right)=e^{-\sigma x^{\prime}} \delta\left(\vec{\Omega}-\vec{\Omega}^{\prime}\right) \delta\left(y^{\prime}\right) \delta\left(z^{\prime}\right) \delta\left(w^{\prime}-w_{t} w\right)$

where

$\sigma\left(R^{\prime}\right)=\sigma$

The free flight karnel becornes

$D\left(P, P^{\prime}, t\right)=e^{-\sigma x^{\prime}} \delta\left(\vec{\Omega}-\vec{\Omega}^{\prime}\right) \delta\left(y^{\prime}\right) \delta\left(z^{\prime}\right) \delta\left(w^{\prime}-w^{\prime} w\right) \delta\left(x^{\prime}-v t\right)$

Substituting eqs. (82), (84), (86), (168), (169), and (170) into

eqs. (125), (127), and (130) results in eqs. (171), (172), and (173).

$M_{r}(w, t)=\sum_{k=1}^{\infty} \int_{0}^{v t} d x^{\prime} \sigma e^{-\sigma x^{\prime}} k A_{k} M_{r}\left(w w w_{t} w^{w} \varepsilon_{k}, t-t^{\prime}\right)+Q_{r}(w, t)$ 


$$
\begin{aligned}
& Q_{1}=\int d x^{\prime} e^{-\sigma x^{\prime}} \delta\left(x^{\prime}-v t\right) \int d s p_{d}\left(x^{\prime}, w w w_{t}, s\right) s \\
& +\sum_{k=0}^{\infty} \int_{0}^{v t} d x^{\prime} \sigma e^{-\sigma x^{\prime}} \int d s p_{k}\left(x^{\prime}, w w_{t} w_{\sigma}, s\right) A_{k} s \\
& Q_{2}=\int d x^{\prime} \delta\left(x^{\prime}-v t\right) \int d s p_{d}\left(x^{\prime}, w w_{t}, s\right) e^{-\sigma x^{\prime}} s^{2} \\
& +\sum_{k=0}^{\infty} \int_{0}^{v t} d x^{\prime} \sigma e^{-\sigma x^{\prime}} \int d s p_{k}\left(x^{\prime}, w w_{t} w_{\sigma}, s\right) A_{k} \\
& x\left\{s^{2}+2 s k M_{1}\left(w w_{t}{ }^{W} \sigma^{W} \varepsilon_{k}, t-t^{\prime}\right)+k(k-1) M_{1}^{2}\left(w w_{t}{ }^{W} \sigma^{W} \varepsilon_{k}, t-t^{\prime}\right)\right\}
\end{aligned}
$$

Let

$$
\begin{aligned}
& y=\sigma v t \\
& y^{\prime}=\sigma v t^{\circ}=\sigma x^{\circ}
\end{aligned}
$$

Changing variables $t$ and $t^{\prime}$ to $y$ and $y^{\prime}$ results in

$$
\begin{aligned}
& M_{r}(w, y)=\sum_{k=1}^{\infty} k A_{k} \int_{0}^{y} d y^{\prime} e^{-y^{\prime}} M_{r}\left(W w_{t} w \sigma^{w} \varepsilon_{k}, y-y^{\prime}\right)+Q_{r} \\
& Q_{1}=e^{-y} \int d s p_{d}\left(y, w w_{t}, s\right) s \\
& +\sum_{k=0^{\prime}}^{\infty} \int_{0}^{y} d y^{\prime} e^{-y^{\prime}} \int d s p_{k}\left(y^{\prime}, w w_{t} w_{\sigma^{\prime}}, s\right) A_{k} s \\
& Q_{2}=e^{-y} \int d s p_{d}(y, m w t, s) s^{2} \\
& +\sum_{k=0}^{\infty} \int_{0}^{y} d y^{\prime} e^{-y^{\prime}} \int d s p_{k}\left(y^{\prime}, w w_{t} w_{\sigma}, s\right) A_{k}\left\{s^{2}\right. \\
& \left.+2 s k M_{1}\left(w w_{t} w_{\sigma} w_{\varepsilon_{k}}, y-y^{\prime}\right)+k(k-1) M_{1}^{2}\left(m w_{t} w_{\sigma} w_{k}, y-y^{\prime}\right)\right\}
\end{aligned}
$$

68 
From eqs. (176) and (A6)

$$
M_{r}(w, y)=\sum_{k=1}^{\infty} k A_{k} T\left[M_{r}\left(w w_{t} w_{0} w_{E_{k}}, y\right)\right]+Q_{r}
$$

Expanding $M_{r}$ and $Q_{r}$ in power series

$$
\begin{aligned}
& M_{r}(w, y)=w^{r} \sum_{n=0}^{\infty} a_{n} y^{n} \\
& Q_{r}(w, y)=w^{r} \sum_{n=0}^{\infty} b_{n} y^{n}
\end{aligned}
$$

Substituting eqs. (180) and (180A) into eq. (179):

$$
w^{r} \sum_{n=0}^{\infty} a_{n} y^{n}=w^{r} \sum_{k=1}^{\infty} k A_{k}\left(w_{t} w_{\sigma} w_{\varepsilon_{k}}\right)^{r} T\left[\sum_{n=0}^{\infty} a_{n} y^{n}\right]+w^{r} \sum_{n=0}^{\infty} b_{n} y^{n}
$$

Let

$$
\alpha=\sum_{k=1}^{\infty} k A_{k}\left(w_{t} w_{\sigma} w_{k}\right)^{r}
$$

then using eq. (A10)

$$
\sum_{n=0}^{\infty} a_{n} y^{n}=\alpha \sum_{n=0}^{\infty}(-1)^{n+1} n ! a_{n} \sum_{j=n+1}^{\infty}(-1)^{j} \frac{y^{j}}{j !}+\sum_{n=0}^{\infty} b_{n} y^{n}
$$

Collecting like powers

$$
a_{n}=\frac{(-1)^{n+1} \alpha}{n !} \sum_{m=1}^{n-1}(-1)^{m} m \cdot a_{m}+b_{n}
$$

Hence as long as $Q_{r}$ can be expanded in a power series the solution to eq. (125) is given ty eq. (184). Thus solving for the expected errors, in a problem where the moments are dependent only on time and weight, has been reduced to solving for the $a_{n}$, given the $b_{n}$.

69 
This porser series method fails, of course, when $Q_{r}$ is not anaiytic in $y . Q_{r}$ need not be analytic. Suppose

$P_{k}\left(y^{\prime}, w_{t} w_{\sigma}, s\right)=\delta(s)$ and

$P_{d}\left(y, w w_{t}, s\right)=\delta\left(s-w w_{t} H\left(y-y_{0}\right)\right)$.

then eq. (177) becomes

$Q_{1}=w_{t} H\left(y-y_{0}\right) e^{-y}$

Thus $Q_{1}$ is independent of space (recall $y$ is a relative coordinate) and angle, but not analytic in $y$. 
C. Theoretical Comparison of Four Estimators for Collisions

The variances of four Nonte Carlo techniques for obtaining the mean number of collisions a particle (and its secondaries) makes in time $t$ are compared below using the equations of section $B$.

Consider an infinite homogeneous medium with the following properties:

$A_{0}^{*}=1 / 3 ;$ a third of the collisions result in absorption

$A_{1}^{*}=1 / 3$; a third of the collisions result in scattering

$A_{2}^{*}=1 / 3 ;=$ third of the collisions result in two exiting particles

$A_{m}^{\star}=0$ for $m \geq 3$; no collision results in more than two exiting particles

$\sigma^{*}=$ macroscopic cross section

$\alpha^{\star}=\sum_{k=1}^{\infty} k A_{k}^{*}=1$

The following four estimators for collisions are compared:

1) analog collision estimator

2) track length estimator

3) survival biased collision estimator

4) survival biased track length estimator

A.1 kernels, score distributions, and weight multipliers are chosen in acsordance with eqs. (133), (136), and (137) so that eq. (142) holds when biased kerneis are used, thus ensuring estimators 1) and 3) have the same $M_{1}$ and estimators 2) and 4) have the same $M_{1}$. Estimators 1) 
and 2) are the same as the estimators described in IY-A except that the scores in 11 and 2) are a constant $\sigma$ times the scores in IV-A. Thus the $M_{1}$ 's for the estimators 1) and 2) will be $\sigma$ times the $M_{1}$ 's for the corresponding estimators in IV-A. The $M_{1}$ 's in IV-A are equal; hence the $M_{1}$ 's for estimators 1) and 2) are equal. Thus estimators 1), 2), 3) and 4) can be expected to have the same means but different variances. One possible point of confusion should be addressed before obtaining the variances for the four estimators. Quantities such as $T^{\star}, \sigma^{*}$, $A^{\star}, E^{\star}$, and $\varepsilon_{k}^{*}$ which describe the medium must be independent of biasing schemes and choice of estimator. However, the score distributions $P^{\star}\left(P_{,} P^{\prime}, s\right)$ (used with unbiased kernels) do depend on the choice of estimator. Thus the $p^{*}\left(P, P^{\prime}, s\right)$ for estimators 1$)$ and 3 ) are different from the $P^{*}\left(P, P^{\prime}, S\right)$ for estimators 2$)$ and 4$)$. 


\section{Game 1 Analog Collision Estimator}

Unbiased kernels are used and the particle's weight is scored at every collision. For an analog game a particle's weight never changes.

$$
\begin{aligned}
& A_{0}\left(R^{\prime}\right)=A_{0}^{\star}\left(R^{\prime}\right) \quad A_{0}=A_{0}^{\star}=1 / 3 \\
& T\left(R, R^{+}\right)=T^{*}\left(R, R^{+}\right) \quad W_{0}=1 \\
& \sigma\left(R^{\prime}\right)=\sigma^{*}\left(R^{\prime}\right) \quad W_{\sigma}=1
\end{aligned}
$$

$$
E\left(R^{\prime}, R^{\prime \prime}\right)=E^{*}\left(R^{\prime}, R^{\prime \prime}\right) \quad W_{E_{1}}=1 \quad A_{1}=A_{1}^{*}=1 / 3
$$

$\varepsilon_{2}\left(R^{\prime}{ }_{0} R_{1}, R_{2}\right)=\varepsilon_{2}^{\star}\left(R^{\prime}, R_{1}, R_{2}\right) \quad W_{\varepsilon_{2}}=1 \quad A_{2}=A_{2}^{*}=1 / 3$

$D\left(R, R^{+}, t\right)=D^{\star}\left(R, R^{+}, t\right)$

$P_{k}\left(P, P^{\prime}, s\right)=P_{k}^{*}\left(P^{\prime}, P^{\prime}, s\right)=\delta\left(s-w^{\prime}\right) \quad k=0,1,2$

$p_{d}\left(P, P^{\prime}, s\right)=P_{d}^{*}\left(P, P^{\prime}, s\right)=\delta(s)$

Substituting relations (185)-(197) into eq. (177)

$Q_{1}=\int_{0}^{y} e^{-y^{\prime}} w\left\{A_{0}+A_{1}+A_{2}\right\} d y^{\prime}=w \int_{0}^{y} e^{-y^{\prime}} d y^{\prime}$

From eq. (182) and (191)-(194)

$\alpha=\sum_{k=1}^{\infty} k A_{k} w_{t} w_{\sigma}{ }^{w} E_{k}=1$

Using eqs. (A6) and (A10) eq. (198) bacomes

$Q_{1}=w T[1]=w \sum_{j=1}^{\infty}(-1)^{j+1} \frac{y^{j}}{j !}$ 
therefore from eq. (180A)

$$
b_{n}=\frac{(-1)^{n+1}}{n !} \quad n \geq 1
$$

From eqs. (184) and (201)

$$
a_{n}=\frac{(-1)^{n+1}}{n !}\left(\sum_{m=1}^{n-1}(-1)^{m} m: a_{m}+1\right)
$$

Solving eq. (202) for the first few $a_{n}$ :

$$
\begin{aligned}
& a_{1}=1 \\
& a_{2}=0 \\
& a_{3}=0
\end{aligned}
$$

Inductive Proof that $a_{n}=0$ for $n \geq 2$

Suppose $a_{n}=0$ then from eq. (202)

$$
\sum_{m=1}^{n-1}(-i)^{m} m ! a_{m}+1=0
$$

From eq. (202)

$$
\begin{aligned}
& a_{n+1}=\frac{(-1)^{n+2}}{(n+1) !}\left((-1)^{n} n ! a_{n}+\sum_{m=1}^{n-1}(-1)^{m} m a_{m}+1\right) \\
& a_{n+1}=\frac{n !}{(n+1) !} a_{n}=0
\end{aligned}
$$

Hence $a_{n}=0$ implies $a_{n+1}=0$; but $a_{2}=0$ hence $a_{n}=0$ for $n \geq 2$.

Thus by eq. ( 180$)$

$M_{1}(w, y)=w y=w o v t$

Using eqs. (190)-(197), (203), and (A6), eq. (178) becomes

$Q_{2}=\left[\left(A_{0}+A_{1}+A_{2}\right) T[l]+\left(2 A_{1}+4 A_{2}\right) T[y]+2 A_{2} T\left[y^{2}\right]\right] w^{2}$

(204)

74 
Using eq. (A10)

$$
\begin{aligned}
& w^{-2} q_{2}=\sum_{n=1}^{\infty}(-1)^{n+1} \frac{y^{n}}{n !}+2 \sum_{n=2}^{\infty}(-1)^{n} \frac{y^{n}}{n !}+\frac{2}{3} \cdot 2 \sum_{n=3}^{\infty}(-1)^{n+1} \frac{y^{n}}{n !} \\
& w^{-2} 0_{2}=y+\frac{1}{2} y^{2}+\frac{1}{3} \sum_{n=3}^{\infty}(-1)^{n+1} \frac{y^{n}}{n !}
\end{aligned}
$$

By eq. (180A)

$$
\begin{aligned}
& b_{1}=1 \\
& b_{2}=1 / 2 \\
& b_{n}=\frac{1}{3} \frac{(-1)^{n+1}}{n !} \text { for } n \geq 3
\end{aligned}
$$

From eqs. (184), (199), and (207)

$$
\begin{aligned}
& a_{1}=1 \\
& a_{2}=1 \\
& a_{n}=\frac{(-1)^{n+1}}{n !}\left[\sum_{m=1}^{n-1}(-1)^{m} m: a_{m}+\frac{1}{3}\right] n \geq 3
\end{aligned}
$$

Using eq. (208)

$$
\begin{aligned}
& a_{3}=\frac{1}{3 !} \frac{4}{3} \\
& a_{4}=0
\end{aligned}
$$

Proof (by induction) that $a_{n}=0$ for $n \geq 4$

Suppose $a_{n}=0$; then from eq. (208)

$\sum_{m=1}^{n=1}(-1)^{m} m: a_{m}+\frac{1}{3}=0$

Hriting eq. (208) for $n+1$

$a_{n+1}=\frac{(-1)^{n}}{(n+1) !}\left((-1)^{n} n ! a_{n}+\sum_{m=1}^{n-1}(-1)^{i n} m ! a_{m}+\frac{1}{3}\right)$ 
Using $a_{n}=0$ and eq. (208A) in eq. (208B) results in $a_{n+1}=0$. Thus $a_{4}=0$ implies $a_{n}=0$ for $n \geq 4$.

From eq. (180)

$M_{2}(w, y)=w^{2}\left(y+y^{2}+\frac{2}{9} y^{3}\right)$

Using eqs. (203) and (208C)

VAR $=M_{2}(w, y)-M_{1}^{2}(w, y)=w^{2}\left(y+\frac{2}{9} y^{3}\right)$

\section{Game 2 Track Length Estimator}

Unbiased kernels are used and the event score generated by a particle of unit weight is $\sigma$ times the distance travelled between events.

$$
\begin{array}{ll}
A_{0}\left(R^{\prime}\right)=A_{0}^{*}\left(R^{i}\right) & A_{0}=A_{0}^{*} \\
T\left(R, R^{+}\right)=T^{*}\left(R, R^{+}\right) & W_{t}=1 \\
\sigma\left(R^{\prime}\right)=\sigma^{*}\left(R^{0}\right) & W_{\sigma}=1
\end{array}
$$

$E\left(R^{\prime}, R^{\prime \prime}\right)=E^{\star}\left(R^{\prime}, R^{\prime \prime}\right) \quad W_{\varepsilon_{1}}=1 \quad A_{1}=A_{1}^{*}$

$\varepsilon_{2}\left(R^{\prime}, R_{1}, R_{2}\right)=\varepsilon_{2}^{\star}\left(R^{\prime}, R_{1}, R_{2}\right) \quad w_{\varepsilon_{2}}=1 \quad A_{2}=A_{2}^{\star}$

$D\left(R, R^{+}, t\right)=D^{*}\left(R, R^{+}, t\right)$

$P_{k}\left(P, P^{\prime}, s\right)=P_{k}^{\star}\left(P, P^{\prime}, s\right)=\delta\left(s-w^{\prime} E x^{\prime}\right)=\delta\left(s-w^{\prime} y^{\prime}\right) \quad k=0,7,2$

$P_{d}\left(P, P^{\prime}, s\right)=P_{d}^{*}\left(P_{,}, P^{\prime}, s\right)=\delta\left(s-w^{\prime} \sigma x^{\prime}\right)=\delta\left(s-w^{\prime} y^{\prime}\right)$

From eqs. (177) and (216)

$q_{1}=w\left[y e^{-y}+\int_{0}^{y} d y^{\prime} e^{-y^{\prime}}\left\{A_{0} y^{\prime}+A_{1} y^{\prime}+A_{2} y^{\prime}\right\}\right]$ 
Using eq. (A7) and (AB)

$$
\begin{aligned}
& Q_{1} / w=y e^{-y}+v[y]=y e^{-y}+e^{-y} \sum_{j=2}^{\infty} \frac{y^{j}}{j !} \\
& Q_{1} / w=e^{-y} \sum_{j=1}^{\infty} \frac{y^{j}}{j !}=e^{-y}\left(e^{y}-1\right)=\sum_{j=1}^{\infty}(-1)^{j+1} \frac{y^{j}}{j !}
\end{aligned}
$$

and from eq. (182) with $r=1$

$$
\alpha=\sum_{k=1}^{\infty} k A_{k}{ }^{W} \varepsilon_{k}{ }^{W}{ }^{W} t=1
$$

$a_{1}$ (and hence $b_{n}$ ) and $\alpha$ are the same as in game 1 . Thus following the same steps as in game 1 yields

$$
M_{1}(w, y)=w y
$$

From eqs. (17\%) and (221)

$$
\begin{aligned}
Q_{2} & =w^{2} y^{2} e^{-y}+w^{2} \int_{0}^{y} d y^{\prime} e^{-y}\left\{A_{0} y^{\prime 2}+A_{1} y^{\prime 2}+A_{2} y^{\prime 2}\right. \\
& \left.+2 A_{1} y^{\prime}\left(y-y^{\prime}\right)+4 A_{2} y^{\prime}\left(y-y^{\prime}\right)+2 A_{2}\left(y-y^{\prime}\right)^{2}\right\}
\end{aligned}
$$

Using eqs. (A6) - (ATO)

$$
\begin{aligned}
& w^{-2} Q_{2}=y^{2} E^{-y}-V\left[y^{2}\right]+2 y V[y]+\frac{2}{3} T\left[y^{2}\right] \\
& w^{-2} Q_{2}=\frac{2}{3}\left(e^{-y}-1\right)+\frac{2}{3} y^{2}+\frac{2}{3} y \\
& w^{-2} Q_{2}=y^{2}+\frac{2}{3} \sum_{n=3}^{\infty}(-1)^{n} \frac{y^{n}}{n !} \\
& b_{1}=0 \quad b_{2}=1 \text { and for } n \geq 3 \quad b_{n}=\frac{2}{3}(-1)^{n} \frac{1}{n !}
\end{aligned}
$$


From eqs. (182) with $r=2$

$\alpha=\sum_{k=1}^{\infty} k A_{k} w_{k}^{2} \varepsilon_{k} w_{t}^{2} w_{\sigma}^{2}=1$

Using eq. (184) $a_{1}=0, a_{2}=1$, and for $n \geq 3$

$a_{n}=\frac{(-1)^{n+1}}{n !}\left[\sum_{m=1}^{n-1}(-1)^{m_{m} !} a_{m}-\frac{2}{3}\right]$

Thus $a_{3}=\frac{1}{3 !} \frac{4}{3}, a_{4}=0, a_{5}=0$, and $a_{6}=0$.

Proof (by induction) that $a_{n}=0$ for $n \geq 4$

Suppose $a_{n}=0 ;$ then from eq. (228)

$\sum_{m=1}^{n-1}(-1)^{m} m ! a_{m}-\frac{2}{3}=0$

Again from eq. (228)

$a_{n+1}=\frac{(-1)^{n+2}}{(n+1) !}\left[(-1)^{n} n ! a_{n}+\sum_{m=1}^{n-1}(-1)^{m} m ! a_{m}-\frac{2}{3}\right]$

Thus using eq. (229) in eq. (230) it follows that $a_{n}=0$ implies $a_{n+1}=0$. But $a_{4}=0$; hence for $n \geq 4, a_{n}=0$.

Thus from eq. (i80)

$M_{2}(w, y)=w^{2}\left(y^{2}+\frac{2}{9} y^{3}\right)$, and

VAR $=M_{2}(w, y)-M_{7}^{2}(w, y)=w^{2} \frac{2}{9} y^{3}$ 


\section{Game 3 Survival Biased Collision Estimator}

The exit kernels are biased so that absorption cannot occur. This is known as absorption by weight reduction, or survival biasing. A particle of weight $w$ generates a score of $w$ every time the particle collides.

$A_{0}\left(R^{\prime}\right)=0$ no absorption, called "survival biased"

$$
\begin{array}{ll}
T\left(R, R^{+}\right)=T^{*}\left(R, R^{+}\right) & w_{t}=1 \\
\sigma\left(R^{\prime}\right)=\sigma^{*}\left(R^{\prime}\right) & w_{\sigma}=1
\end{array} \mid \begin{aligned}
& \text { next collision location } \\
& \text { ront biased }
\end{aligned}
$$

$E\left(R^{\prime}, R^{\prime \prime}\right)=\frac{3}{2} E^{\star}\left(R^{\prime}, R^{\prime \prime}\right) \quad W_{\varepsilon_{g}}=\frac{2}{3} \quad A_{1}=\frac{3}{2} A^{\star}{ }_{1}=\frac{1}{2}$

$\varepsilon_{2}\left(R^{\prime}, R_{1}, R_{2}\right)=\frac{3}{2} \varepsilon_{2}^{\star}\left(R^{\prime}, R_{1}, R_{2}\right) \quad w_{\varepsilon_{2}}=\frac{2}{3} \quad A_{2}=\frac{3}{2} A_{2}^{*}=\frac{1}{2}$

$P_{k}\left(P, P^{\prime}, s\right)=P_{k}^{*}\left(P, P^{\prime}, s\right)=\delta\left(s-w^{\prime}\right) \quad k=0,1,2$

$P_{d}\left(P, P^{\prime}, s\right)=P_{d}^{*}\left(P, P^{\prime}, s\right)=\delta(s)$

Substituting eqs. (232) - (237A) into eq. (177) yieids

$Q_{1}=w \int_{0}^{y} d y^{\prime} e^{-y^{\prime}}$

Using eqs. (A6) and (A10)

$Q_{1} / w=T[1]=\sum_{n=1}^{\infty}(-1)^{n+1} \frac{y^{n}}{n !}$

Substituting eqs. (233) - (236) into eq. (182) with $r=1$ yields

$a=\frac{1}{2} \cdot \frac{2}{3}+2 \cdot \frac{1}{2} \cdot \frac{2}{3}=1$ 
$Q_{1}$ and $a$ are the same as they were in game 1; thus following the same steps as in game 1 yields

$M_{1}(w, y)=w y$

Substituting eqs. (232) - (237A) and (241) into eq. (178) yields

$Q_{2}=w^{2} \int_{0}^{y} d y^{\prime} e^{-y^{\prime}}\left[\frac{1}{2}\left(1+\frac{4}{3}\left(y-y^{\prime}\right)+1+\frac{8}{3}\left(y-y^{\prime}\right)+\frac{8}{9}\left(y-y^{\prime}\right)^{2}\right)\right]$

Using eqs. (AG) and (A10)

$w^{-2} Q_{2}=T[1]+2 T[y]+\frac{4}{9} T\left[y^{2}\right]$

$w^{-2} Q_{2}=y+\frac{1}{2} y^{2}+\frac{1}{9} \sum_{n=3}^{\infty}(-1)^{n} \frac{y^{n}}{n !}$

From eq. (180A)

$b_{1}=1 \quad b_{2}=\frac{1}{2} \quad$ and for $n \geq 3 \quad b_{n}=\frac{1}{9} \frac{(-1)^{n}}{n !}$

Substituting eqs. (233) - (236) into eq. (182) yields

$\alpha=\frac{2}{3}$

Inserting eqs. (245) - (246) into eq. (184) yields

$a_{1}=1 \quad a_{2}=\frac{5}{6} \quad a_{3}=\frac{9}{3 ! 3^{3}}$

$a_{n}=\frac{(-1)^{n+1}}{n !}\left(\frac{2}{3} \sum_{m=1}^{n-1}(-1)^{m} m ! a_{m}-\frac{1}{9}\right)$ for $n \geq 3$

Proof (by induction) that $a_{n}=(-1)^{n+1} \frac{9}{n: 3^{n}}$ for $n \geq 3$ 


\section{Suppose}

$$
a_{n}=(-1)^{n+1} \frac{9}{n ! 3^{n}}
$$

Writing eq. (248) for $n+1$

$$
a_{n+1}=\frac{(-1)^{n}}{(n+1)}:\left(\frac{2}{3} \sum_{m=1}^{n-1}(-1)^{m} \cdot a_{m}+\frac{2}{3}(-1)^{n} n ! a_{n}-\frac{1}{9}\right)
$$

Dividing eq. (248) by $-3(n+1)$

$\frac{-a n}{3(n+1)}=\frac{(-1)^{n}}{3(n+1) !}\left(\frac{2}{3} \sum_{m=1}^{n-1}(-1)^{m} m: a_{m}-\frac{1}{9}\right)$

Subtracting eq. (251) from eq. (250)

$$
a_{n+1}+\frac{a_{n}}{3(n+1)}=\frac{(-1)^{n}}{3(n+1) !}\left(\frac{4}{3} \sum_{m=1}^{n-1}(-1)^{m} m ! a_{m}+(-1)^{n} 2 n ! a_{n}-\frac{2}{9}\right)
$$

Substituring $a_{n}$ from eq. (248) into eq. (252)

$a_{n+1}+\frac{a_{n}}{3(n+1)}=0$

Substituting eq. (249) into eq. (253)

$a_{n+1}=\frac{(-1)^{n+2} 9}{(n+1) ! 3^{n+1}}$

Eq. (249) is true for $n=3$; thus by eq. (254) the assertion is proven.

Thus by eq. (180)

$$
\begin{aligned}
& H_{2}(w, y)=w^{2}\left[y+\frac{5}{6} y^{2}+\sum_{n=3}^{\infty}(-1)^{n+1} \frac{9}{n ! 3^{n}} y^{n}\right] \\
& \operatorname{VAR}=H_{2}(w, y)-M_{1}^{2}(w, y)
\end{aligned}
$$


Using eqs. (241) and (255)

$$
\text { VAR }=w^{2}\left[y-\frac{1}{6} y^{2}+\sum_{n=3}^{\infty}(-1)^{n+1} \frac{9}{n ! 3^{n}} y^{n}\right]
$$


The exit kerne1s are biased so that absorption cannot occur and the event score generated by a particle of unit weight is $\sigma$ times the distance travelled between events.

$$
\begin{aligned}
& A_{0}\left(R^{\prime}\right)=0 \text { no absor,tion, "survival biased" } \\
& \begin{array}{ll}
T\left(R, R^{+}\right)=T^{*}\left(R, R^{+}\right) & w_{t}=1 \\
\sigma\left(R^{\prime}\right)=\sigma^{*}\left(R^{\prime}\right) & w_{U}=1
\end{array} \mid \begin{array}{l}
\text { next collision location } \\
\text { not biased }
\end{array} \\
& E\left(R^{\prime}, R^{\prime \prime}\right)=\frac{3}{2} E^{\star}\left(R^{\prime}, R^{\prime \prime}\right) \quad W_{\varepsilon_{1}}=\frac{2}{3} \quad A_{1}=\frac{3}{2} A_{1}^{*}=\frac{1}{2} \\
& \varepsilon_{2}\left(R^{\prime}, R_{1}, R_{2}\right)=\frac{3}{2} \varepsilon_{2}^{*}\left(R^{\prime}, R_{1}, R_{2}\right) \quad w_{\varepsilon_{2}}=\frac{2}{3} \quad A_{2}=\frac{3}{2} A_{2}^{*}=\frac{1}{2} \\
& P_{d}\left(P, P^{\prime}, s\right)=P_{k}\left(P^{\prime}, P^{\prime}, s\right)=P_{d}^{*}\left(P, P^{\prime}, s\right)=P_{k}^{*}\left(P, P^{\prime}, s\right) \\
& =\delta\left(s-w^{\prime} \sigma x^{\prime}\right)=\delta\left(s-w^{\prime} y^{\prime}\right) \quad k=0,1, ?
\end{aligned}
$$

Substituting eqs. (258) - (263) into eq. (177)

$$
\begin{aligned}
& Q_{1}=w y e^{-y}+w \int_{0}^{y} d y^{\prime} e^{-y^{\prime}} y^{\prime} \\
& Q_{1} / w=y e^{-y}+e^{-y}(-y-1)+1=1-e^{-y} \\
& Q_{1} / w=\sum_{n=1}^{\infty}(-1)^{n} \frac{y^{n}}{n !}
\end{aligned}
$$

Substituting eqs. (259) - (262) into eq. (182) for $r=1$

$$
\alpha=1
$$


$Q_{1}$ and $\alpha$ are the same as in game 1; thus following the same steps as in game 1 yields

$$
M_{1}(w, y)=w y
$$

Substituting eqs. (258) - (263) and (268) into eq. (178)

$$
\begin{aligned}
Q_{2} & =w^{2} y^{2} e^{-y}+w^{2} \int_{0}^{y} d y^{\prime} e^{-y^{\prime}} A_{1}\left\{y^{\prime 2}+2 y^{\prime} w_{\varepsilon_{1}}\left(y-y^{\prime}\right)\right\} \\
& +w^{2} \int_{0}^{y} d y^{\prime} e^{-y^{\prime}} A_{2}\left\{y^{\prime 2}+4 y^{\prime} w_{\varepsilon_{2}}\left(y-y^{\prime}\right)+2 w_{\varepsilon_{2}}^{2}\left(y-y^{\prime}\right)^{2}\right\}
\end{aligned}
$$

Using eqs. (259) - (262) and (A6) - (A7)

$$
Q_{2} / w^{2}=y^{2} e^{-y}-v\left[y^{2}\right]+2 y v[y]+\frac{4}{9} T\left[y^{2}\right]
$$

Usings Eqs. (A8.1) and (A11)

$Q_{2} / w^{2}=\frac{10}{9} e^{-y}-\frac{10}{9}+\frac{10}{9} y+\frac{4}{9} y^{2}$

$Q_{2} / w^{2}=y^{2}+\frac{10}{9} \sum_{n=3}^{\infty}(-1)^{n} \frac{y^{n}}{n !}$

Froin eqs. (180A) and (272)

$b_{1}=0 \quad b_{2}=1 \quad b_{n}=(-1)^{n} \frac{10}{9 n !}, n \geq 3$

Substituting eqs. (259) - (262) into eq. (182) for $r=2$

$\alpha=\frac{2}{3}$ 
Using eqs. (273) and (274) in eq. (184)

$$
\begin{aligned}
& a_{1}=0 \quad a_{2}=1 \quad a_{3}=\frac{6}{3 ! 3^{3}} \\
& a_{n}=\frac{(-i)^{n+1}}{n !}\left(\frac{2}{3} \sum_{m=1}^{n-1}(-1)^{m} m ! a_{m}-\frac{10}{9}\right) \quad n \geq 3
\end{aligned}
$$

Proof (by induction) that $a_{n}=(-1)^{n+1} \frac{6}{n ! 3^{n}}$ for $n \geq 3$

Suppose

$$
a_{n}=(-1)^{n+1} \frac{6}{n ! 3^{n}}
$$

Writing eq. (276) for $n+1$

$$
a_{n+1}=\frac{(-1)^{n}}{(n+1) !}\left(\frac{2}{3} \sum_{m=1}^{n-1}(-1)^{n ! n} m ! a_{m}+(-1)^{n} \quad \frac{2}{3} n ! a_{n}-\frac{10}{9}\right)
$$

Dividing eq. (276) by $-3(n+1)$

$\frac{-a_{n}}{3(n+1)}=\frac{(-1)^{n}}{3(n+1) !}\left(\frac{2}{3} \sum_{m=1}^{n-1}(-1)^{m_{m}} a_{m}-\frac{10}{9}\right)$

Subtracting eq. (278) from eq. (277)

$$
\begin{aligned}
a_{n+1} & +\frac{a_{n}}{3(n+1)} \\
= & \frac{(-1)^{n}}{3(n+1) !}\left(\frac{4}{3} \sum_{m=1}^{n-1}(-1)^{m} m ! a_{m}-\frac{2 \cdot 10}{9}+(-1)^{n} 2 n ! a_{n}\right)
\end{aligned}
$$

Using eq. (276) in the right hand side of eq. (279)

$$
a_{n+1}+\frac{a_{n}}{3(n+1)}=0
$$

Substituting eq. (276A) into eq. (280)

$$
a_{n+1}=(-1)^{n+2}-\frac{6}{(n+1) ! 3^{n+1}}
$$


Now eq. (276) is true for $n=3$, so by induction it is true for $n \geq 3$.

QED.

From eq. (180)

$M_{2}(w, y)=w^{2} y^{2}+w^{2} \sum_{n=3}^{\infty}(-1)^{n+1} \frac{6}{n ! 3^{n}} y^{n}$

(281)

Using eqs. (268) and (281)

VAR $=M_{2}\left(w_{0} y\right)-M_{1}^{2}(w, y)=w^{2} \sum_{n=3}^{\infty}(-1)^{n+1} \frac{5}{n ! 3^{n}} y^{n}$

(282)

86 
Comporisons of Four Estimators for Collisions

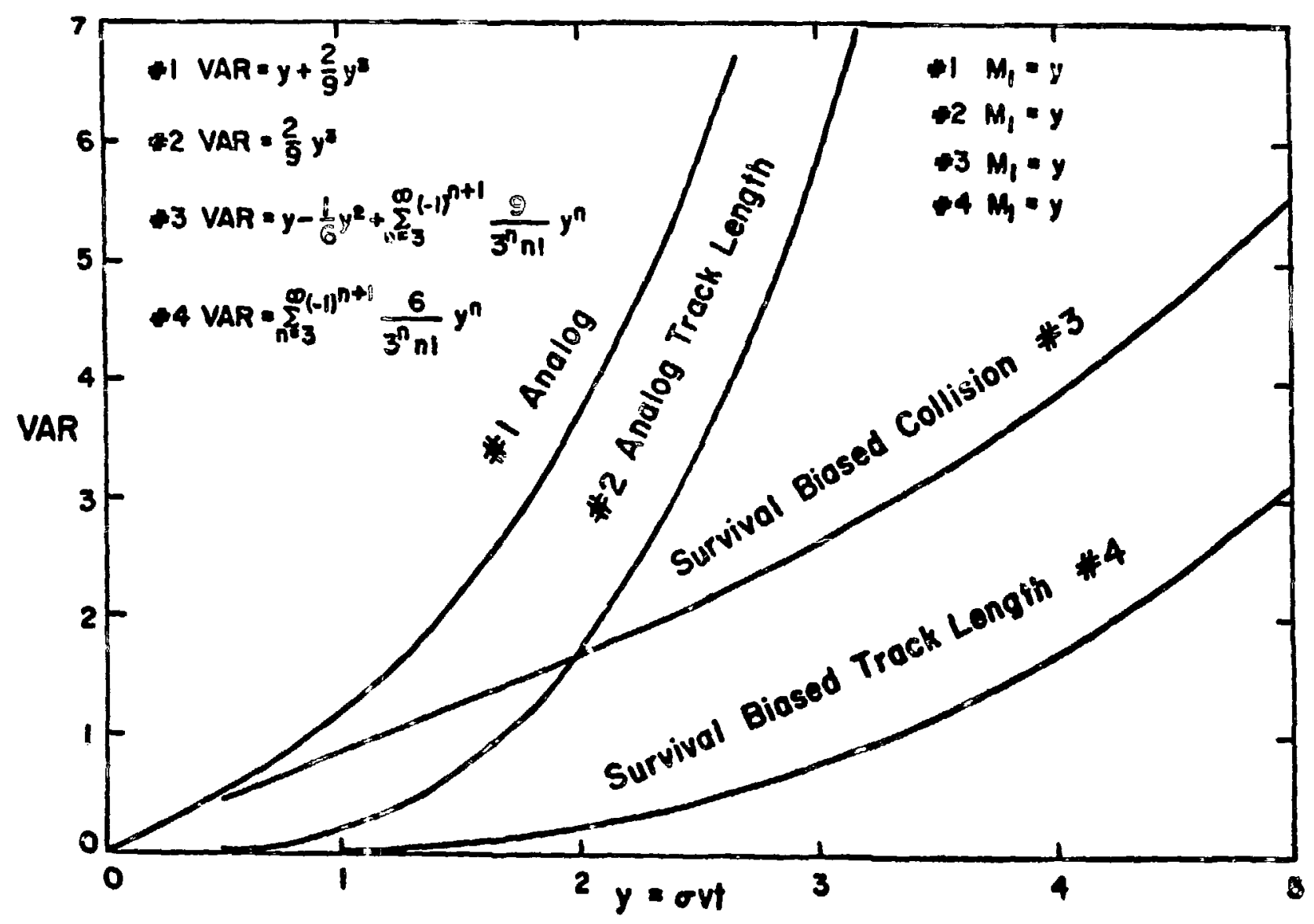




\section{Numerical Comparison of Four Estimators for Collisions}

Monte Carlo calculations were performed (using the MCNP code at the Los Alamos Scientific Laboratory) for each of the four games in IV-C. Forty thousand neutron histories were followed in each game. The sample means and variances are compared below with the theoretical means and variances predicted in IV-C.

$$
\begin{aligned}
& \bar{M}_{1}=\text { sample mean score } \\
& \bar{M}_{2}=\text { sample mean-square score } \\
& \frac{\text { VAR }}{=\bar{M}_{2}}-\bar{M}_{1}^{2}=\text { sample variance } \\
& \bar{S}_{m}=(\overline{V A R} / 40000)^{1 / 2}=\text { standard deviation of the sample mean } \\
& M_{1}=\text { mean score } \\
& M_{2}=\text { mean-square score } \\
& \text { VAR }=M_{2}-M_{1}^{2}=\text { variance } \\
& S_{m}=(V A R / 40000)^{1 / 2}=\text { standard deviation of the mean }
\end{aligned}
$$


Game I Analog Collision Estimator

$\begin{array}{lccccc} & y=1 & 2 & 3 & 4 & 5 \\ \bar{M}_{\mathrm{T}} & .99786 & 1.9914 & 3.0000 & 3.9954 & 4.9828 \\ \bar{M}_{2} & 2.2039 & 7.6530 & 17.9861 & 33.9382 & 57.7933 \\ \overline{\text { VAR }} & 1.2082 & 3.6875 & 8.9857 & 17.9748 & 32.3650 \\ \bar{S}_{\mathrm{m}} & .00550 & .00960 & .01499 & .02120 & .02845 \\ M_{\mathrm{m}} & 1.0000 & 2.0000 & 3.0000 & 4.0000 & 5.0000 \\ M_{2} & 3.2222 & 7.7778 & 18.0000 & 39.2222 & 57.7778 \\ \text { VAR } & 1.2222 & 3.7778 & 9.0000 & 18.2222 & 32.7778 \\ S_{m} & .00553 & .00972 & .01500 & .02 ? 34 & .02863\end{array}$


Game 2 Track Length Estimator

$\begin{array}{lccccc} & y=1 & 2 & 3 & 4 & 5 \\ \bar{M}_{1} & .99935 & 2.0009 & 3.0027 & 4.0002 & 4.9816 \\ \bar{M}_{2} & 1.2208 & 5.7783 & 15.0690 & 30.1939 & 52.3384 \\ \overline{\text { VAR }} & .22207 & 1.7745 & 6.0524 & 14.1815 & 27.5215 \\ \bar{S}_{m} & .00236 & .00666 & .01230 & .01883 & .02623 \\ M_{1} & 1.0000 & 2.0000 & 3.0000 & 4.0000 & 5.0000 \\ M_{2} & 1.2222 & 5.7778 & 15.0000 & 30.2222 & 52.7778 \\ \text { VAR } & .22222 & 1.7778 & 6.0000 & 14.2222 & 27.7778 \\ S_{m} & .00236 & .00667 & .01224 & .01886 & .02635\end{array}$


Game 3 Survival Biased Collision Estimator

$\begin{array}{lccccc} & y=1 & 2 & 3 & 4 & 5 \\ \bar{M}_{1} & .99735 & 2.0001 & 2.9976 & 3.9895 & 4.9831 \\ \bar{M}_{2} & 1.8763 & 5.7110 & 11.6566 & 19.8487 & 30.4348 \\ \overline{\text { VAR }} & .88156 & 1.7089 & 2.6711 & 3.9331 & 5.6034 \\ \bar{S}_{m} & .00469 & .00654 & .00817 & .00992 & .01184 \\ M_{1} & 1.0000 & 2.0000 & 3.0000 & 4.0000 & 5.0000 \\ M_{2} & 1.8846 & 5.7126 & 11.6891 & 19.9610 & 30.6335 \\ \text { VAR } & .88455 & 1.7126 & 2.6891 & 3.9610 & 5.6335 \\ S_{m} & .00470 & .00654 & .00820 & .00995 & .01187\end{array}$


Game 4 Survival Biased Track Length Estimator

$\begin{array}{lccccc} & y=1 & 2 & 3 & 4 & 5 \\ \bar{M}_{1} & .99928 & 1.9989 & 2.9962 & 3.9951 & 4.9862 \\ \bar{M}_{2} & 1.0328 & 4.2499 & 9.7701 & 17.7210 & 28.0534 \\ \overline{\text { VAR }} & .03420 & .25409 & .79303 & 1.7605 & 3.1910 \\ \bar{s}_{m} & .00092 & .00252 & .00445 & .00663 & .00893 \\ M_{1} & 1.0000 & 2.0000 & 3.0000 & 4.0000 & 5.0000 \\ M_{2} & 1.0341 & 4.2528 & 9.7927 & 17.7518 & 28.2001 \\ \text { VAR } & .03415 & .25283 & .79272 & 1.7518 & 3.2001 \\ S_{m} & .00092 & .00251 & .00445 & .00662 & .00894\end{array}$


Chapter V. Conclusion

The equations for $M_{1}$ and $M_{2}$ are useful for at least two reasons. First, it is possible to use the equations to prove certain well knowri or conjectured results ${ }^{2,3,4,7,8}$ (e.g. see IV-A of this work). Second, the equations are useful in predicting errors in specific Monte Carlo transport calculations (e.g. see IV-C). Often a proposed variance reduction technique will reduce the number of histories required, but increase the computation time per history. The equations for $\mathrm{H}_{\mathrm{q}}$ and $M_{2}$ allow prediction of how much a specific technique reduces the number of histories required, to be weighed against a change in the time requirel per history.

T'ie moment equations derived here are linear integral equations, and thus can be solved by several techniques. One possibility is simply to truncate the Neumann series of eq. (C7). Another possibility is to convert the moment equations to their integro-differential forms as Amster and Djomehri ${ }^{1}$ do, and then use a $P_{N}$ or an $S_{N}$ solution technique. (Sarkar and Prasad ${ }^{3}$ have used a $P_{1}$ approximation to the integro-differential forms of the moments equations to study the exponential transform.)

This work generalizes the theory developed by Amster and Djomehri ${ }^{1}$ to include:

1) Error prediction in Monte Carlo transport calculations of timedependent multiplying systems, even when supercritical. 
2) Score distributions which depend on the preceding event point as well as the current event point. This allows prediction of errors in calculations using a track length estimator.

3) Russian Roulette and splitting events. These are standard variance reducing techniques. 


\section{APPENDIX A - Useful Results}

\section{Result \#1}

Consider

$B=\int \psi\left(P, s-s^{*}, t\right) s^{r} d s$

Let $s=s-s^{*}$, that is $s=s+s^{*}$

$B=\int \psi(P, s, t)\left(s+s^{*}\right)^{r} d s$

Using the binomial theorem

$$
\begin{aligned}
B & =\int J s \sum_{n=0}^{r}\left(\begin{array}{l}
r \\
n
\end{array}\right) s^{\star^{r-n}} s^{n} \psi(P, \hat{s}, t) \\
& =\sum_{n=0}^{r}\left(\begin{array}{l}
r \\
n
\end{array}\right) s^{*}{ }^{r-n} \int d \hat{s} \psi(P, \hat{s}, \hat{\imath}) \hat{s}^{n}
\end{aligned}
$$

Using eq. (81)

$$
\beta=\sum_{n=0}^{r}\left(\begin{array}{l}
r \\
n
\end{array}\right) s^{*-n} M_{n}(P, t)=\int d s \psi\left(P, s-s^{*}, t\right) s^{r}
$$




\section{Resuli \#2}

Consider the operators

$T[R(y)]=\int_{0}^{y} e^{-y^{\prime}} R\left(y-y^{\prime}\right) d y^{\prime}$

$V[R(y)]=\int_{0}^{y} e^{-y^{\prime}} R\left(y^{\prime}\right) d y^{\prime}$

Froin integral tables 5

$$
\begin{array}{ll}
V\left[y^{m}\right]=m ! e^{-y} \sum_{j=m+1}^{\infty} \frac{y^{j}}{j !} & \text { for } m \geq 0 \\
V\left[y^{m}\right]=m !\left(1-e^{-y} \sum_{j=0}^{m} \frac{y^{j}}{j !}\right) & \text { for } m \geq 0
\end{array}
$$

Let $x=y-y^{\prime}$ in eq. (A6)

$T[R(y)]=e^{-y} \int_{0}^{y} e^{x} R(x) d x$

From integral tables 5

$$
\begin{aligned}
& T\left[y^{m}\right]=(-1)^{m+1} m ! \sum_{j=m+1}^{\infty}(-1)^{j} \frac{y^{j}}{j !} \quad \text { for } m \geq 0 \\
& T\left[y^{m}\right]=(-1)^{m+1} m:\left(e^{-y}-\sum_{j=0}^{m}(-1)^{j} \frac{y^{j}}{j !}\right) \quad \text { for } m \geq 0
\end{aligned}
$$


Result \#3

Consider the sum

$s=\sum_{b_{1}=0}^{1} \cdots \sum_{b_{k}=0}^{1} x^{b_{1}+\cdots+b_{k}}(1-x)^{k-b_{1} \cdots \cdots-b_{k}}$.

This sum can be rewritten

$S=\sum_{b_{1}=0}^{1} x^{b_{1}}(1-x)^{1-b_{1}} \ldots \sum_{b_{k}=0}^{1} x^{b_{k}}(1-x)^{1-b_{k}}$,

but

$\sum_{n=0}^{1} x^{n}(1-x)^{1-n}=1$

thus

$S=1$.

Consider the sum

$s=\sum_{b_{1}=0}^{1} \cdots \sum_{b_{k}=0}^{1} x^{b_{1}+\cdots+b_{k}}(1-x)^{k-b_{1} \cdots-\cdots-b_{k}} \sum_{j} F(j) \delta_{b_{j}, 1}$

Rearranging the sum over the b's as in eq. (A13) and using eq. (A14)

$S=\sum_{j} F(j) \sum_{b_{j}=0}^{l} x^{b}(1-x)^{1-b_{j}} \delta_{b_{j}, 1}$ 
or

$$
S=x \sum_{j} F(j)
$$

(A18)

Consider the sum

$$
\begin{aligned}
& s=\sum_{b_{l}=0}^{1} \cdots \sum_{b_{k}=0}^{l} x^{b_{l}+\cdots+b_{k}}(1-x)^{k-b_{1} \cdots \cdots-b_{k}} \sum_{j} F(j) \delta_{b_{j}, 1} \\
& x \sum_{\ell} G(\Omega) \delta_{b_{\ell}, 1}
\end{aligned}
$$

Rearranging the sum over the b's as in eq. (A13) and using eq. (A14)

$$
\begin{aligned}
& S=\sum_{j} F(j) \sum_{b_{j}=0}^{l} x^{b_{j}}(1-x)^{1-b_{j}} \delta_{b_{j}, l} \sum_{\ell} G(l) \\
& \therefore \sum_{b_{\ell}=0}^{1} x^{b_{l}}(1-x)^{1-b_{\ell}} \delta_{b_{\ell, l}} \text {, or } \\
& S=x^{2} \sum_{j} F(j) \sum_{\ell} G(l)
\end{aligned}
$$

(A20)

(A21)

98 


\section{Appendix B}

Simple Example of an Unreliable Sample Variance

Consider particles trying to penetrate a thick shield. If the shield is any good, very few of the particles will penetrate the shield. Thus, it is possible to simulate a huge number of particles without any of the particles penetrating the shield. If none of the simulated particles penetrate the shield, the sample mean and the sample variance will both be zero. However, even for the best shields the probability of penetration is some number $\varepsilon>0$. The sample mean is thus in error, at the same time the samp.e variance is indicating zero error. 
APPENDIX $C$

CONVERGENCE OF THE NEWMAN SERIES

FOR EQUATION (125)

Let $x^{\Delta}$ be the coordinate along $\vec{\Omega}$, the direction of the neutron's flight. Let

$$
\vec{r}^{\prime}-\vec{r}=\left(x^{\prime}, y^{\prime}, z^{\prime}\right)
$$

so that the neutron's position is described in a Cartesian coordinate system whose origin is at $\vec{r}$ and whose $X^{\prime}$ axis lies along $\vec{\Omega}$. Let

$$
\begin{aligned}
& x^{\prime}=v t^{\prime} \\
& \vec{r}^{\prime}=\vec{r}+\vec{\delta} x^{\prime}=\vec{r}+\vec{v} t^{\prime} \\
& T\left(R, R^{\prime}\right)=\delta\left(\vec{v}-\vec{v}^{\prime}\right) \delta\left(y^{\prime}\right) \delta\left(z^{\prime}\right) T\left(R, \vec{r}^{\prime}, \vec{v}^{\prime}\right) \\
& \sigma\left(R^{\prime}\right)=\sigma\left(\vec{r}^{\prime}, \vec{v}^{\prime}\right) \\
& \varepsilon_{k}\left(R^{\prime}, R_{1}, \cdots, R_{k}\right)=\varepsilon_{k}\left(\vec{r}^{\prime}, \vec{v}^{\prime}, R_{1}, \cdots, R_{k}\right)
\end{aligned}
$$

Thus eu. (125) may be rewritten:

$$
\begin{aligned}
& M_{r}(R, w, t)=\int_{0}^{t} v d t^{\prime} T\left(R, \vec{r}+\vec{v} t^{\prime}\right) \sigma\left(\vec{r}+\vec{v} t^{\prime}, \vec{v}\right) \\
& x \sum_{k=1}^{\infty} \int d R_{1} \cdots \int d R_{k} \varepsilon_{k}\left(\vec{r}+\vec{v} t^{\prime}, \vec{v}, R_{1}, \cdots, R_{k}\right)\left\{H\left(w w_{t} w_{\sigma} w_{\varepsilon_{k}}-w_{c o}\right)\right.
\end{aligned}
$$

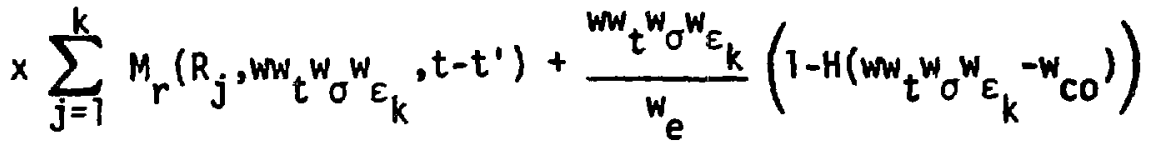

$$
\begin{aligned}
& \left.x \sum_{j=\{}^{k} M_{r}\left(R_{j}, w_{e}, t-t^{\prime}\right)\right\}+Q_{r}(R, w, t)
\end{aligned}
$$

100 
Define the operator

$$
\begin{aligned}
& x\left(t^{\prime}\right) G\left(R, w, t-t^{\prime}\right)=v T\left(R, \vec{r}+\vec{v} t^{\prime}\right) \sigma\left(\vec{r}+\vec{v} t^{\prime}, \vec{v}\right) \\
& x \sum_{k=1}^{\infty} \int d R_{1} \cdots \int d R_{k} \varepsilon_{k}\left(\vec{r}+\vec{v} t \cdot, \vec{v}, R_{1}, \cdots, R_{k}\right)\left\{H\left(w w_{t} w_{\sigma} w_{\varepsilon_{k}}{ }^{-w_{c o}}\right)\right.
\end{aligned}
$$

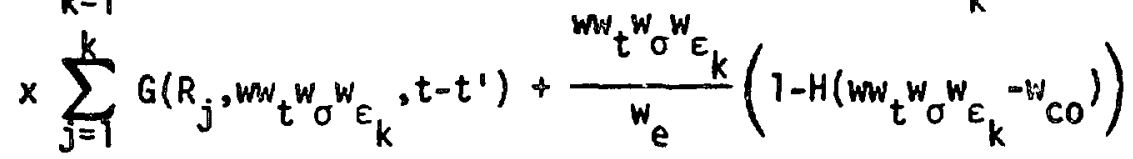

$$
\begin{aligned}
& \left.x \sum_{j=1}^{k} G\left(R_{j}, w_{e}, t-t^{\prime}\right)\right\}
\end{aligned}
$$

Thus

$M_{r}\left(R, w_{0} t\right)=\int_{0}^{t} d t_{1} X\left(t, M_{r}\left(R, w, t-t_{1}\right)+Q_{r}(R, w, t)\right.$

or replacing $t$ by $t-t^{\prime}$ and $t$, by $t^{\prime \prime}$

$M_{r}\left(R, w_{0} t^{-} t^{\prime}\right)=\int_{0}^{t-t^{\prime}} d t^{\prime \prime} \chi\left(t^{\prime \prime}\right) M_{r}\left(R, w, t-t^{\prime}-t^{\prime \prime}\right)+Q_{r}\left(R, w_{0}, t-t^{\prime}\right)$

Now use eqs. (C3)-(C4) to generate a Neumann series.

$$
\begin{aligned}
& M_{r}(R, w, \dot{c})=\int_{0}^{t} d t_{1} x\left(t_{1}\right)\left\{\int_{0}^{t-t_{1}} d t_{2} x\left(t_{2}\right) M_{r}\left(R, w, t-t_{1}-t_{2}\right)\right. \\
& \left.+Q_{r}\left(R, w, t-t_{1}\right)\right\}+Q_{r}(R, w, t)
\end{aligned}
$$

Let $\tau_{n}=t_{n}+t_{2}+\cdots+t_{n}$ and substitute eq. (C4) into eq. (C5) far the next term in the series

$$
\begin{aligned}
& M_{r}\left(R_{0}, w_{0} t\right)=Q_{v}(R, w, t)+\int_{0}^{t} d t_{1} x\left(t_{1}\right) Q_{r}\left(R, w, t-T_{7}\right) \\
& +\int_{0}^{t} d t_{1} x\left(t_{1}\right) \int_{0}^{t-T_{2}} d t_{2} x\left(t_{2}\right)\left\{\int_{0}^{t-T_{2}} d_{t_{3}} x\left(t_{3}\right) M_{r}\left(R, w, t-T_{3}\right) .\right. \\
& \left.+Q_{r}\left(R_{0} w, t-T_{2}\right)\right\}
\end{aligned}
$$


Repetitively substituting for $M_{r}\left(R, w, t-T_{n}\right)$ yields:

$i_{r}(R, w, t)=a_{r}(R, w, t)+\sum_{q=1}^{\infty} \int_{0}^{t} d t_{1} x\left(t_{1}\right) \int_{0}^{t-T} d t_{2} x\left(t_{2}\right) \ldots$

$\left.x \int_{0}^{t-T} q-T_{q} d t_{q}\right) Q_{r}(R, w, t-T q)$, the Meumann series.

Physically $T_{n}$ is the time elapsed between a particle's birth ("zero ${ }^{\text {th }}$ event") and $n^{\text {th }}$ event, $t_{n}$ is the elapsed time between a particle's $n-l^{\text {st }}$ and $n^{\text {th }}$ events, and finally, $t-T_{n}$ is the history time remaining after a particle's $n^{\text {th }}$ event.

In the following theorem, unless otherwise specified, eqs. (C8)(C15) hold for all possible arguments of the bounded function. All maxima are taken over the full range of all variables. For instance, $\max (A, B, C)=\max (A(X), B(Y), C(Z))$

$$
\text { all } x, y, Z
$$

where $X, Y, Z$ may be $n$-tuples of vectors.

Theorem $\mathrm{C}$. The Neumann series (C7) converges if there exists a uniform bound, $B>1$, such that:

1) The particle starting weight is bounded. $w<B$

2) All weight multipliers are bounded.

$$
w_{t}<B, w_{\sigma}<B \text {, and } w_{\varepsilon_{k}}<B
$$

3) The exit weight from a Russian Roulette game is bounded.

$$
w_{e}<B \text { and } w_{c o}\left(R^{\prime}\right)<w_{e}\left(R^{\prime}, R_{1}, \cdots, R_{k}\right)
$$


4) The speed is bounded.

$1<B$

5) The macroscopic total cross section is bounded, thus

$$
T\left(R, \vec{r}+\vec{v} t^{\prime}\right) \sigma\left(\vec{r}+\vec{v} t^{\prime}, \vec{v}\right)<B
$$

6) The number of particles departing an event is bounded. $k<B$

7) The source function $Q_{r}\left(R, w_{0}, t_{0}\right)$ is bounded

$$
\begin{aligned}
q_{r}\left(R, w_{0}, t_{0}\right)<B \quad \text { for } \quad 0 & \leq w_{0} \leq \max \left(w_{t} w_{\sigma} w_{\varepsilon_{k}}, w_{e}, w\right) \\
0 & \leq t_{0} \leq t
\end{aligned}
$$

Before proving this theorem, let us discuss conditions 1) -7 ). Conditions 1), 2), and the first part of 3) ensure that the particle weights are always finite. The second part of 3) simply says that in Russian Roulette, the exit weight is larger than the cutoff. The speed of light bounds any particle velocity, so condition 4) holds. Condition 5) is sactisfied for any real problems. (For delta function cross sections condition 5) could be altered to $\int_{t_{0}-\varepsilon}^{t_{0}+\varepsilon} T\left(R, \vec{r}+\vec{v} t^{\prime}\right) \sigma\left(\vec{r}+\vec{v} t^{\prime}, \vec{v}\right) v d t^{\prime}<2 \varepsilon B v$, so that the expected number of next collisions in any time interval is bounded.) Condition 6) should always hold in practice, although it is somewhat stronger than the condition actually required:

$\sum_{k=1}^{\infty} k A_{k}<B$ 
That is, the mean number of particles departing an event is bounded.

The source term $Q_{r}(R, w, t)$ must be bounded because it is the first term of the Neumann series. (The limitation on $w_{0}$ in eq. (C14) is required since we desire to bound both $Q_{r}\left(R, w_{t} w_{0} w_{E_{k}}, t-t^{\prime}\right)$ and $Q_{r}\left(R, w_{e}, t-t^{\prime}\right)$ in order to say $x\left(t^{\prime}\right) Q_{r}\left(R, w, t-t^{\prime}\right)$ is bounded. $Q_{r}$ will be bounded if conditions 1) - 6) are met, $M_{\ell}$ is bounded for $\ell<r$, and the moments of the event score are bounded, f.e..

$\int d s p\left(P, P^{\prime}, s\right) s^{\ell}<s_{\max }$, for $\ell \leq r$.

For example, see eqs. (127) and (130).

Thus we proceed in the following manner. $Q_{1}$ is bounded and thus by theorem $C, M_{1}$ is bounded. But $M_{1}$ bounded implies $Q_{2}$ is bounded, and thus by theorem $C, M_{2}$ is bounded. But $M_{1}$ and $M_{2}$ bounded imply $Q_{3}$ is bounded, and so forth.

Proof of Theorem $C$

Consider a bounded function

$$
G\left(R, w_{0}, t_{0}\right)<G_{m} \quad \text { for } \quad \begin{aligned}
& 0 \leq t_{0} \leq t \\
& G_{m}>1
\end{aligned}
$$

Note that $H\left(W_{t} W_{\sigma}{ }^{W} \varepsilon_{k}{ }^{-W} c 0\right) \leq 1$ and that, since by assumption 3)

$w_{c o} \leq w_{e}$.

$\frac{{ }^{w}{ }_{t}{ }^{W} \sigma{ }^{W} \varepsilon_{k}}{w_{e}}\left(1-H\left(w_{t} w_{\sigma}{ }^{w} \varepsilon_{k}-W_{c o}\right)\right) \leq 1$ 
Using these facts and eq. (C2)

$$
\begin{aligned}
& x\left(t^{\prime}\right) G\left(R, w, t-t^{\prime}\right)<v T\left(R, \vec{r}+\vec{v} t^{\prime}\right) \sigma\left(\vec{r}+\vec{v} t^{\prime}, \vec{v}\right) \\
& x \sum_{k=1}^{\infty} \int d R_{1} \cdots \int d R_{k} \varepsilon_{k}\left(\vec{r}+\vec{v} t^{\prime}, \vec{v}, R_{1}, \cdots, R_{k}\right) 2 k G_{m^{\prime}}
\end{aligned}
$$

where the $2 k$ comes from the two sums on $j$ of eq. (C2). By eqs. (82), (C11), and (C12)

$x\left(t^{\prime}\right) G\left(R, w, t-t^{\prime}\right)<2 B^{2} G_{m} \sum_{k=1}^{\infty} k A_{k}\left(\vec{r}+\vec{v} t^{\prime}, \vec{v}\right)$.

At this point we insist

$\sum_{k=1}^{\infty} k A_{k}\left(\vec{r}+\vec{v} t^{\prime}, \vec{v}\right)<B_{1}$, where $B_{1}>1$,

which will certainly be true if $k$ is boinded, in view of eq. (83).

Thus

$$
x\left(t^{\prime}\right) G\left(R, w, t-t^{\prime}\right)<2 B^{2} B, G_{m}=C
$$

Now let's look at the $q^{\text {th }}$ term of the Neumann series of eq. (C7).

$$
\left.N_{q}=\int_{0}^{t} d t_{1} x\left(t_{1}\right) \int_{0}^{t-T} d t_{2} x\left(t_{2}\right) \cdots \int_{0}^{t-T} d t_{q}^{q} x l_{t_{q}}\right) Q_{r}\left(R, w, t-T_{q}\right)
$$

Since the functions $Q_{r}$ and 1 both meet conditions (C15) then by (C17)

$$
N_{q}<c^{q} \int_{0}^{t} d t \int_{0}^{t-T} d t_{2} \cdots \int_{0}^{t-T} d t_{q}^{q-1}=c^{q} \tau
$$




$$
\begin{aligned}
& \text { Recal1 that } T_{n}=t_{1}+\cdots+t_{n} \text { and let } \\
& y_{n}=t-T_{n}=t-T_{n-1}-t_{n} \text {, then } \\
& \tau=\int_{0}^{t} d y_{1} \int_{0}^{y_{1}} d y_{2} \int_{0}^{y_{2}} d y_{3} \cdots \int_{0}^{y} q-1 d y_{q} \\
& \tau=\int_{0}^{t} d y_{1} \cdots \int_{0}^{y_{q-3} d y_{q-2} \int_{0}^{y} q-2 y_{q-1} d y_{q-1}} \\
& \tau=\int_{0}^{t} d y_{1} \cdots \int_{0}^{y_{q-4} d y_{q-3}} \int_{0}^{y} q-3 \frac{1}{2 !} y_{q-2}^{2} q y_{q-2} \\
& \tau=\int_{0}^{t} \frac{1}{(q-1) !} y_{1}^{q-1} d y_{1}=\frac{t^{q}}{q !}
\end{aligned}
$$

Hence the $q^{\text {th }}$ term of the Neumann series is

$$
N_{q}<c^{q} t^{q} / q !
$$

Thus the Neumann series is less than the series for $\exp (C t)$ and hence Neumann series (C7) converges. This completes the proof of theorem $C$. 


\section{APPENDIX D}

\section{Approximation of a Bounded n-Dimensional Region \\ by Convex Subregions}

Theorem Any bounded n-dimensional set possessing a volume as defined by the Peano-Jordan content, can be approximated arbitrarily closely (in volume) by a finite number of closed convex, $n$-dimensional intervals which are separate.

This theorem can be demonstrated using well-known results from the theory of volume and measure in mathematics. Here the term closed interval is defined as the Cartesian product of $n$ one-dimensional closed intervals -- namely, a closed interval I is defined as the set of points $P=\left(x_{1}, x_{2}, \cdots, x_{n}\right)$ in $n$-space satisfying the inequalities
$a_{i} \leq x_{i} \leq b_{i}$
$1 \leq i \leq n$.

By separate it is meant that any two of the closed intervals have only boundary points in common.

The theorem follows immediately from the development of the theory of content. 9 A bounded set has content if the inner content is equal to the outer content, and each of these latter two quantities is approximated to any desired degree of accuracy by the volume of a finite sum of separate intervals. (The volume of an $n$-dimensional interval I is given by the product $\prod_{i=1}^{n}\left(b_{i}-a_{i}\right)$, as expected, ) Since an interval 
is convex, the result follows. Clearly the approximation is not unique in fack, there are infinitely many ways to make the approximation.

The above resuit states that whenever a bounded set in $n$-space has a volume as defined by the content, then the approximation by convex intervals exists. This definition of volume, al though superseded by the definition of measure (Lebesque) for purely mathematical reasons in the modern theory of integration, is entirely adequate for all practical applications. For the latter, any region occurring in a real problem will have the same volume measured by the two definitions, content and measure. It is true that unbounded regions can arise where the above result does not hold, but usually in practice unbounded regions either can be approximated by a finite set of convex subregions or else the unbounded region can bo pen' laced. wi theut any appreciable loss of accuracy, by a bounded region. 


\section{Acknowledgments}

I wish to thank Dr. Harvey Amster for the initial suggestion and early guidance of this thesis, and Dr. Edmond Cashwell (Los Alamos Scientific Laboratory) for his guidance in the later stages of this thesis. Dr. Cashwell's willingness to take over direction of this thes is in mid-course averted many potential problems. I also wish to thank the menbers of the thesis committee who read this thesis. In particular, Prof. Lawrence Grossman read this thesis carefully enough to make some very worthwhile suggestions.

The Department of Nuclear Engineering and the Los Alamos Scientific Laboratory (LASL) cooperated to ensure minimum disruption of my thes is after Dr. Amster left the University. Prof. Stanley Prussin and Mr. Paul whalen (LASL) were particularly helpful in arranging for me to complete the thesis under Dr. Cashwell, in the Monte Carlo group of the Theoretical Design Division. Division leader Dr. Raymond Pollock and group leader Dr. Thomas Hirons also deserve thanks for approving the arrangement. 


\section{References}

1H. J. AMSTER and M. J. DJOMEHRI, Hucl. Sci. Eng., 60, 131 (1976).

2. E. BOOTH and J. J. AMSTER, Nucl. Sci. Eng., 65, 273 (1978).

3P. K. SARKAR and M. A. PRASAD, unpublished results.

${ }^{4}$ I. LUX, Nucl. Sci. Eng., 67, 317 (1978).

${ }^{5}$ CRC Standard Mathematical Tables, Twenty-Third Edition, CRC Press.

65. GLASSTONE and M. C. EDLUND, The Elements of Nuclear Reactor Theory, D. Van Nostrand, Inc., New York (1952).

7. LuX, Nucl. Sci. Eng., 67, 107 (1978).

8. LuX, Nucl. Sci. Eng., 66, 258 (1978).

9. W. ROGOSINSKI, Volume and Integral, Interscience Publishers, Inc., New York (1952). 